\title{
Large-eddy Simulation of Turbulent Flows in A Heated Streamwise Rotating Channel
}

A Thesis Submitted to the Faculty of Graduate Studies of the University of Manitoba

in Partial Fulfilment of the Requirements for the Degree of

\author{
Master of Science
}

Department of Mechanical and Manufacturing Engineering University of Manitoba

Winnipeg, Manitoba

by

Ye Zhang

(C) Copyright Ye Zhang, March 2012. All rights reserved. 


\section{Abstract}

In this thesis, large-eddy simulation has been performed to investigate a heated plane channel flow subjected to streamwise system rotations. A variety of rotation numbers ranging from $R o_{\tau}=0$ to 15 have been tested in conjunction with two fixed low Reynolds numbers $R e_{\tau}=150$ and 300. The fundamental characteristics of the resolved velocity and temperature fields in terms of their mean and root-mean-square (RMS) values are investigated. Advanced physical features in terms of the transport of turbulent stresses, turbulent kinetic energy (TKE), heat fluxes and forward and backward scatter of local kinetic energy (KE) fluxes between the resolved and subgrid scales are also studied. Numerical simulations were performed using the conventional dynamic model (DM) and an advanced dynamic nonlinear model (DNM) for closure of the filter momentum equation, and an advanced dynamic full linear tensor thermal diffusivity model (DFLTDM) for closure of the filtered thermal energy equation. 


\section{Acknowledgements}

First of all, I would like to thank my supervisor, Professor Bing-Chen Wang, for accepting me as a Master student in his group, for his useful advice during my work, and also for his generous help in both academic and non-academic areas which have greatly encouraged me during my study and stay in Canada. My sincere thanks also go to Professor M. F. Tachie, Professor S. J. Ormiston and Professor R. W. Derksen for their advice and help during my course studies.

Over the past two years, I have consulted with many research fellows and students. I would like to specially thank Dr. Mamun Molla for many constructive suggestions. I would also like to thank Afzal Hossain Rupak, QianQiu Xun, Mohammad Saeedi and Iman Fatemi for all the academic discussion and happy time working with them.

I sincerely thank my friends X. H. Jiang, B. H. Feng, A. Tharku, C. Y. Guo, X. Y. Chen, Y. Zhang, H. T. Zhao, Y. C. Zhao, Y. M. Sun, D. Shi, Z. X. Li, Z. Y. Liang, Y. F. Yang and Y. T. Luo. I am grateful to all the friends I have made in Winnipeg for bringing me so many joyful moments and encouragement.

Finally, I would like to express my deep gratitude to my parents for their consistent and unconditional love and support. 
Dedicated to my loving parents and sister 


\section{Table of Contents}

Abstract

$\begin{array}{ll}\text { Acknowledgements } & \text { ii }\end{array}$

$\begin{array}{ll}\text { Dedication } & \text { iii }\end{array}$

Table of Contents $\quad$ iv

List of Tables vii

List of Figures viii

Nomenclature xii

1 Introduction 1

1.1 Turbulence: Basic Concepts and Research . . . . . . . . . . . . . . . 1

1.2 Characteristic Scales of Turbulence . . . . . . . . . . . . . . . . 3

1.3 Numerical Simulation of Turbulence . . . . . . . . . . . . . . . . . . 4

1.4 Subgrid-Scale Models in LES . . . . . . . . . . . . . . . . . . . . . 6

1.4.1 Dynamic Modelling for SGS Stresses . . . . . . . . . . . 6

1.4.2 Dynamic Modelling for SGS Heat Fluxes . . . . . . . . . . . 7

1.5 Motivation . . . . . . . . . . . . . . . . . . 9

1.6 Objectives of the Thesis . . . . . . . . . . . . . 13 
2.1 Filtering Operation . . . . . . . . . . . . . . 16

2.1 .1 Basic Concepts . . . . . . . . . . . . . 16

2.1.2 Discrete Filtering Process . . . . . . . . . . . . . 17

2.2 Filtered Governing Equations _... . . . . . . . . . . . . . 18

2.3 SGS Stress Models . . . . . . . . . . . . . . . . . . . . . 20

2.3.1 SGS Stress Model $1(\mathrm{DM}) \ldots \ldots 20$

2.3.2 SGS Stress Model $2(\mathrm{DNM}) \ldots \ldots . \ldots 23$

2.4 SGS Heat Flux Models . . . . . . . . . . . . . . . 25

2.4.1 SGS Heat Flux Model $1(\mathrm{DEDM}) \ldots \ldots . \ldots 25$

2.4.2 SGS Heat Flux Model 2 (DFLTDM) . . . . . . . . . . 26

2.5 Numerical Algorithm . . . . . . . . . . . . . . . . . . 27

2.6 Test Case and Computational Domain . . . . . . . . . . . . . . . . 31

2.7 Boundary Conditions . . . . . . . . . . . . . . . . . . . . . . . 32

3 Statistics of the Turbulent Thermal Flow Fields 33

3.1 Resolved Mean Velocity and Temperature . . . . . . . . . . . 33

3.2 Resolved Velocity and Temperature Fluctuations . . . . . . . . . . . . 41

3.3 Visualization of the Flow Field . . . . . . . . . . . . . . . . . . 44

4 Rotation Effects on Transport of Turbulent Quantities 46

4.1 Transport of Resolved Turbulent Stresses and TKE . . . . . . . . . . 46

4.1.1 Rotation Effect on the Resolved Turbulent Stresses . . . . . . 46

4.1.2 Rotation Effect on the Resolved TKE . . . . . . . . . 51 
4.2 Budgets of Shear Stresses and Heat Fluxes _ . . . . . . . . . . . 53

4.2.1 Budget of Shear Stresses _. . . . . . . . . . . . . 53

4.2 .2 Budget of Heat Fluxes . . . . . . . . . . . . . . . . . . 57

4.3 Transfer of Local KE between the Resolved and Subgrid Scale Motions 63

5 Conclusions and Reommendations for Future Work 67

5.1 Major Conclusions . . . . . . . . . . . . . . 67

5.2 Future Work . . . . . . . . . . . . . . . . . . . . . 69

$\begin{array}{ll}\text { References } & 71\end{array}$

$\begin{array}{lr}\text { Appendix } & 79\end{array}$

A Transport Equations for Resolved Turbulent Stresses

80 


\section{List of Tables}

1.1 Turbulence scales . . . . . . . . . . . . . . . . . . . . . 4

4.1 Production terms due to the mean turbulent shear $\left(P_{i j}\right)$ and rotation $\left(G_{i j}\right)$ stresses for a fully-developed rotating plane channel flow. . . . . 47 


\section{List of Figures}

2.1 A two-dimensional sketch of a typical control volume for a co-llocated grid system. . . . . . . . . . . . . . . . .

2.2 Computational domain for a heated streamwise rotating channel flow. The two Coriolis forces are: $-2 \Omega \bar{u}_{2}$ and $2 \Omega \bar{u}_{3} \ldots \ldots . \ldots$

3.1 Profiles of the resolved mean streamwise velocity for $R o_{\tau}=0$ and 15.0 at $R e_{\tau}=150, R o_{\tau}=0$ and 2.5 at $R e_{\tau}=300$ (predicted in conjunction with the DFLTDM). . . . . . . . . . . .

3.2 Profiles of the resolved mean spanwise velocity for $R o_{\tau}=0$ and 15.0 at $R e_{\tau}=150, R o_{\tau}=0$ and 2.5 at $R e_{\tau}=300$ (predicted in conjunction with the DFLTDM). . . . . . . . . . . . . .

3.3 Profiles of the resolved mean temperature for $R o_{\tau}=0$ and 15.0 at $R e_{\tau}=150, R o_{\tau}=2.5$ at $R e_{\tau}=300$ (predicted in conjunction with the DFLTDM). . . . . . . . . . . . . . . .

3.4 Profiles of the resolved mean streamwise velocity at various rotation numbers (predicted using the DNM and DFLTDM). . . . . . . .

3.5 Profiles of the resolved mean spanwise velocity at various rotation numbers (predicted using the DNM and DFLTDM). . . . . . . . .

3.6 Profiles of the resolved mean temperature at various rotation numbers (predicted using the DNM and DFLTDM). . . . . . . . . . . . 
3.7 Profiles of the resolved mean streamwise velocity for $R o_{\tau}=0$ and 15.0 at $R e_{\tau}=150, R o_{\tau}=0$ and 2.5 at $R e_{\tau}=300$, displayed using wall coordinates (predicted in conjunction with the DFLTDM). . . . . . .

3.8 Profiles of the resolved mean temperature for $R o_{\tau}=0$ and 15.0 at $R e_{\tau}=150, R o_{\tau}=2.5$ at $R e_{\tau}=300$, displayed using wall coordinates (predicted in conjunction with the DFLTDM). . . . . . . . .

3.9 Profiles of the resolved mean streamwise velocity at various rotation numbers, displayed using wall coordinates (predicted using the DNM

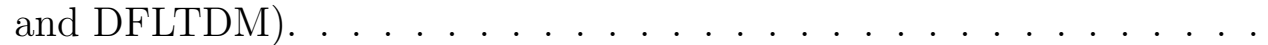

3.10 Profiles of the resolved mean temperature at various rotation numbers, displayed using wall coordinates (predicted using the DNM and

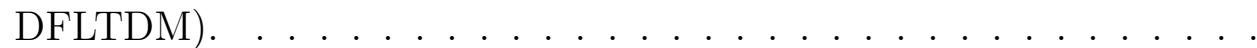

3.11 Resolved velocity fluctuations (predicted in conjunction with the DFLTDM). 42

3.12 Resolved temperature fluctuations for $R o_{\tau}=15.0$ at $R e_{\tau}=150, R o_{\tau}=$ 2.5 at $R e_{\tau}=300$ (predicted in conjunction with the DFLTDM). . . .

3.13 Resolved temperature fluctuations at different rotation numbers (predicted using the DNM and DFLTDM). . . . . . . . . .

3.14 Iso-surface of the wall-normal velocity field for $R e_{\tau}=300$ and $R o_{\tau}=15.45$

3.15 Contour of the streamwise vorticity field in the $\left(x_{1}, x_{3}\right)$-plane $\left(x_{2} / \delta=\right.$ 1.8, near the wall) for $R e_{\tau}=300$ and $R o_{\tau}=15$.

4.1 Resolved streamwise velocity fluctuations at various rotation numbers (predicted using the DNM and DFLTDM) . . . . . . . . .

4.2 Resolved wall-normal velocity fluctuations at various rotation numbers (predicted using the DNM and DFLTDM) . . . . . . . . . . . .

4.3 Resolved spanwise velocity fluctuations at various rotation numbers (predicted using the DNM and DFLTDM). . . . . . . . . . 
4.4 Resolved Reynolds shear stress $\left\langle\bar{u}_{1}^{\prime \prime} \bar{u}_{2}^{\prime \prime}\right\rangle^{+}$at various rotation numbers (predicted using the DNM and DFLTDM) . . . . . . . . . .

4.5 Resolved Reynolds shear stress $\left\langle\bar{u}_{2}^{\prime \prime} \bar{u}_{3}^{\prime \prime}\right\rangle^{+}$at various rotation numbers (predicted using the DNM and DFLTDM) . . . . . . . . .

4.6 Resolved Reynolds shear stress $\left\langle\bar{u}_{1}^{\prime \prime} \bar{u}_{3}^{\prime \prime}\right\rangle^{+}$at various rotation numbers (predicted using the DNM and DFLTDM). . . . . . . . . .

4.7 Resolved turbulent kinetic energy at various rotation numbers (nondimensionalized by $\tau_{w}^{a} / \rho$, predicted using the DNM and DFLTDM). .

4.8 Budget of shear stresses for $R o_{\tau}=15.0$ at $R e_{\tau}=150$ (non-dimensionalized using $\tau_{w}^{a} / \rho$, predicted in conjunction with the DFLTDM). Location of the vertical line along which the instantaneous SGS shear stress component is obtained: $x_{1} / L_{1} \approx 0.5$ and $x_{3} / L_{3} \approx 0.5 \ldots \ldots$. . . .

4.9 Budget of shear stresses for $R o_{\tau}=2.5$ at $R e_{\tau}=300$ (non-dimensionalized using $\tau_{w}^{a} / \rho$, predicted in conjunction with the DFLTDM). Location of the vertical line along which the instantaneous SGS shear stress component is obtained: $x_{1} / L_{1} \approx 0.5$ and $x_{3} / L_{3} \approx 0.5 \ldots \ldots$

4.10 Predicted Reynolds shear stress for $R o_{\tau}=0$ and 15 at $R e_{\tau}=150$ (non-dimensionalized using $\tau_{w}^{a} / \rho$, predicted in conjunction with the DFLTDM). Location of the vertical line along which the instantaneous SGS shear stress component is obtained: $x_{1} / L_{1} \approx 0.5$ and $x_{3} / L_{3} \approx 0.5 . \quad 55$

4.11 Predicted Reynolds shear stress at $R o_{\tau}=0$ and 2.5 for $R e_{\tau}=300$ (non-dimensionalized using $\tau_{w}^{a} / \rho$, predicted in conjunction with the DFLTDM). Location of the vertical line along which the instantaneous SGS shear stress component is obtained: $x_{1} / L_{1} \approx 0.5$ and $x_{3} / L_{3} \approx 0.5 . \quad 55$

4.12 Budget of heat fluxes in the wall-normal direction for $R o_{\tau}=15.0$ at $R e_{\tau}=150$ (non-dimensionalized using $q_{w h} / \rho c_{P}$ ). Location of the vertical line along which the instantaneous SGS wall-normal HF component is obtained: $x_{1} / L_{1} \approx 0.5$ and $x_{3} / L_{3} \approx 0.5$ 
4.13 Budget of heat fluxes in the wall-normal direction for $R o_{\tau}=2.5$ at $R e_{\tau}=300$ (non-dimensionalized using $q_{w h} / \rho c_{P}$ ). Location of the vertical line along which the instantaneous SGS wall-normal HF component is obtained: $x_{1} / L_{1} \approx 0.5$ and $x_{3} / L_{3} \approx 0.5 \ldots \ldots$

4.14 Total turbulent heat fluxes at $R o_{\tau}=15.0$ for $R e_{\tau}=150$ (predicted in conjunction with the DFLTDM).

4.15 Total turbulent heat fluxes at $R o_{\tau}=2.5$ for $R e_{\tau}=300$ (predicted in conjunction with the DFLTDM). . . . . . . . . . . . .

4.16 Total turbulent heat fluxes at various rotation number in streamwise direction (predicted in conjunction with the DFLTDM). . . . . .

4.17 Total turbulent heat fluxes at various rotation number in wall-normal direction (predicted in conjunction with the DFLTDM). . . . . . .

4.18 Total turbulent heat fluxes at various rotation number in spanwise direction (predicted in conjunction with the DFLTDM). . . . . .

4.19 Time- and plane-averaged SGS KE dissipation rates (non-dimensionalized using $\left(u_{\tau}^{a}\right)^{4} / \nu$, predicted using DNM in conjunction with the DFLTDM). Forward scatter: $\left\langle P_{r}^{+}\right\rangle$; backward scatter: $\left\langle P_{r}^{-}\right\rangle$; total SGS KE dissipation rate $\left\langle P_{r}\right\rangle \ldots \ldots \ldots \ldots \ldots \ldots$

4.20 Time- and plane-averaged SGS KE dissipation rates (non-dimensionalized using $\left(u_{\tau}^{a}\right)^{4} / \nu$, predicted using DM in conjunction with the DFLTDM). Forward scatter: $\left\langle P_{r}^{+}\right\rangle$; backward scatter: $\left\langle P_{r}^{-}\right\rangle$; total SGS KE dissipation rate $\left\langle P_{r}\right\rangle \ldots \ldots \ldots \ldots \ldots \ldots \ldots$ 


\section{Nomenclature}

\section{English Symbols}

$\begin{array}{ll}a & \text { coefficient for the pressure correction equation } \\ a_{j} & \text { test-grid level base vectors } \\ a_{l}, a_{m}, a_{n} & \text { filter coefficients } \\ A_{i} & \text { cross-sectional area of a control volume perpendicular to the } \\ & \text { i-th dimension } \\ \bar{A}_{i j} & \text { resolved velocity gradient tensor: } \partial \bar{u}_{i} / \partial x_{j} \\ b & \text { source term of the pressure correction equation } \\ b_{j} & \text { grid level base vectors } \\ c_{P} & \text { specific heat at constant pressure } \\ C & \text { constant } \\ C_{S}, C_{W}, C_{N} & \text { SGS stress model coefficients } \\ C_{\theta P}, C_{\theta G} & \text { SGS heat flux model coefficients } \\ D & \text { distance from the wall to the maximum streamwise velocity location } \\ D_{i j} & \text { tensor diffusivity } \\ e_{i j} & \text { residual tensor } \\ E & \text { norm of the residual tensor: } e_{i j} e_{i j} \\ \mathcal{E} & \text { norm of the residual vector: } \mathcal{E}_{j} \mathcal{E}_{j} \\ \mathcal{E}_{j} & \text { residual vector } \\ f & \text { function of space } \\ \mathcal{F}_{i} & \text { Coriolis force term } \\ & \end{array}$




\begin{tabular}{|c|c|}
\hline $\mathcal{H}_{i}$ & nonlinear term \\
\hline$H_{j}$ & test-grid level SGS heat flux vector \\
\hline$k$ & $\begin{array}{l}\text { index: } k=1,2 \text { and } 3 \text {; or wavenumber in the Fourier space; } \\
\text { or turbulent kinetic energy }\end{array}$ \\
\hline$k_{c}$ & cutoff wavenumber \\
\hline$i$ & index: $i=1,2$ and 3 \\
\hline$I, J, K$ & index numbers \\
\hline$j$ & index: $j=1,2$ and 3 \\
\hline$l$ & length scale for energy containing scale \\
\hline$L$ & length scale of the flow \\
\hline$L_{1}, L_{2}, L_{3}$ & channel length, height and width, respectively \\
\hline $\mathcal{L}_{i j}$ & resolved Leonard-type stress: ${\widetilde{\overline{u_{i}}}}_{j}-\tilde{\bar{u}}_{i} \tilde{\bar{u}}_{j}$ \\
\hline $\mathcal{L}_{j}$ & resolved vector: $\widetilde{\bar{u}_{j} \bar{\theta}}-\tilde{\bar{u}}_{j} \tilde{\bar{\theta}}$ \\
\hline$M_{i j}$ & differential tensorial function: $\alpha_{i j}-\tilde{\beta}_{i j}$ \\
\hline$N$ & number of the nodes for a 1-D discrete filter stencil \\
\hline$N_{i j}$ & differential tensorial function: $\zeta_{i j}-\tilde{\eta}_{i j}$ \\
\hline$N u$ & Nusselt number: $2 q_{w} /\left[\lambda\left(\theta_{D}-\theta_{w}\right) / D\right]$ \\
\hline$p$ & pressure \\
\hline$p_{e f f}$ & effective pressure \\
\hline$P_{i j}, G_{i j}$ & production terms for the resolved turbulent stresses \\
\hline$P_{j}, G_{j}$ & differential vector: $a_{j}-\tilde{b}_{j}$ \\
\hline$P_{q^{2}}, G_{q^{2}}$ & production terms for the resolved TKE \\
\hline$P_{r}$ & SGS KE dissipation rate: $-\tau_{i j} \bar{S}_{i j}$ \\
\hline$p^{\prime}$ & pressure correction \\
\hline$q_{w}$ & wall heat flux \\
\hline$q^{2}$ & resolved TKE: $\left(\left\langle\bar{u}_{1}^{\prime \prime 2}\right\rangle+\left\langle\bar{u}_{2}^{\prime \prime 2}\right\rangle+\left\langle\bar{u}_{3}^{\prime \prime 2}\right\rangle\right) / 2$ \\
\hline$R e$ & Reynolds number: $U L / \nu$ \\
\hline$R e_{\tau}$ & Reynolds number: $u_{\tau} \delta / \nu$ \\
\hline$R o_{\tau}$ & rotation number: $2 \Omega \delta / u_{\tau}$ \\
\hline $\bar{S}_{i j}$ & resolved strain rate tensor: $\left(\partial \bar{u}_{i} / \partial x_{j}+\partial \bar{u}_{j} / \partial x_{i}\right) / 2$ \\
\hline
\end{tabular}




$\begin{array}{ll}|\bar{S}| & \text { norm of } \bar{S}_{i j}:\left(2 \bar{S}_{i j} \bar{S}_{i j}\right)^{1 / 2} \\ t & \text { time } \\ \Delta t & \text { time step } \\ T_{i j} & \text { test-grid level SGS stress } \\ u_{i} & \text { velocity components: } i=1,2,3 \\ u_{\tau} & \text { wall friction velocity: } \sqrt{\tau_{w} / \rho} \\ u_{\tau}^{a} & \text { averaged wall friction velocity: }\left(u_{\tau h}+u_{\tau c}\right) / 2 \\ u_{i}^{(\star)} & \text { estimated velocity } \\ U & \text { velocity scale of the flow } \\ W_{i j} & \text { differential tensorial function: } \lambda_{i j}-\tilde{\gamma}_{i j} \\ x_{i} & \text { coordinates }(i=1,2 \text { and } 3) \\ \Delta x_{i} & \text { grid size in the } x_{i} \text { direction }\end{array}$

\section{Greek Symbols}

$\begin{array}{ll}\alpha & \text { molecular thermal diffusivity: } \lambda /\left(\rho c_{P}\right) \\ \alpha_{i j} & \text { test-grid level base tensor: } 2 \tilde{\bar{\Delta}}^{2} \mid \tilde{\bar{S}}^{\prime} \tilde{\bar{S}}_{i j} \\ \alpha_{s g s} & \text { SGS eddy thermal diffusivity } \\ \beta_{i j} & \text { grid level base tensor: } 2 \bar{\Delta}^{2}|\bar{S}| \bar{S}_{i j} \\ \gamma_{i j} & \text { grid level base tensor: } 4 \bar{\Delta}^{2}\left(\bar{S}_{i k} \bar{\Omega}_{k j}-\bar{\Omega}_{i k} \bar{S}_{k j}\right) \\ \delta & \text { half channel height } \\ \delta_{i j} & \text { Kronecker delta } \\ \Delta & \text { mesh or filter size } \\ \epsilon & \text { dissipation rate of turbulent kinetic energy; } \\ & \text { or ratio between the cutoff sizes of the test-grid and grid filters: } \tilde{\bar{\Delta}} / \bar{\Delta} \\ \varepsilon_{i j k} & \text { Levi-Civita symbol } \\ \lambda & \text { thermal conductivity; or length scale of Taylor microscale } \\ \lambda_{i j} & \text { test-grid level base tensor: } 4 \tilde{\bar{\Delta}}^{2}\left(\tilde{\bar{S}}_{i k} \tilde{\bar{\Omega}}_{k j}-\tilde{\bar{\Omega}}_{i k} \tilde{\bar{S}}_{k j}\right)\end{array}$




$\begin{array}{ll}\zeta_{i j} & \text { test-grid level base tensor: } 4 \tilde{\bar{\Delta}}^{2}\left(\tilde{\bar{S}}_{i k} \tilde{\bar{S}}_{k j}-\frac{1}{3} \tilde{\bar{S}}_{m n} \tilde{\bar{S}}_{n m} \delta_{i j}\right) \\ \eta & \text { length scale for smallest eddies } \\ \eta_{i j} & \text { grid level base tensor: } 4 \bar{\Delta}^{2}\left(\bar{S}_{i k} \bar{S}_{k j}-\frac{1}{3} \bar{S}_{m n} \bar{S}_{n m} \delta_{i j}\right) \\ \nu & \text { kinematic viscosity } \\ \Omega & \text { angular velocity } \\ \bar{\Omega}_{i j} & \text { resolved rotation rate tensor: }\left(\partial \bar{u}_{i} / \partial x_{j}-\partial \bar{u}_{j} / \partial x_{i}\right) / 2 \\ \rho & \text { density } \\ \theta & \text { temperature } \\ \theta_{\tau} & \text { wall friction temperature: } q_{w} /\left(\rho c_{P} u_{\tau}\right) \\ \theta_{\tau}^{a} & \text { averaged wall friction temperature: }\left(\theta_{\tau h}+\theta_{\tau c}\right) / 2 \\ \tau & \text { time scale for energy containing eddies } \\ \tau_{w} & \text { wall shear stress } \\ \tau_{i j} & \text { grid level SGS stress tensor }\end{array}$

\section{Subscripts and Superscripts}

( ) $\quad$ streamwise component

( ) $\quad$ wall-normal component

( ) $3 \quad$ spanwise component

()$_{b} \quad$ value at the back face of a control volume

()$_{B} \quad$ value at the back neighbour node

( ) cold wall region

( ) e value at the east face of a control volume

()$_{E} \quad$ value at the east neighbour node

()$_{f} \quad$ value at the front face of a control volume

()$_{F} \quad$ value at the front neighbour node

()$_{h} \quad$ hot wall region

()$_{i},()_{j},()_{i j} \quad$ vectors or second-order tensors: $i, j=1,2,3$ 


$\begin{array}{ll}()_{i j}^{*} & \text { trace-free format of a second-order tensor: }()_{i j}^{*}=()_{i j}-()_{k k} \delta_{i j} / 3 \\ ()_{n} & \text { value at the north face of a control volume } \\ ()_{N} & \text { value at the north neighbour node } \\ ()_{N P} & \text { value at a neighbour node } \\ ()^{(n)} & \text { result at the } n \text {-th time step } \\ ()_{P} & \text { value at the central node } \\ ()_{s} & \text { value at the south face of a control volume } \\ ()_{S} & \text { value at the south neighbour node } \\ ()_{w} & \text { value at the west face of a control volume; } \\ ()_{W} & \text { or value at the wall } \\ \overline{(~)} & \text { value at the west neighbour node } \\ \widetilde{(~)} & \text { grid level filter; or a resolved quantity } \\ \widehat{(~)} & \text { test-grid level filter } \\ ()^{\prime \prime} & \text { Fourier transform } \\ ()^{+} & \text {residual component } \\ ()^{-} & \text {wall coordinates; or forward scatter } \\ {[]} & \text { backward scatter } \\ \langle\rangle & \text { timsemble-averaged quantity plane-averaged quantity }\end{array}$

\section{Abbreviations}

1-Dimensional

2-D

2-Dimensional

3-D

3-Dimensional

CAT

Clear Air Turbulence

CFD

Computational Fluid Dynamics

CFL

Courant-Friedrichs-Lewy 
DEDM Dynamic Eddy Diffusivity Model

DFLTDM Dynamic Full Linear Tensor Diffusivity Model

DHLTDM Dynamic Homogeneous Linear Tensor Diffusivity Model

DM Dynamic Smagorinsky Model

DNM Dynamic Nonlinear SGS Stress Model

DNS Direct Numerical Simulation

FV Finite Volume

HF Heat Flux

KE Kinetic Energy

LDV Laser-Doppler velocimetry

LES Large-Eddy Simulation

LHS Left-Hand-Side

MILES Monotonically Integrated Large-Eddy Simulation

NP Neighbour Points

N-S Navier-Stokes

PIV Particle Image Velocimetry

PTV Particle Tracking Velocimetry

RANS Reynolds-Averaged Navier-Stokes

RHS Right-Hand-Side

RMHF Resolved Molecular Heat Flux

RMS Root-Mean-Square

RTHF Resolved Turbulent Heat Flux

SGS Subgrid-Scale

SM Smagorinsky SGS Stress Model

TKE Turbulent Kinetic Energy

T-G Taylor-Görtler

ZPG Zero-Pressure Gradient 


\section{Chapter 1}

\section{Introduction}

\subsection{Turbulence: Basic Concepts and Research}

Turbulence is a physical phenomenon that occurs frequently in nature. It could appear in almost all flow media at an arbitrary speed ranging from almost stagnant fluids such as the clear-air turbulence (CAT) at high altitudes of the atmosphere to air flows in wind-tunnels at hypersonic and supersonic speeds. Turbulence phenomena can take place in inner flows in pipes, precipitators, gas scrubbers, heat exchangers, internal combustion engines and gas turbines, etc.; or in external flows over the surface of a moving object such as cars, airplanes, ships and submarines, soccer, tennis and golf balls. Although turbulence is rather a familiar terminology, a unique and unambiguous definition precisely that describes all characteristics of turbulence is still lacking. We can state that turbulence denotes a state of fluid in which all properties (velocity, pressure, density, etc.) fluctuate continuously in an irregular, chaotic, non-repeating and unpredictable manner [1].

Turbulence has been the subject of study for several centuries. However, it still remains an important unsolved problem of classical physics due to its complexity. In 1510, Leonardo da Vinci accomplished a drawing of the flow structures behind a blunt obstacle and wrote the following notes [2]: "Observation the motion of the water surface, which resembles that of hair, that has two motions: one due to the weight of the shaft, the other to the shape of the curls; thus, water has eddying motions, one 
part of which is due to the principal current, the other to the random and reverse motion." This observation is regarded as a precursor to Reynolds' decomposition of velocity, pressure and other variable into mean and fluctuation parts. In 1822, Claude L. M. H. Navier and George G. Stokes derived mathematical equations (socalled the Navier-Stokes (N-S) equations) that govern both laminar and turbulent flow motions. Although the equations for describing the turbulence were established, it was still impossible to obtain analytical or theoretical solutions related to turbulent flows because the N-S equations are highly nonlinear and non-integrable. In 1883, Osborne Reynolds [3] conducted a ground-breaking experiment that initiated the modern concept and scientific research of turbulence. In his experimental study, an important non-dimensional parameter was introduced to characterize the flow, the so-called Reynolds number, defined as $R e=U L / \nu$, where $U$ and $L$ are characteristic velocity and length scales of the flow, and $\nu$ is the kinematic viscosity of the fluid. In Reynolds's pipe-flow experiment, if $R e$ was less than about 2,300, the flow was laminar, however, if the value of $R e$ exceeded this threshold, transition to turbulence could occur.

During the last centuries, many leading researchers conducted research on turbulent flow phenomena using a variety of different approaches. The understanding of the scaling laws in various flow regimes is attributed to the research on statistical theories of turbulence. Experimental studies have played very important part in the understanding of the structure of turbulence, especially in identification of the coherent eddies responsible for the most prominent energy production. The advancement in the measurement technology has also facilitated experimental study of turbulence. Now, it is possible to obtain single-point measurements of velocity and velocity gradient components using laser-doppler velocimetry (LDV) and multiple wire anemometers, and velocity distributions in a plane using particle-image or particletracking velocimetry (PIV or PTV). Over the last 40 years, due to the advancement in the high-performance supercomputer technology and development in numerical algorithms such as direct and large-eddy simulation methods, data that could not be measured previously such as multi-point correlations, unobtrusive measurements 
of the velocity, velocity gradients, pressure, passive scalars, etc. can now obtained using these advanced numerical tools. Owing to the excellent temporal and spatial accuracy of the direct numerical simulation (DNS) method, the databases obtained from it have been extensively used for model validation and development. However, current computational capacity is still woefully insufficient in comparison with the needs for simulating complex engineering turbulent flows. [2]

\subsection{Characteristic Scales of Turbulence}

Owing to the fact that a precise definition of turbulence is still lacking, physical concepts supported by similarity arguments and dimensional analysis have been extensively used to characterize the turbulence and its properties in various flows.

In 1922, Richardson [4] introduced the seminal idea of "energy cascade" to describe the scales of turbulence. Kolmogorov [5] advanced this theory in 1941. According to the theory of Richardson and Kolmogorov, turbulence can be decomposed into eddies of different sizes. Turbulence and turbulence interactions are characterized by different temporal and spatial scales. Turbulence generation and its transport occur at large eddies, while the viscous dissipation is associated with the smallest scales (namely, the Kolmogorov scale). In a shear layer or in an enclosure, generation of turbulence occurs due to the mean flow deformation and its interaction with most energetic eddies (energy containing eddies). Large eddies grow until the layer or the entire flow domain is filled, thus the length of the largest scale is typically related to the characteristic flow dimension. Through the process of their generation, large eddies extract energy from the mean motion. The large eddies are unstable and break up, transferring their energy to somewhat smaller eddies. The small eddies undergo a similar break-up process, and transfer their energy to smaller and smaller eddies. Eventually, this energy will be dissipated by viscosity. There is an intermediate scale between large energetic scale and Kolmogorov scale, so-called Taylor microscale.

The dissipation rate of turbulent kinetic energy (TKE) is defined by $\epsilon$. The time 
TABLE 1.1: Turbulence scales

\begin{tabular}{l|cccc}
\hline Eddy size & Length scale & Time scale & Velocity scale & Reynolds No. \\
\hline Large energetic eddies & $l=\frac{k^{3 / 2}}{\epsilon}$ & $\tau=\frac{k}{\epsilon}$ & $k^{\frac{1}{2}}$ & $R e_{t}=\frac{k^{\frac{1}{2}} l}{\nu}$ \\
Small eddies & $\lambda=\sqrt{10 \nu \frac{k}{\epsilon}}$ & $\vartheta=\left(\frac{\nu}{\epsilon}\right)^{\frac{1}{2}}$ & $k^{\frac{1}{2}}$ & $R e_{\lambda}=\frac{k^{\frac{1}{2}} \lambda}{\nu}$ \\
Dissipative eddies & $\eta=\left(\frac{\nu^{3}}{\epsilon}\right)^{\frac{1}{4}}$ & $\vartheta=\left(\frac{\nu}{\epsilon}\right)^{\frac{1}{2}}$ & $(\nu \epsilon)^{\frac{1}{4}}$ & $R e_{k}=1$ \\
\hline
\end{tabular}

and length scales of the energy containing eddies can be defined as a function of TKE $k$ and its dissipation rate $\epsilon$, i.e. $f(k, \epsilon)$. Calculated according to the dimensional analysis, the time and length scales for energy containing eddies are $\tau=\frac{k}{\epsilon}$ and $l=\frac{k^{3 / 2}}{\epsilon}$, respectively. In the finest eddy structure (at the Kolmogorov scale), TKE converts to thermal energy through molecular dissipation. Thus, scales characteristic of the finest eddy structures are determined by two quantities, namely, $\nu$ and $\epsilon$. Dimensional analysis yields the following time and length scales: $\vartheta=\left(\frac{\nu}{\epsilon}\right)^{1 / 2}$ and $\eta=\left(\frac{\nu^{3}}{\epsilon}\right)^{\frac{1}{4}}$. The length scale of the Taylor microscale can be defined with respect to all three characteristic parameters, $k, \nu$ and $\epsilon$, which is $\lambda=\sqrt{10 \nu \frac{k}{\epsilon}}$. The characteristic time, length and velcoity scales and the corresponding Reynolds numbers for different eddies are summarized in the Table 1.1. The relevant relationships between various scales follow:

$$
\begin{aligned}
& \frac{l}{\lambda}=\frac{R e_{t}^{\frac{1}{2}}}{\sqrt{10}}=\frac{R e_{\lambda}}{10}, \\
& \frac{\lambda}{\vartheta}=\sqrt{10} R e_{t}^{\frac{1}{4}}=10^{\frac{1}{4}} R e_{\lambda}^{\frac{1}{2}}, \\
& \frac{l}{\eta}=R e_{t}^{\frac{3}{4}}=\frac{R e_{\lambda}^{\frac{3}{2}}}{10^{\frac{3}{4}}}, \\
& \frac{\tau}{\vartheta}=R e_{t}^{\frac{1}{2}}=\sqrt{10} R e_{\lambda} .
\end{aligned}
$$

\subsection{Numerical Simulation of Turbulence}

Although Navier-Stokes equations are believed to be capable of describing turbulent motions with very high degree of accuracy, there are infinite numbers of solution realizations that all satisfy the equations. There is no prospect of the existence of a simple 
analytic theroy. One of the hopes to solve the equations is to use the ever-increasing power of digital computers to achieve the objective of calculating the relevant properties of turbulent flows. [2] Direct numerical simulation (DNS), Reynolds averaged Navier-Stokes (RANS) approach and large-eddy simulation (LES) represent the three major methods in the field of numerical simulation of turbulent flows. In DNS, all turbulent motions are fully resolved including the finest Kolmogorov time and length scales. Although DNS provides the most accurate simulation results, the computational expenses associated with this method are very demanding. The computer requirements increase rapidly with the Reynolds number such that the applicability of the approach is still limited to flows of low or moderate Reynolds numbers, especially in the context of a complex domain. The RANS approach deals with the ensemble-averaged equations, only the largest energy-containing eddy motions are resolved at the large integral scales. Because the small and intermediate turbulent motions are not resolved in a RANS approach, their effects are modelled, leading to the special area of research so-called turbulence modelling. In a RANS approach, turbulence modelling plays a critically important role in numerical simulations, and the obtained numerical results are often sensitive to the specific turbulence modelling in use. Owing to the heavy empiricism involved in RANS models, they are short of the capacity of providing any accurate information about the turbulence spectral dynamics, and thus, a RANS approach does not have necessary temporal and spatial resolutions that are crucial for studying the physics of turbulence at small and intermediate scales.

The method of LES makes a compromise between DNS and RANS. In a RANS approach, turbulent motions at almost all scales (that are smaller than the integral scales) are modelled. In contrast, in LES, the large-scale eddy motions are resolved temporally and spatially. However, small-eddy motions at and below the subgrid scales are modelled. The large and small scale eddy motions are distinguished elegantly by a filtering process in a LES approach. Similar to the DNS, LES also provides a three-dimensional, time-dependent solution to the filtered governing equations. However, in LES, turbulent fields are resolved at and above a characteristic 
cut-off filter size, which is typically much larger than the Kolmogorov scales required in a DNS approach. In view of this, given the same computational resources, LES can deal with much higher Reynolds numbers and much more complex geometries than DNS.

\subsection{Subgrid-Scale Models in LES}

The LES represents a time-accurate numerical approach for improved predictions of complex turbulent flows. In LES, a flow is decomposed into large- and small-scale motions through a filter. The large-scale motions are typically energy containing and tend to be anisotropic. They dominate the transport processes of momentum, mass and thermal energy, and are directly computed in a LES approach. In contrast, the small-scale (or, subgrid-scale) motions tend to be more isotropic and less energetic, which usually need to be modelled.

As a consequence of the filtering process, two new terms appear in the system of governing equations for LES of thermal flows; namely, the subgrid-scale (SGS) stress tensor and SGS heat flux (HF) vector. In order to close the governing equations, both the SGS stress tensor and HF vector need to be modelled in a convential LES approach.

\subsubsection{Dynamic Modelling for SGS Stresses}

The first SGS stress model was proposed by Smagorinsky in 1963, now commonly referred to as the Smagorinsky model (SM) [6]. In this model, a model coefficient which is treated as a constant is introduced to correlate the SGS stress tensor $\tau_{i j}$ with the resolved strain rate tensor $\bar{S}_{i j}$ (defined as $\left.\bar{S}_{i j} \stackrel{\text { def }}{=}\left(\partial \bar{u}_{i} / \partial x_{j}+\partial \bar{u}_{j} / \partial x_{i}\right) / 2\right)$. Lilly [7] observed that the Smagorinsky model coefficient remains as a universal constant in homogeneous and isotropic turbulence, which is approximately 0.17. Unfortunately, it was soon discovered that this value needs to be modified to approximately 0.065 in the central core of a channel flow and reduced even further in regions close to a 
solid surface. Furthermore, the Smagorinsky coefficient is found to be a function of Reynolds number and other non-dimensional parameters instead of a constant $[8,9]$.

In 1991, Germano et al. [10] proposed the dynamic model (DM), which is regarded as a groundbreaking advancement in SGS stress modeling. Without utilizing any pre-determined empirical model coefficients and wall damping functions, the DM dynamically calibrates both the model coefficient and the near-wall SGS length-scale based on the updated resolved flow field. There are several different optimization principles to obtain the dynamic coefficient of the DM, among which the most popular one is the least squares method proposed by Lilly [7]. The DM has a few drawbacks related to the overly simplistic constitutive relation embedded in its modelling approach.

To overcome the difficulties related to the DM, Wang and Bergstrom [11] proposed an advanced dynamic nonlinear subgrid-scale stress model (DNM). The DNM is based on an explicit nonlinear quadratic tensorial polynomial constitutive relation of Speziale [12], which includes the conventional DM as its first-order approximation as well as two higher-order tensorial constituent components for nonlinear anisotropic representation of the SGS stress tensor. As a result, it can exhibit local stability without the need for plane-averaging, and reflect the physical mechanisms of both forward and backward scatter of SGS KE between the filtered and subgrid scale motions $[11,13,14]$. In comparison with the DM, the DNM overcomes several major drawbacks of the DM and admits more degrees of freedom for geometrical representation of the SGS stress tensor.

\subsubsection{Dynamic Modelling for SGS Heat Fluxes}

Shortly after the proposal of the dynamic SGS stress model by Germano et al. [10], the dynamic procedure was introduced to the development of SGS HF modelling for LES of turbulent scalar transport processes by Moin et al. [15]. They proposed a dynamic eddy thermal diffusivity model (DEDM) for representing the SGS HF vector. The DEDM based on a linear constitutive relationship between the SGS HF vectors 
and the resolved temperature gradient. Analogous to the Fourier's law for molecular heat conduction, this relationship assumes that the SGS HF vector is instantaneously proportional to and aligned with the negative of the resolved temperature gradient, i.e. $h_{j} \propto-\partial \bar{\theta} / x_{j}$. However, due to its overly simplified constitutive relation, this model cannot always correctly reflect the local geometrial properties of the SGS HF vector $[16,17]$. In spite of these deficiencies, the DEDM is still the most popular in literature and has been successfully applied to the prediction of many thermal convective flows [18-20].

Since the pioneering work of Moin et al. [15], the dynamic SGS HF modelling has progressed from models of the eddy thermal diffusivity type to those based on a tensor thermal diffusivity (or more briefly, tensor diffusivity, represented by $D_{i j}$ ) in order to further improve the physical and geometrical representation of the SGS HF vector. The explicit algebraic dynamic SGS HF modelling approach is represented by two types: featuring symmetrical and non-symmetrical tensor diffusivities $[9,21]$. For the symmetrical tensor diffusivity approach, the tensor diffusivity can be typically constructed as a tensor function of a readily available real symmetric tensor, e.g. the SGS stress tensor $\tau_{i j}$ or the resolved strain rate tensor $\bar{S}_{i j}$. In the conventinal model of Moin et al. [15], the eddy thermal diffusivity $\alpha_{s g s}$ is a scalar, which can be regarded as a symmetrical tensor of zeroth-order by using the Kronecker delta (viz., $\left.D_{i j}=\alpha_{s g s} \delta_{i j}\right)$. As an improvement, a dynamic homogenous linear tensor diffusivity model (DHLTDM) for representing the SGS heat flux was proposed by Peng and Davidson [22] in their study of a buoyancy-driven turbulent flow. In their approach, the tensor diffusivity is constructed as a homogenous linear function of the resolved strain rate tensor $\bar{S}_{i j}$. Wang et al. [9,21] systematically developed the algebraic dynamic SGS HF modelling approaches based on the strain rate tensor $\bar{S}_{i j}$ and SGS stress tensor $\tau_{i j}$ in their research, three variants of the constitutive formulas based on the homogeneous linear tensor function, full linear tensor function and quadratic tensor function of the constituent tensor $\left(\bar{S}_{i j}\right.$ and $\left.\tau_{i j}\right)$ have been thoroughly investigated. In their study, a dynamic full linear tensor diffusivity model (DFLTDM) was proposed by Wang et al. [9]. It is reported that this model has a relative simple con- 
stitutive relation while exhibiting excellent predictive performance. In view of this, in this research, the DFLTDM is chosed to model the SGS HF vector.

\subsection{Motivation}

Numerical simulation of heated rotating turbulent flows is an interesting topic in computational fluid dynamics (CFD) with many important engineering applications such as gas turbines, rotating heat exchangers, and centrifugal pumps and fans. The prediction and analysis of the effects of rotation on a thermal flow field is crucial in designing high-performance rotating devices involving heat transfer. Due to the system rotation, two additional body forces, i.e. the centrifugal and Corilois forces are generated. The mean flow and turbulence level in a rotating channel are influenced significantly by the Coriolis and centrifugal forces associated with the system rotation. In response to the Coriolis and centrifugal forces, large secondary flows are induced, which then dramatically alter the turbulent flow structures and the process of heat and mass transfer. As a consequence, the physical mechanisms underlying the transport of momentum and mass in rotating flows are subjected to further dynamical complexities, which impose additional challenges on the predictive accuracy of turbulence models.

In engineering applications, a rotary machine typically rotates in the designed direction with specific flow paths. An arbitrary directional rotation vector can be decomposed into componential rotation vectors in three orthogonal directions. The simplest flow in this category is a fully-developed turbulent flow in a rotating plane channel, where the rotating axis is parallel to one of the three axial directions: the streamwise, wall-normal, and spanwise directions.

Among the studies of turbulent channel flows subjected to these three types of system rotations, the spanwise rotating turbulent channel flows have been studied extensively through experiments $[23,24]$ and numerical simulations $[14,25-37]$. It is reported that as the rotation number increases, turbulence is gradually enhanced on 
the pressure side and reduced on the suction side, further resulting in asymmetric distributions in the mean flow and Reynolds stresses [14,23-37]. At the same time, large-scale roll cells come forth as a result of the Taylor-Görtler (T-G) instability $[14,23,25,35]$. These large-scale roll cells dominate the secondary flow pattern in a channel, and shift towards the pressure side. The number of the pairs of such cells tends to increase as the rotation number increases [35]. At high rotation numbers, turbulence on the suction side reduces significantly, and the roll cells become much smaller and eventually disappear due to thickening of the relaminarized region on the suction side $[36,37]$. For a heated turbulent channel flow subjected to spanwise rotations, the Nusselt number also increases on the pressure side and decreases on the suction side [38]. The effects of rotation on the thermal diffusive sublayer near the pressure and suction sides are consistent with those characteristic of the momentum viscous sublayer. This phenomenon is typically referred to as the 'Ekman layer effect', and is caused by the balance between the Coriolis force, mean pressure gradient and turbulent drag in a layer of flow subjected to a system rotation. Owing to the existence of the Coriolis force, large T-G vortices are induced in the core region of the channel, which shift both the mean temperature and mean velocity profiles to the pressure side. Consequently, both the momentum viscous sublayer and the thermal diffusive sublayer become thinner on the pressure side and become thicker on the suction side [14]. Furthermore, in the recent work of Xun et al. [39], it was found that the appearance of the T-G vortices causes quasi-periodicity in the spanwise distribution of the drag coefficient and Nusselt number.

A turbulent channel flow subjected to wall-normal system rotations can be regarded as a prototype of a three dimensional boundary layer. However, this type of rotating channel flow has been rarely investigated, and even if studied, the focus is on very small rotation rates. Elsamni and Kasagi [36,38] and Wu and Kasagi [40] studied the effects of arbitrary direction system rotations on a turbulent channel flow. In their study, it is observed that in the context of a wall-normal rotating flow, the velocity field is very sensitive to the rotation rate imposed upon the flow. Li et al. [41] also studied the effects of wall-normal rotation on a turbulent channel flow driven by a 
constant pressure gradient. They also reported that the channel flow is very sensitive to the wall-normal rotation. A slight rotation in the wall-normal direction can induce a strong secondary motion in the spanwise direction and reduce the streamwise mean velocity substantially. As a result, all six Reynolds stress components become nonzero, which further redirect the mean shear distribution and reform the turbulent structures. The interaction between the vorticity induced coherent structures and the background vorticity in the context of wall-normal rotating flow can significantly alter the near-wall turbulence behavior. Furthermore, it is confirmed in the study of Li et al. [41] that flow structures are more sensitive to the Coriolis force effect induced by the wall-normal rotation. Mehdizadeh [42] investigated wall-normal rotating channel flows over a wide range of rotation rates using the DNS approach. He reported that as the rotation rate increases, relaminarization effects appear, and finally at very high rotation rates, the flow reaches a fully laminar steady state.

Turbulent heat, mass and fluid flows subjected to streamwise system rotations have been intensively investigated only recently in comparison with the extensively studied spanwise turbulent channel flows. Turbulent channel flow subjected to heating and streamwise rotation is one of the important benchmark test cases for examining the predictive accuracy of the turbulent models in the context of non-inertial coordinate system. Analysis of this type of flow based on the Lie-group theory and DNS $[40,43]$ has shown that a secondary flow perpendicular to the mean flow direction is usually generated, whose strength strongly depends upon the streamwise rotating speed. The existence of this secondary flow in the spanwise direction has been recently confirmed by the PIV measurements of Recktenwald et al. [44]. EI-Samni et al. [45] and Weller and Oberlack [46] analyzed the structures of turbulent flows subjected to streamwise rotation using the method of DNS. Recktenwald et al. [47] further investigated the influence of streamwise rotation on a turbulent channel flow based on an analysis of both DNS and PIV data.

The method of LES is effective in resolving the evolution of a thermal-fluid field at scales that are equal to or larger than a certain filter size. In a conventional implicitfiltering LES approach, use of SGS stress and HF models is necessary for the closure of 
governing equations. This is in sharp contrast to the monotonically integrated largeeddy simulation (MILES) approach used by Alkishriwi et al. [48], who conducted numerical investigation of streamwise rotating channel flows based on MILES. In MILES, the effects of SGS physics on the resolved scales are not represented by any explicit SGS models, instead, they are elegantly incorporated in the functional reconstruction of the convective fluxes using locally monotonic methods [49]. Although the conventional implicit-filtering approach has been the most popular in community, there are serious debates on whether the MILES or the conventional implicit-filtering LES is a more effective approach for numerical simulations. In this research, we use exclusively the conventional implicit-filtering LES approach to conduct numerical simulations. As a result, SGS stress and heat flux models are needed for closure of the filtered momentum and thermal energy equations, respectively.

In the literature related to the conventional implicit-filtering LES, dynamic SGS stress models have been utilized for numerical simulation of rotating channel flows. Oberlack et al. [43] investigated the effect of the Coriolis force on the mean and turbulent flow structures using the dynamic SGS stress model (DM) of Germano et al. [10] and Lilly [7], and successfully reproduced most of the DNS results at the filtered scale. Piomelli and Liu [28] proposed a localized dynamic Smagorinsky type SGS stress model which computed the model coefficient using a backward extrapolation scheme. Lamballais et al. [37] developed a spectral-dynamic SGS stress model based on the eddy-damped quasi-normal Markovian statistical theory. Pallares and Davidson [18] and Pallares et al. [50] utilized the one-equation dynamic SGS model of Kim and Menon [51] in their numerical study of turbulent flow and heat transfer in rotating square ducts. Different from the previous approaches, in this research, two innovative dynamic explicit algebraic SGS stress and HF models will be used for improved LES of turbulent thermal flows subjected to streamwise rotations. The novel dynamic SGS modelling approaches adopted by this research are to be briefly introduced in next subsection. 


\subsection{Objectives of the Thesis}

This research includes three major objectives:

i) The first objective of this thesis is to study the flow structure of a heated plane channel subjected to streamwise system rotations. In order to examine the effect of centrifugal and Coriolis forces associate with the system rotation on the coupled thermal flow fields, the first- and second-order statistics of the velocity and temperature fields characteristic of heated rotating channel flows at different rotation numbers are thoroughly analyzed. This includes the analysis of the mean resolved velocity, mean resolved temperature and the associated second-order statistics such as the fluctuations of the resolved velocity and temperature, TKE, turbulent stresses and heat fluxes.

ii) To date, the advanced DNM of Wang and Bergstrom [11] for modelling the SGS stress tensor and the DFLTDM of Wang et al. [9] for modelling the SGS HF vector, have only been tested using a few canonical test cases such as Couette and Poiseuille flows, mixed natural and forced convection flows in horizontal and vertical channels $[9,21]$, and physiological pulsatile transition-to-turbulent flows in vessels with idealized arterial stenosis [52]. Xun et al. [14] thoroughly examined the performance of these advanced modelling approaches in the context of a heated rotating channel flow subjected to spanwise rotations. Because both the centrifugal force and the two Coriolis force components are sensitive to the direction of system rotations [53,54], the flow physics in the streamwise and spanwise rotating channel flows are significantly different (the secondary flow is dominated by T-G vortices in a spanwise rotating flow, however, by four opposite stratification layers in a streamwise rotating flow). In view of this, in this research, this reseasrch aims at examining the predictive performance of these two advanced SGS stress and HF models (viz., the DNM and DFLTDM, respectively) in the context of a heated channel flow subjected to streamwise rotations.

iii) In this thesis, a variety of rotation numbers ranging from $R o_{\tau}=0$ to 15 have been tested in conjunction with two fixed low Reynolds numbers $R e_{\tau}=150$ and 300 . 
The final objective of this work is to investigate the influence of Reynolds number on streamwise system rotating channel flows, and further examine the performance of these two advanced models (DNM and DFLTDM) in response to changes in the Reynolds number.

\subsection{Outline of the Thesis}

The thesis is organized as follows. The methodology of LES is introduced in chapter 2, which covers the filtering operation for LES, the algorithm for solving the filtered governing equations, and SGS stress and HF models to be used for closure of the filtered momentum and scalar transport equations.

In chapter 3 , the effects of the streamwise rotation on the thermal-fluid fields at different Reynolds numbers are investigated based on five different rotation numbers, varying from a non-rotating case $\left(R o_{\tau}=0\right)$ to a relatively strong rotation case $\left(R o_{\tau}=15.0\right)$. In order to validate the algorithm and several innovative SGS modelling approaches adopted in this research, numerical results obtained from the LES are compared against the reported DNS data. Furthermore, in order to examine the performance of the two dynamic SGS stress models (i.e., the DM and DNM), a detailed comparative study is performed. The fundamental flow physics of the turbulent flow under system rotations at two different Reynolds numbers are systematically analyzed, including the mean and RMS values of the resolved velocity and temperature fields.

In chapter 4, a further study of the LES approach and the associated modelling effects are conducted by thoroughly analyzing some advanced physical features in terms of the transport of turbulent stresses, TKE and heat fluxes, and forward and backward scatter of local KE fluxes between the resolved and subgrid scales. The rotation effects on these advanced physical features are also examined by comparing the numerical results obtained at different rotation numbers.

In chapter 5, a summary of the major conclusions of this thesis and a discussion 
of the directions for possible future explorations are presented. 


\section{Chapter 2}

\section{LES Algorithm and Test Case}

\section{$2.1 \quad$ Filtering Operation}

\subsubsection{Basic Concepts}

As introduced in previous chapter, LES is based on the notion that turbulent flows contain a wide range of length and time scales. Usually, a filtering operation is used to separate large and small scales in LES. A filtered variable, which indicates a resolved (or, large-scale) variable, is denoted using an overbar, defined as

$$
\bar{f}(x)=\int_{D} f\left(x^{\prime}\right) G\left(x, x^{\prime}\right) d x^{\prime}
$$

where $D$ represents the domain and $G(x, y)$ is the filter function. The filter function determines the size of the small scales. There are three most commonly used filter functions, including: the sharp spectral (Fourier cutoff) filter,

$$
\hat{G}(k)=\left\{\begin{array}{ll}
1 & \text { if } k \leq \pi \bar{\Delta} \\
0 & \text { otherwise }
\end{array},\right.
$$

the Gaussian filter,

$$
G(x)=\sqrt{\frac{6}{\pi \bar{\Delta}^{2}}} \exp \left(-\frac{6 x^{2}}{\bar{\Delta}^{2}}\right)
$$

and the box (top hat) filter, 


$$
G(x)=\left\{\begin{array}{cl}
1 / \bar{\Delta} & \text { if }|x| \leq \bar{\Delta} / 2 \\
0 & \text { otherwise }
\end{array}\right.
$$

where a caret $(\hat{\cdot})$ denotes the Fourier transform of a variable, $k$ represents the wavenumber in the Fourier space, and $\bar{\Delta}$ denotes the filter width.

Pope [55] pointed out that the sharp spectral filter annihilates all Fourier modes of wavenumber $|k|$ greater than the cutoff wavenumber $k_{c}=\pi / \bar{\Delta}$ (which is evident in Eq. (2.2). However, it has no effect on lower wavenumber modes. Furthermore, although it is "sharp" in the wavenumber space, the sharp spectral filter is non-local in the physical space. The property of the box filter is just opposite to that of the sharp spectral filter. Among the three filters discussed above, the Gaussian filter is reasonably compact both in the physical and in the wavenumber spaces.

\subsubsection{Discrete Filtering Process}

In a dynamic SGS modelling approach, the test-grid level filtering process requires an explicit discrete filter. Throughout this thesis, the discrete Gaussian filters introduced by Sagaut and Grohens [56] are used to perform the filtering process at the test-grid level.

In Sagaut and Grohens [56], two methods were proposed to construct a threedimensional (3-D) filtering convolution based on a one-dimensional (1-D) discrete filter. One is the linear combination method, and the other is the so-called product method. The discrete convolution constructed using the linear combination method has the following form:

$$
\tilde{f}(I, J, K)=\frac{1}{3} \sum_{l=-N}^{N} a_{l}[f(I+l, J, K)+f(I, J+l, K)+f(I, J, K+l)]
$$

while that constructed using the product method has the following form:

$$
\tilde{f}(I, J, K)=\sum_{l=-N}^{N} \sum_{m=-N}^{N} \sum_{n=-N}^{N} a_{l} a_{m} a_{n}[f(I+l, J+m, K+n)]
$$


where $N=1$ for a three-point stencil (2nd-order accuracy) and $N=2$ for a five-point stencil (4th-order accuracy), and $a_{l}, a_{m}$ and $a_{n}$ represent the filter coefficients for the 1-D discrete filter.

It is apparently that the cost of the product method is about two orders higher than that of the linear combination method. In LES, the linear combination method is more often used for building a discrete filter. In this study, without an otherwise specification, a 2nd-order discrete Gaussian filter is applied to the boundary field, while a 4th-order discrete Gaussian filter is used to deal with the internal field. The 1-D discrete forms for the 2nd- and 4th-order Gaussian filters given by Sagaut and Grohens [56] are, respectively,

$$
\tilde{f}(I)=\frac{1}{24} \epsilon^{2}[f(I+1)+f(I-1)]+\frac{1}{12}\left(12-\epsilon^{2}\right) f(I)
$$

and

$$
\begin{aligned}
\tilde{f}(I) & =\frac{\epsilon^{4}-4 \epsilon^{2}}{1152}[f(I+2)+f(I-2)]+\frac{16 \epsilon^{2}-\epsilon^{4}}{288}[f(I+1)+f(I-1)] \\
& +\frac{\epsilon^{4}-20 \epsilon^{2}+192}{192} f(I)
\end{aligned}
$$

where $\epsilon=\tilde{\bar{\Delta}} / \bar{\Delta}$ is the ratio between the cutoff sizes of the test-grid and grid filters. Following the conventional approach [10], it is set to $\epsilon=2$. With the choice of $\epsilon=2$, the discrete filtering scheme of 2nd-order accuracy (Eq. (2.7)) becomes identical to that of 4th-order accuracy (Eq. (2.8)).

\section{$2.2 \quad$ Filtered Governing Equations}

In LES, the filtered continuity, momentum and thermal energy equations take the following form for an incompressible flow in a domain subjected to a system rotation [57]:

$$
\frac{\partial \bar{u}_{i}}{\partial x_{i}}=0
$$




$$
\frac{\partial \bar{u}_{i}}{\partial t}+\frac{\partial\left(\bar{u}_{i} \bar{u}_{j}\right)}{\partial x_{j}}=-\frac{1}{\rho} \frac{\partial \bar{p}}{\partial x_{i}}+\nu \frac{\partial^{2} \bar{u}_{j}}{\partial x_{j} \partial x_{j}}-\frac{\partial \tau_{i j}}{\partial x_{j}}+2 \varepsilon_{i j k} \Omega_{k} \bar{u}_{j}-\left(\Omega_{i} \Omega_{k} x_{k}-\Omega_{k} \Omega_{k} x_{i}\right)
$$

$$
\frac{\partial \bar{\theta}}{\partial t}+\frac{\partial}{\partial x_{j}}\left(\bar{u}_{j} \bar{\theta}\right)=\alpha \frac{\partial^{2} \bar{\theta}}{\partial x_{j} \partial x_{j}}-\frac{\partial h_{j}}{\partial x_{j}}
$$

where $\bar{u}_{i}$ is the filtered velocity, $\bar{p}$ is the filtered pressure, $\Omega_{i}$ represents a constant positive angular velocity component of the system rotation in the $x_{i}$ coordinate direction, $x_{i}$ is the coordinate in space. In this thesis, $x_{1}, x_{2}$, and $x_{3}$ are used to denote the streamwise, wall-normal and spanwise coordinates, respectively (see Fig. 2.2). $\bar{\theta}$ represents the filtered temperature, and $\alpha$ is the molecular thermal diffusivity. $\varepsilon_{i j k}$ is the Levi-Civita symbol, which is defined by:

$$
\epsilon_{i j k}=\left\{\begin{array}{rcc}
1, & \text { for } \quad i j k=123,231,312 \\
-1, & \text { for } \quad i j k=321,132,213 \\
0, & \text { otherwise }
\end{array} .\right.
$$

The last two terms on the right hand side (RHS) of Eq. (2.10) represent the Coriolis and centrifugal force terms, respectively. The centrifugal forces can be expressed as a potential field, and in the context of incompressible flow, can be absorbed into the pressure term by introducing the concept of effective pressure

$$
\bar{p}_{e f f}=\bar{p}+\frac{1}{2} \rho\left(\Omega_{k} \Omega_{i} x_{k} x_{i}-\Omega_{k} \Omega_{k} x_{i} x_{i}\right)
$$

Under a streamwise system rotation, the filtered momentum equation can then be expressed as

$$
\frac{\partial \bar{u}_{i}}{\partial t}+\frac{\partial\left(\bar{u}_{i} \bar{u}_{j}\right)}{\partial x_{j}}=-\frac{1}{\rho} \frac{\partial \bar{p}}{\partial x_{i}}+\nu \frac{\partial^{2} \bar{u}_{i}}{\partial x_{j} \partial x_{j}}-\frac{\partial \tau_{i j}}{\partial x_{j}}+2 \varepsilon_{1 i j} \Omega \bar{u}_{j}
$$

In order to keep notations succinct in the discussion, $\bar{p}_{\text {eff }}$ is simply represented using $\bar{p}$ in the remainder of this thesis.

There are two terms appeared in the above system of governing equation as a 
consequece of the filtering process, namely, the SGS stress tensor and SGS HF vector, which are defined as

$$
\tau_{i j} \stackrel{\text { def }}{=} \overline{u_{i} u_{j}}-\bar{u}_{i} \bar{u}_{j}
$$

and

$$
h_{j} \stackrel{\text { def }}{=} \overline{u_{j} \theta}-\bar{u}_{j} \bar{\theta}
$$

respectively. In order to close the above system of governing equations, both the SGS stress tensor and HF vector need to be modelled.

\subsection{SGS Stress Models}

In this thesis, two SGS stress models are used for the closure of the filtered momentum equations: namely, the conventional dynamic Smagorinsky model (DM) of Lilly [7] and the novel dynamic nonlinear SGS stress model (DNM) of Wang and Bergstrom [11]. The following section briefly describes the formulations of these two dynamic linear and nonlinear models.

\subsubsection{SGS Stress Model 1 (DM)}

The conventional DM proposed by Lilly [7] has been widely used in the LES community due to its simplicity and robustness. The constitutive relation for the DM is based on a linear tensorial function of the resolved strain rate tensor $\bar{S}_{i j}$, i.e.

$$
\tau_{i j}^{*}=\tau_{i j}-\frac{\tau_{k k}}{3} \delta_{i j}=-2 C_{S} \bar{\Delta}^{2}|\bar{S}| \bar{S}_{i j}
$$

where $\bar{\Delta}$ is the grid level filter size, $\bar{S}_{i j} \stackrel{\text { def }}{=}\left(\partial \bar{u}_{i} / \partial x_{j}+\partial \bar{u}_{j} / \partial x_{i}\right) / 2$ is the resolved strain rate tensor, $|\bar{S}|=\left(2 \bar{S}_{i j} \bar{S}_{j i}\right)^{1 / 2}$ is the norm of the resolved strain rate tensor, $\delta_{i j}$ is the Kronecker delta, and an asterisk represents a trace-free tensor, i.e., $(\cdot)_{i j}^{*}=$ $(\cdot)_{i j}-(\cdot)_{k k} \delta_{i j} / 3$. Contrary to inputting a priori model coefficient $C_{S}$ in the SM, the optimal model coefficient $C_{S}$ can be obtained using the updated flow field dynamically. In the dynamic SGS modelling process proposed by Germano et al. [10], a second 
coarser test-grid level filtering process was introduced. After the second test-grid level filter (denoted by a tilde $\widetilde{(\cdot)}$ ) was applied to the filtered governing equations, a new SGS stress tensor at the test-grid level appears, which is defined as

$$
T_{i j} \stackrel{\text { def }}{=} \widetilde{u_{i} u_{j}}-\tilde{\bar{u}}_{i} \tilde{\bar{u}}_{j}
$$

The constitutive relation for the SGS stress tensor at the test-grid level is analogous to the constitutive relation for the SGS stress tensor at the grid level (i.e., Eq. (2.17)), which can be modelled as

$$
T_{i j}^{*}=-2 C_{S} \tilde{\bar{\Delta}}^{2}|\tilde{\bar{S}}| \tilde{\bar{S}}_{i j}
$$

Here, $\tilde{\bar{\Delta}}$ denotes the filter size of the test-grid filter. Following the suggestion of Germano et al. [10], the ratio of the width of the test-grid filter to that of the grid filter is typically set to 2 . The Germano identity [10] is defined based on the subtraction of the test-grid filtered $\tau_{i j}$ from $T_{i j}$, i.e.

$$
\mathcal{L}_{i j}=T_{i j}-\tilde{\tau}_{i j}=\widetilde{\bar{u}_{i} \bar{u}_{j}}-\tilde{\bar{u}}_{i} \tilde{\bar{u}}_{j}
$$

Substituting Eqs. (2.17) and (2.19) into Eq. (2.20), the following equation is obtained

$$
\mathcal{L}_{i j}-\frac{1}{3} \mathcal{L}_{k k}=-\alpha_{i j} C_{S}+\widetilde{\beta_{i j} C_{S}}
$$

where

$$
\alpha_{i j} \stackrel{\text { def }}{=} 2 \tilde{\bar{\Delta}}^{2}|\tilde{\bar{S}}| \tilde{\bar{S}}_{i j}
$$

and

$$
\beta_{i j} \stackrel{\text { def }}{=} 2 \bar{\Delta}^{2}|\bar{S}| \bar{S}_{i j}
$$

are the test-grid and grid level base stress tensors, respectively. Note that $\mathcal{L}_{i j}$ can be numerically calculated and thus the coefficient $C_{S}$ is the only unknown variable in Eq. (2.21). However, there are five independent equations available to determine a single coefficient $C_{S}$, implying that Eq. (2.21) is over-determined. Therefore, we can only approximately satisfy the Germano identity Eq. (2.20) through optimization. The error is

$$
e_{i j}=\mathcal{L}_{i j}^{*}+\alpha_{i j} C_{S}-\widetilde{\beta_{i j} C_{S}}
$$


where $\mathcal{L}_{i j}^{*}$ is the trace free form of $\mathcal{L}_{i j}$, i.e. $\mathcal{L}_{i j}^{*}=\mathcal{L}_{i j}-\frac{1}{3} \mathcal{L}_{k k}$. By assuming that the coefficient $C_{S}$ is spatially invariant within the test-grid filter, $C_{S}$ can be extracted from the test-grid filtering operation. Eq. (2.24) then becomes

$$
e_{i j}=\mathcal{L}_{i j}^{*}+C_{S} M_{i j}
$$

where

$$
M_{i j} \stackrel{\text { def }}{=} \alpha_{i j}-\tilde{\beta}_{i j}
$$

is a differential tensor. The least squares method can then be used to minimize the error of the Germano identity to obtain an optimal value for $C_{S}$ :

$$
\frac{\partial E}{\partial C_{S}}=\frac{\partial\left(e_{i j} e_{i j}\right)}{\partial C_{S}}=0
$$

For an incompressible flow, $\bar{S}_{i i}=0$, and therefore, the trace of $\mathcal{L}_{i j}$ vanishes (i.e., $\mathcal{L}_{i j}=\mathcal{L}_{i j}^{*}$ ). Solving Eq. (2.27), the expression for the model coefficient $C_{S}$ is obtained:

$$
C_{S}=-\frac{M_{i j} \mathcal{L}_{i j}}{M_{i j} M_{i j}}
$$

The derivation of other dynamic SGS stress models (e.g. the DNM) and SGS HF models (e.g. the DEDM and DFLTDM) with more than one coefficient could also be performed by following the same principle of the dynamic procedure demonstrated here.

Over the past 20 years, the DM has been successfully applied to prediction of many turbulent flows $[10,15,28,58,59]$. However, the DM uses the Smagorinsky constitutive relation in its constitutive relation, which results in a few drawbacks. For example, it can lead to an unrealistic SGS dissipation effect if the model coefficient is restricted to be positive; on the other hand, a potential numerical instability arises due to the excessive backscatter of the SGS if the model coefficient is allowed to be negative. Furthermore, the DM can be potentially ill-conditioned because the model coefficient is not bounded and admits a possible singularity when the denominator of the formulation $\left(M_{i j} M_{i j}\right)$ becomes very small [11]. Finally, this model requires the principal axes of the SGS stress tensor to be aligned with those of the resolved 
strain rate tensor, which leads to insufficient representation of the SGS stress components [13]. In order to avoid numerical instability due to either excessive backscatter or potential singular situation of the modelling formulation, a plane averaging technique is often adopted for the DM (when a homogeneous plane exists) [10,15,28,59]. For this approach, the above equation for determining the dynamic coefficient $C_{S}$ is revised to

$$
C_{S}=-\frac{\left\langle M_{i j} \mathcal{L}_{i j}\right\rangle}{\left\langle M_{i j} M_{i j}\right\rangle}
$$

where $\langle\cdot\rangle$ represents a quantity averaged both in time and over the homogeneous $\left(x_{1}, x_{3}\right)$-plane. However, there are two disadvantages related to the plane averaging scheme: firstly, it is difficult to apply a plane averaging scheme in flows that do not possess directions of statistical homogeneity; secondly, the plane averaging scheme will smear the local characteristics of the model.

\subsubsection{SGS Stress Model 2 (DNM)}

The dynamic nonlinear model (DNM) proposed by Wang and Bergstrom [11] overcomes some of the major drawbacks of the DM. The constitutive relationship for the DNM is based on an explicit nonlinear quadratic tensorial polynomial constitutive relation originally proposed by Speziale [12] for modeling of the Reynolds stress tensor in a RANS approach. Within the context of LES approach, the SGS stress tensor can be modelled by analogy using the following function:

$$
\tau_{i j}^{*}=-C_{S} \beta_{i j}-C_{W} \gamma_{i j}-C_{N} \eta_{i j}
$$

where the base tensor functions are defined as $\gamma_{i j} \stackrel{\text { def }}{=} 4 \bar{\Delta}^{2}\left(\bar{S}_{i k} \bar{\Omega}_{k j}+\bar{S}_{j k} \bar{\Omega}_{k i}\right)$ and $\eta_{i j} \stackrel{\text { def }}{=}$ $4 \bar{\Delta}^{2}\left(\bar{S}_{i k} \bar{S}_{k j}-\bar{S}_{m n} \bar{S}_{n m} \delta_{i j} / 3\right)$. Here, $\bar{\Omega}_{i j} \stackrel{\text { def }}{=}\left(\partial \bar{u}_{i} / \partial x_{j}-\partial \bar{u}_{j} / \partial x_{i}\right) / 2$ is the resolved rotation rate tensor. As introduced in [11], the least squares method can be used to obtain the values of the three dynamic model coefficients $C_{S}, C_{W}$ and $C_{N}$, viz. 


$$
\left[\begin{array}{ccc}
M_{i j} M_{i j} & M_{i j} W_{i j} & M_{i j} N_{i j} \\
W_{i j} M_{i j} & W_{i j} W_{i j} & W_{i j} N_{i j} \\
N_{i j} M_{i j} & N_{i j} W_{i j} & N_{i j} N_{i j}
\end{array}\right] \cdot\left[\begin{array}{c}
C_{S} \\
C_{W} \\
C_{N}
\end{array}\right]=-\left[\begin{array}{c}
\mathcal{L}_{i j}^{*} M_{i j} \\
\mathcal{L}_{i j}^{*} W_{i j} \\
\mathcal{L}_{i j}^{*} N_{i j}
\end{array}\right]
$$

where $W_{i j} \stackrel{\text { def }}{=} \lambda_{i j}-\tilde{\gamma}_{i j}$ and $N_{i j} \stackrel{\text { def }}{=} \zeta_{i j}-\tilde{\eta}_{i j}$ are differential tensors, with $\lambda_{i j} \stackrel{\text { def }}{=}$ $4 \tilde{\bar{\Delta}}^{2}\left(\tilde{\bar{S}}_{i k} \tilde{\bar{\Omega}}_{k j}+\tilde{\bar{S}}_{j k} \tilde{\bar{\Omega}}_{k i}\right)$ and $\zeta_{i j} \stackrel{\text { def }}{=} 4 \tilde{\bar{\Delta}}^{2}\left(\tilde{\bar{S}}_{i k} \tilde{\bar{S}}_{k j}-\tilde{\bar{S}}_{m n} \tilde{\bar{S}}_{n m} \delta_{i j} / 3\right)$.

The constitutive relation of Eq. (2.30) includes three tensorial constituent base components, i.e. $\beta_{i j}, \gamma_{i j}$ and $\eta_{i j}$. The first term $\beta_{i j}$ is the well-known Smagorinsky component which primarily relates to the SGS dissipation and forward scatter of TKE from the resolved to SGS motions; the second term $\gamma_{i j}$ does not make any contribution to the TKE transfer between the resolved and subgrid scales, but it significantly improves the correlation between the exact $\tau_{i j}$ extracted from a DNS database and that predicted by the nonlinear model; and the third term $\eta_{i j}$ contributes significantly to the backscatter of TKE from the subgrid to the resolved scales [11]. The three features mentioned above, namely, an adequate SGS dissipation level, a high correlation coefficient between the model and exact SGS stresses in a priori LES test, and a realistic representation of the TKE backscatter, are among the most important criteria for developing a high-fidelity SGS stress model. The constitutive relation of Eq. (2.30) offers an effective representation for modelling these three important physical features separately using three independent tensorial terms. Further investigation and application of the DNM can be found in Wang et al. [9,21] on turbulent channel flows with and without heat transfer, in Xun et al. [14] for Poiseuille type plane channel flows with and without spanwise system rotations, in Wang et al. [13] on the study of the geometrical properties of the SGS stress tensor, in Wang et al. [60] on the study of the topological features of wall-bounded turbulent flows and recently, in Molla et al. [52] on a physiological pulsatile transition-to-turbulent flow in an idealized blood vessel with stenosis. 


\subsection{SGS Heat Flux Models}

In this thesis, two dynamic SGS HF models are used: namely, the dynamic eddy diffusivity model (DEDM) of Moin et al. [15] and the dynamic full linear tensor diffusivity model (DFLTDM) of Wang et al. [61].

\subsubsection{SGS Heat Flux Model 1 (DEDM)}

The SGS heat flux in the DEDM proposed by Moin et al. [15] is expressed as

$$
h_{j}=-C_{\theta P} \bar{\Delta}^{2}|\bar{S}| \frac{\partial \bar{\theta}}{\partial x_{j}}
$$

where $\alpha_{s g s}=C_{\theta P} \bar{\Delta}^{2}|\bar{S}|$ is the scalar eddy diffusivity embodied in Eq. (2.32). By using the Kronecker delta, it can be further written in a general tensor diffusivity form as

$$
D_{j k}^{E}=\alpha_{s g s} \delta_{j k}=C_{\theta P} \bar{\Delta}^{2}|\bar{S}| \delta_{j k}
$$

With this notion, Eq. (2.32) can be simplified to $h_{j}=-D_{j k}^{E} \frac{\partial \bar{\theta}}{\partial x_{k}}$. Define $b_{j}^{P} \stackrel{\text { def }}{=}$ $\bar{\Delta}^{2}|\bar{S}| \frac{\partial \bar{\theta}}{\partial x_{j}}$ and $a_{j}^{P} \stackrel{\text { def }}{=} \tilde{\bar{\Delta}}^{2}|\tilde{\bar{S}}| \frac{\partial \tilde{\tilde{\theta}}}{\partial x_{j}}$ as the grid level and test-grid level base vector functions. The SGS HF vector at the grid level represented by Eq. (2.32) can then be expressed as $h_{j}=-C_{\theta P} b_{j}^{P}$. Similarly, the SGS HF vector at the test-grid level $\left(H_{j} \stackrel{\text { def }}{=} \widetilde{\widetilde{u_{j} \theta}}-\tilde{\bar{u}}_{j} \tilde{\bar{\theta}}\right)$ can be modelled as $H_{j}=-C_{\theta P} a_{j}^{P}$. The grid and test-grid level SGS HF vectors satisfy the vector identity:

$$
\mathcal{L}_{j}=H_{j}-\tilde{h}_{j}
$$

where $\mathcal{L}_{j} \stackrel{\text { def }}{=} \widetilde{\bar{u}_{j}} \bar{\theta}-\tilde{\bar{u}}_{j} \tilde{\bar{\theta}}$ is a resolved heat flux vector directly computable in the simulation. By substituting the grid and test-grid level SGS HF models into the vector identity and assuming that $\tilde{h}_{j}=-\widetilde{C_{\theta P} b_{j}^{P}} \approx-C_{\theta P} \tilde{b}_{j}^{P}$, a residual vector that accounts for the difference between the left-hand-side (LHS) and right-hand-side (RHS) of the 
vector identity emerges:

$$
\mathcal{E}_{j}=\mathcal{L}_{j}+C_{\theta P}\left(a_{j}^{P}-\tilde{b}_{j}^{P}\right)=\mathcal{L}_{j}+C_{\theta P} P_{j}
$$

where $P_{j} \stackrel{\text { def }}{=} a_{j}^{P}-\tilde{b}_{j}^{P}$ is a differential vector. The model coefficient $C_{\theta P}$ can be obtained by minimizing the norm of the residual vector (i.e., $\mathcal{E} \stackrel{\text { def }}{=} \mathcal{E}_{j} \mathcal{E}_{j}$ ) using the least squares method, as:

$$
C_{\theta P}=-\frac{\mathcal{L}_{j} P_{j}}{P_{j} P_{j}}
$$

\subsubsection{SGS Heat Flux Model 2 (DFLTDM)}

In the DFLTDM proposed by Wang et al. [61], the SGS heat flux is modelled as

$$
h_{j}=-D_{j k}^{L} \frac{\partial \bar{\theta}}{\partial x_{k}}=-C_{\theta P} \bar{\Delta}^{2}|\bar{S}| \frac{\partial \bar{\theta}}{\partial x_{j}}-C_{\theta G} \bar{\Delta}^{2} \bar{S}_{j k} \frac{\partial \bar{\theta}}{\partial x_{k}}
$$

where the tensor diffusivity is a full linear function of $\bar{S}_{i j}$, i.e.

$$
D_{j k}^{L}=C_{\theta P} \bar{\Delta}^{2}|\bar{S}| \delta_{j k}+C_{\theta G} \bar{\Delta}^{2} \bar{S}_{j k}
$$

The first term on the RHS of Eqs. (2.37) and (2.38) corresponds to the classical DEDM of Moin et al. [15], which is a function of $\bar{S}_{i j}$ of the zeroth-order, and the second term corresponds to the DHLTDM of Peng and Davidson [22], which is a homogeneous linear function of $\bar{S}_{i j}$ of the first-order. Therefore, the constitutive relation of the DFLTDM is based on a linear combination of these two conventional models.

For the DFLTDM, the grid and test-grid level SGS HF vectors can be expressed as $h_{j}=-C_{\theta P} b_{j}^{P}-C_{\theta G} b_{j}^{G}$ and $H_{j}=-C_{\theta P} a_{j}^{P}-C_{\theta G} a_{j}^{G}$, respectively. Here, $b_{j}^{G} \stackrel{\text { def }}{=} \bar{\Delta}^{2} \bar{S}_{j k} \frac{\partial \bar{\theta}}{\partial x_{k}}$, $a_{j}^{G} \stackrel{\text { def }}{=} \tilde{\bar{\Delta}}^{2} \tilde{\bar{S}}_{j k} \frac{\partial \tilde{\bar{\theta}}}{\partial x_{k}}$ are two base vector functions in analogy to the definitions of $b_{j}^{P}$ and $a_{j}^{P}$, respectively. Following a similar approach to derive the dynamic coefficient for the DEDM, the two dynamic model coefficients $C_{\theta P}$ and $C_{\theta G}$ for the DFLTDM can 
be obtained using the least squares method [9]:

$$
\left[\begin{array}{cc}
P_{j} P_{j} & P_{j} G_{j} \\
G_{j} P_{j} & G_{j} G_{j}
\end{array}\right] \cdot\left[\begin{array}{c}
C_{\theta P} \\
C_{\theta G}
\end{array}\right]=-\left[\begin{array}{c}
\mathcal{L}_{j} P_{j} \\
\mathcal{L}_{j} G_{j}
\end{array}\right],
$$

where $P_{j}$ and $G_{j} \stackrel{\text { def }}{=} a_{j}^{G}-\tilde{b}_{j}^{G}$ are differential vectors. We will primarily use the DFLTDM for closure of the thermal energy equation throughout this paper. However, on some special occasions (when we study the budget of the wall-normal heat fluxes), the DEDM of Moin et al. [15] will also be used in order to compare the predictive performance of these two SGS HF models.

\subsection{Numerical Algorithm}

In this thesis, the fractional time-step technique commonly used for performing DNS and LES proposed by Kim and Moin [62] is used to develop the flow solver. The filtered Navier-Stokes equations are solved using two steps: first, an estimated velocity field is obtained by solving the governing equation without updating the pressure field; and then a Poisson equation for pressure correction derived from the filtered continuity equation is solved and the estimated velocity field is further corrected to satisfy mass conservation. The filtered Navier-Stokes equation (2.10) can be written as

$$
\frac{\partial \bar{u}_{i}}{\partial t}=-\frac{1}{\rho} \frac{\partial \bar{p}}{\partial x_{i}}-\mathcal{H}_{i}+\nu \frac{\partial^{2} \bar{u}_{i}}{\partial x_{j} \partial x_{j}}+\mathcal{F}_{i}
$$

where $\mathcal{H}_{i}$ and $\mathcal{F}_{i}$ are the nonlinear and the Coriolis force terms, which are expressed as

$$
\mathcal{H}_{i}=\frac{\partial}{\partial x_{j}}\left(\bar{u}_{i} \bar{u}_{j}\right)+\frac{\partial \tau_{i j}}{\partial x_{j}}
$$

and

$$
\mathcal{F}_{i}=2 \epsilon_{i j 1} \Omega \bar{u}_{j}
$$

respectively. The velocity field after a time step $\Delta t$ is updated based on applying a typical time advancement method which uses a second-order Adams-Bashforth 
scheme for the nonlinear terms, a Crank-Nicolson scheme for the viscous stress terms, and an explicit scheme for the Coriolis force term

$$
\frac{\bar{u}_{i}^{(*)}-\bar{u}_{i}^{(n)}}{\Delta t}=-\frac{1}{2}\left[3 \mathcal{H}_{i}^{(n)}-\mathcal{H}_{i}^{(n-1)}\right]+\frac{\nu}{2}\left[\frac{\partial^{2} \bar{u}_{i}^{(*)}}{\partial x_{j} \partial x_{j}}+\frac{\partial^{2} \bar{u}_{i}^{(n)}}{\partial x_{j} \partial x_{j}}\right]+\mathcal{F}_{i}^{(n)}
$$

and

$$
\bar{u}_{i}^{(n+1)}=\bar{u}_{i}^{(*)}-\frac{\Delta t}{\rho} \frac{\partial \bar{p}^{(n+1)}}{\partial x_{i}},
$$

where superscript $(n)$ indicates the current time step and superscript $\left(^{*}\right)$ indicates an intermediate step between the current and next time steps (denoted using superscript $(n+1))$.

In order to solve Eqs. (2.43) and (2.44), the 3-D governing equation is discretized using a finite volume (FV) method based on a co-llocated grid system. In a co-llocated grid system, the information on both the velocity and the pressure field is stored at cell centers, and the velocities at the interfaces of a control volume are approximated by an interpolation of the neighboring nodal velocities stored at the cell centers. A second-order central difference scheme was applied for the spatial discretization of the Eq. (2.43) as it represents a good compromise between accuracy, simplicity and efficiency [8]. Then $\bar{p}^{(n+1)}$ is obtained by updating the old pressure $\bar{p}^{(n)}$ with an additional correcting pressure $\bar{p}^{\prime}$ as

$$
\bar{p}^{(n+1)}=\bar{p}^{(n)}+\bar{p}^{\prime}
$$

The unknown $\bar{p}^{\prime}$ is derived from the filtered continuity equation (2.9) to satisfy mass conservation. By applying the FV method based on the collocated grid system, the continuity equation can be discretized as shown in Fig. 2.1, viz.

$$
\int_{b}^{f} \int_{s}^{n} \int_{w}^{e}\left(\frac{\partial \bar{u}_{1}}{\partial x_{1}}+\frac{\partial \bar{u}_{2}}{\partial x_{2}}+\frac{\partial \bar{u}_{3}}{\partial x_{3}}\right) d x_{1} d x_{2} d x_{3}=0
$$

By integrating the above equation, we obtain: 


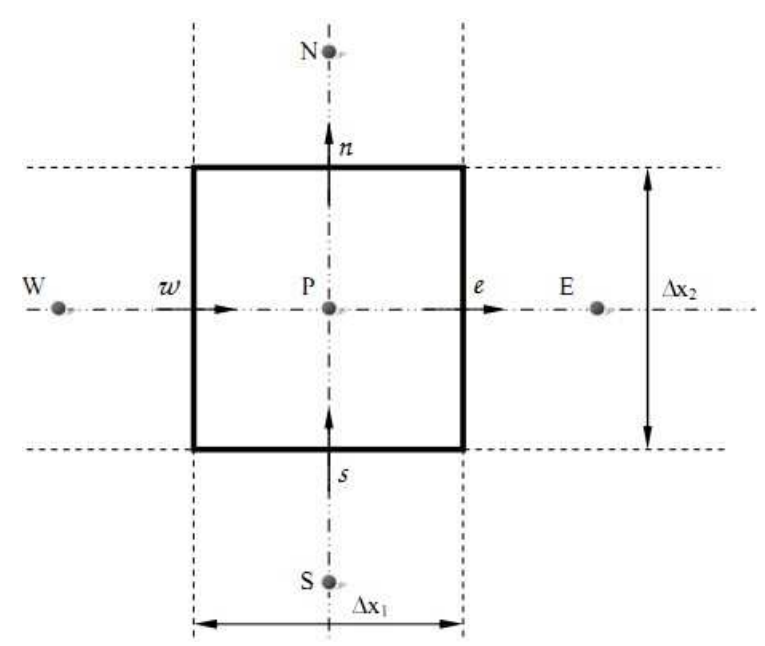

FIGURE 2.1: A two-dimensional sketch of a typical control volume for a co-llocated grid system.

$$
\left(\bar{u}_{e}-\bar{u}_{w}\right) A_{1}+\left(\bar{u}_{n}-\bar{u}_{s}\right) A_{2}+\left(\bar{u}_{f}-\bar{u}_{b}\right) A_{3}=0
$$

where subscripts 'e', 'w', 'n', 's', 'f' and 'b' represent 'east', 'west', 'north', 'south', 'front' and 'back' faces of the control volume, respectively, and $A_{1}=\Delta x_{2} \cdot \Delta x_{3}$, $A_{2}=\Delta x_{1} \cdot \Delta x_{3}$ and $A_{3}=\Delta x_{1} \cdot \Delta x_{2}$ are the cross-sectional areas of the control volume perpendicular to the w-e, s-n and b-f directions, respectively. The face velocities in Eq. (2.47) are approximated by interpolation of neighboring node velocities. For example, the velocity at the east face is calculated as:

$$
\bar{u}_{e}=\frac{\bar{u}_{P}^{(n+1)}+\bar{u}_{E}^{(n+1)}}{2}
$$

where the uppercase subscripts denote the neighboring nodes as shown in Fig. 2.1. By substituting the Eq. (2.44) into the above equation, the nonlinear momentum interpolation scheme (originally proposed by Rhie and Chow [63]) for the face velocity is implemented, and we obtain:

$$
\begin{aligned}
\bar{u}_{e} & =\frac{1}{2}\left\{\left[\bar{u}_{P}^{(*)}-\left.\frac{\Delta t}{\rho} \frac{\partial \bar{p}^{(n+1)}}{\partial x}\right|_{P}\right]+\left[\bar{u}_{E}^{(*)}-\left.\frac{\Delta t}{\rho} \frac{\partial \bar{p}^{(n+1)}}{\partial x}\right|_{E}\right]\right\} \\
& =\frac{\bar{u}_{P}^{(*)}+\bar{u}_{E}^{(*)}}{2}-\frac{\Delta t}{2 \rho}\left[\left.\frac{\partial \bar{p}^{(n+1)}}{\partial x}\right|_{P}+\left.\frac{\partial \bar{p}^{(n+1)}}{\partial x}\right|_{E}\right] \\
& \approx \frac{\bar{u}_{P}^{(*)}+\bar{u}_{E}^{(*)}}{2}-\left.\frac{\Delta t}{\rho} \frac{\partial \bar{p}^{(n+1)}}{\partial x}\right|_{e} .
\end{aligned}
$$


Let

$$
\left.\frac{\partial \bar{p}^{(n+1)}}{\partial x}\right|_{e}=\frac{\bar{p}_{E}^{(n+1)}-\bar{p}_{P}^{(n+1)}}{\Delta x_{P E}},
$$

where $\Delta x_{P E}=x_{E}-x_{P}$. Using Eq. (2.50) and (2.45), Eq. (2.49) can be written as:

$$
\begin{aligned}
\bar{u}_{e} & =\frac{\bar{u}_{P}^{(*)}+\bar{u}_{E}^{(*)}}{2}-\frac{\Delta t}{\rho}\left[\frac{\bar{p}_{E}^{(n+1)}-\bar{p}_{P}^{(n+1)}}{\Delta x_{P E}}\right] \\
& =\frac{\bar{u}_{P}^{(*)}+\bar{u}_{E}^{(*)}}{2}-\frac{\Delta t}{\rho}\left[\frac{\left(\bar{p}_{E}^{(n)}+\bar{p}_{E}^{\prime}\right)\left(\bar{p}_{P}^{(n)}+\bar{p}_{P}^{\prime}\right)}{\Delta x_{P E}}\right] \\
& =\frac{\bar{u}_{P}^{(*)}+\bar{u}_{E}^{(*)}}{2}-\frac{\Delta t}{\rho}\left[\frac{\bar{p}_{E}^{(n)}-\bar{p}_{P}^{(n)}}{\Delta x_{P E}}\right]-\frac{\Delta t}{\rho}\left[\frac{\bar{p}_{E}^{\prime}-\bar{p}_{P}^{\prime}}{\Delta x_{P E}}\right] .
\end{aligned}
$$

Let

$$
\bar{u}_{e}^{*}=\frac{\bar{u}_{P}^{*}+\bar{u}_{E}^{*}}{2}-\frac{\Delta t}{\rho}\left[\frac{\bar{p}_{E}^{(n)}-\bar{p}_{P}^{(n)}}{\Delta x_{P E}}\right] .
$$

Eq. (2.52) becomes:

$$
\bar{u}_{e}=\bar{u}_{e}^{*}-\frac{\Delta t}{\rho}\left[\frac{\bar{p}_{E}^{\prime}-\bar{p}_{P}^{\prime}}{\Delta x_{P E}}\right] .
$$

Substituting Eq. (2.53) into Eq. (2.47), the following discrete equation for the correcting pressure is obtained:

$$
a_{P} \bar{p}_{P}^{\prime}=\sum a_{N P} \bar{p}_{N P}^{\prime}+b
$$

where subscript 'NP' represents the neighboring nodes; and ' $a$ ' and 'b' denote the coefficients and source term, respectively, all of which are:

$$
\begin{aligned}
& a_{E}=\frac{A_{1} \Delta t}{\Delta x_{P E}}, \quad a_{W}=\frac{A_{1} \Delta t}{\Delta x_{W P}}, \quad a_{S}=\frac{A_{2} \Delta t}{\Delta x_{P S}}, \\
& a_{N}=\frac{A_{2} \Delta t}{\Delta x_{N P}}, \quad a_{F}=\frac{A_{3} \Delta t}{\Delta x_{P F}}, \quad a_{B}=\frac{A_{3} \Delta t}{\Delta x_{B P}}, \\
& b=-\rho\left[A_{1}\left(\bar{u}_{e}^{*}-\bar{u}_{w}^{*}\right)+A_{2}\left(\bar{u}_{n}^{*}-\bar{u}_{s}^{*}\right)+A_{3}\left(\bar{u}_{f}^{*}-\bar{u}_{b}^{*}\right)\right], \\
& a_{P}=\sum a_{N P} .
\end{aligned}
$$

Note that in the above scheme, there are two steps for calculating the face velocity: first, the neighboring velocities and the pressure gradient $-\partial \bar{p}^{(n)} / \partial x_{i}$ at the interface (see Eq. (2.52)) are used to estimate the face velocity, and then by using the pressure 


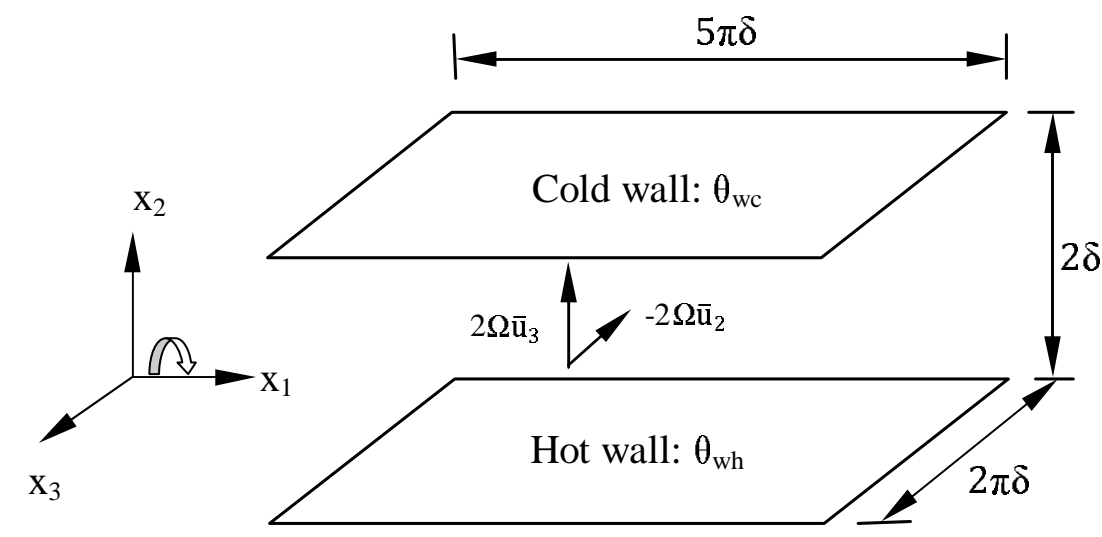

FIGURE 2.2: Computational domain for a heated streamwise rotating channel flow. The two Coriolis forces are: $-2 \Omega \bar{u}_{2}$ and $2 \Omega \bar{u}_{3}$.

correction (see Eq. (2.53)), it is corrected to satisfy mass conservation. Instead of deriving a pressure Poisson equation for solving $\bar{p}^{(n+1)}$, this approach leads to a pressure correction Poisson equation (2.54) for solving $\bar{p}^{\prime}$. The convergence creterion for solving the $\bar{p}^{\prime}$ equation is $\left|r^{(n)} / r^{(n-1)}\right|<0.01$, where $r^{(n)}=\left(\sum a_{N P} \bar{p}_{N P}^{\prime}+b-a_{P} \bar{p}_{P}^{\prime}\right)^{(n)}$. A multigrid method based on a special control scheme introduced by Yin [64] is adopted in this research to effectively solve this equation. A fourth-order Runge-Kutta method was used to advance the temperature field over a single time step in order to solve the filtered thermal energy equation (2.11).

\subsection{Test Case and Computational Domain}

The computational domain of the heated rotating plane channel and coordinate system used in the numerical simulation is shown in Figure 2.2. The system of the plane channel rotates with a constant positive angular velocity $\Omega$, parallel to the $x_{1}$ direction. Two Reynolds numbers are used to characterize the flow respectively, which are $R e_{\tau} \stackrel{\text { def }}{=} u_{\tau} \delta / \nu=150$ and 300. Here, $u_{\tau} \stackrel{\text { def }}{=} \sqrt{\tau_{w} / \rho}$ represents the wall friction velocity and $\nu$ is the kinematic viscosity of the fluid. The Prandtl number of the fluid is set to $\operatorname{Pr}=0.71$. In order to examine the effects of rotation on the fluid flow, a wide range of rotation numbers $\left(R O_{\tau} \stackrel{\text { def }}{=} 2 \Omega \delta / u_{\tau}\right)$ ranging 0 to 15 are tested. The dimensions of the computational domain are $L_{1} \times L_{2} \times L_{3}=5 \pi \delta \times 2 \delta \times 2 \pi \delta$ in the 
streamwise $\left(x_{1}\right)$, wall-normal $\left(x_{2}\right)$ and spanwise $\left(x_{3}\right)$ directions, respectively. A grid system with $48 \times 32 \times 48$ control volumes is used to discretize the computational domain for $R e_{\tau}=150$, and a finer grid system with $64 \times 64 \times 64$ control volumes is used for $R e_{\tau}=300$. The grid is uniform in the streamwise and spanwise directions, and stretched in the wall-normal direction using a hyperbolic-tangent function in order to provide a greater resolution in the near-wall region.

\subsection{Boundary Conditions}

In this research, non-slip and impermeable boundary conditions are imposed on the velocity components at the walls. Periodic boundary conditions are employed in the streamwise and spanwise directions since the flow and temperature fields are assumed to be statistically homogeneous in both these directions. The temperature field has been non-dimensionalized by the temperature difference (i.e., $\Delta \theta=\theta_{w h}-\theta_{w c}$, see Fig. 2.2) between the two plates. The initial temperature field is assumed to be linearly distributed in the wall-normal direction between the two walls. The initial velocity profile is assumed to be parabolic characteristic of a Poiseuille type laminar flow. The maximum Courant-Friedrichs-Lewy (CFL) number used in the simulations is limited to 0.35 . Statistics of various flow variables are calculated based on 60,000 time steps after both the fluid and thermal fields have become turbulent and fullydeveloped.

In presentation of the results, a superscript ' + ' denotes the quantities nondimensionalized using the friction velocity $u_{\tau} \stackrel{\text { def }}{=} \sqrt{\tau_{w} / \rho}$ and friction temperature $\theta_{\tau} \stackrel{\text { def }}{=} q_{w} /\left(\rho c_{P} u_{\tau}\right)$. Here, $q_{w}$ represents the wall heat flux and $c_{P}$ is the specific heat at constant pressure. 


\section{Chapter 3}

\section{Statistics of the Turbulent Thermal Flow Fields}

In this chapter, the characteristics of the resolved velocity and temperature fields in terms of their mean values and fluctuations are thoroughly analyzed. In order to validate the numerical approach, the LES results obtained from the simulations are compared with the reported DNS data. For the test cases of $R e_{\tau}=150$, the DNS data of El-Samni and Kasagi [65] (designated as EK-2000) for a heated rotating channel flow $\left(R o_{\tau}=15.0\right)$ are compared with the LES results under the same operating conditions, and two sets of DNS data on non-rotating channel flows $\left(R o_{\tau}=0\right)$ obtained by Kuroda et al. [66] (designated as KKN-1995) and Iwamoto et al. [67] (designated as ISK-2002) are also used in our comparative study. For the test cases of $R e_{\tau}=300$, the LES results for a heated rotating channel flow $\left(R o_{\tau}=2.5\right)$ are compared with the DNS data of Wu and Kasagi [53] (designed as WK-2004), and the results of the non-rotating case $\left(R o_{\tau}=0\right)$ are compared with the DNS data of Iwamoto et al. [68] (designated as ISK-2004).

\subsection{Resolved Mean Velocity and Temperature}

Figures 3.1, 3.2 and 3.3 show the profiles of the mean resolved streamwise velocity $\left\langle\bar{u}_{1}\right\rangle$, spanwise velocity $\left\langle\bar{u}_{3}\right\rangle$ and temperature $\langle\bar{\theta}\rangle$ in the wall-normal direction across the channel, respectively. The LES results are validated with the DNS data at the two 


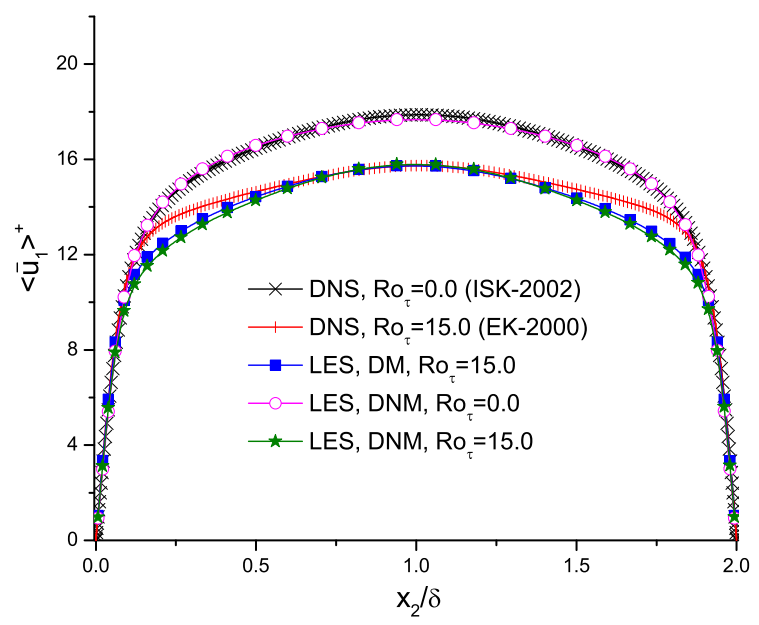

(a) $R e_{\tau}=150$

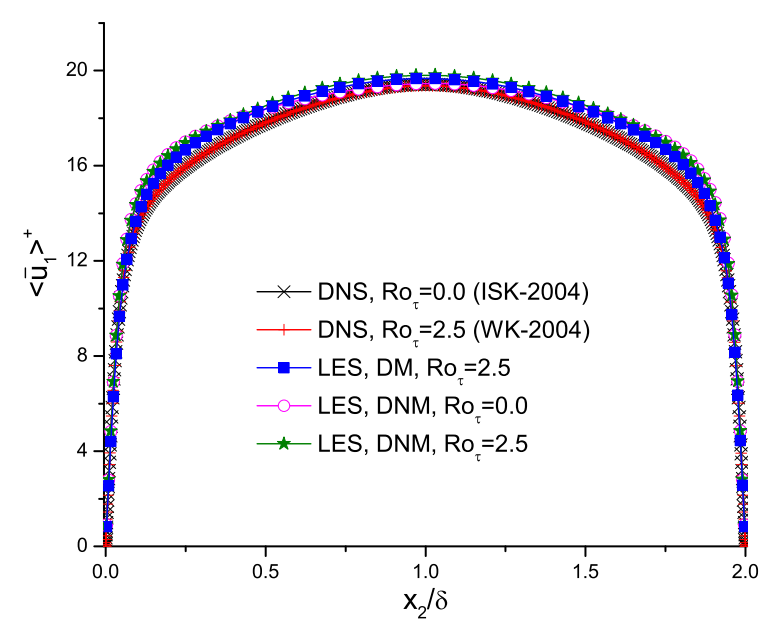

(b) $R e_{\tau}=300$

FIGURE 3.1: Profiles of the resolved mean streamwise velocity for $R o_{\tau}=0$ and 15.0 at $R e_{\tau}=150, R o_{\tau}=0$ and 2.5 at $R e_{\tau}=300$ (predicted in conjunction with the DFLTDM).

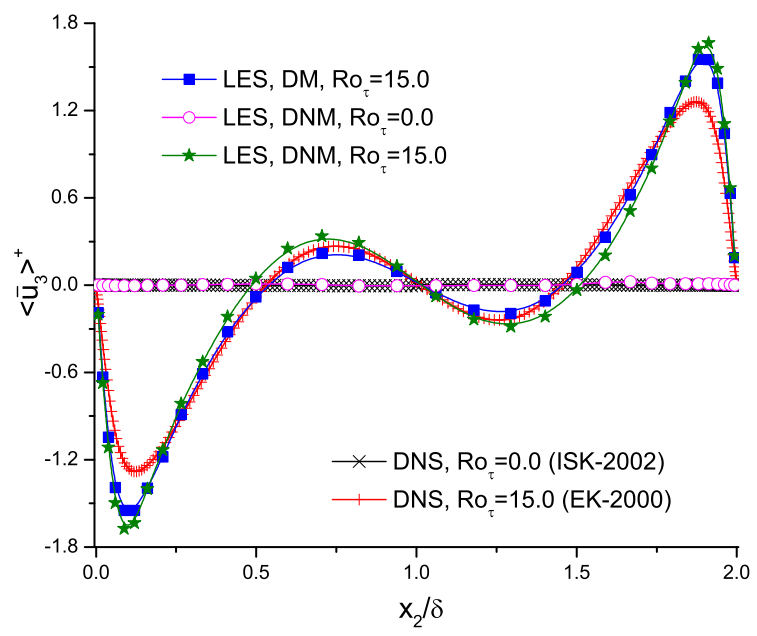

(a) $R e_{\tau}=150$

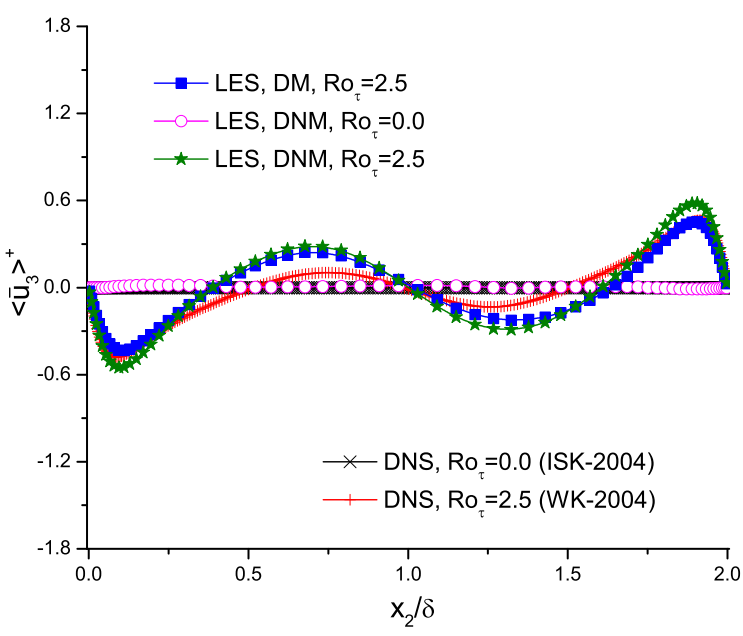

(b) $R e_{\tau}=300$

FIGURE 3.2: Profiles of the resolved mean spanwise velocity for $R o_{\tau}=0$ and 15.0 at $R e_{\tau}=150, R o_{\tau}=0$ and 2.5 at $R e_{\tau}=300$ (predicted in conjunction with the DFLTDM).

Reynolds numbers for $R e_{\tau}=150$ and 300. As shown in these figures, the performance of the DNM and DM is similar when both SGS stress models are tested in conjunction with the same SGS HF model DFLTDM. As shown in Fig. 3.1, the LES result of the mean resolved streamwise velocity is in excellent agreement with the DNS data. However, as shown in Figs. 3.2(a) and (b), the mean resolved spanwise velocity in the 


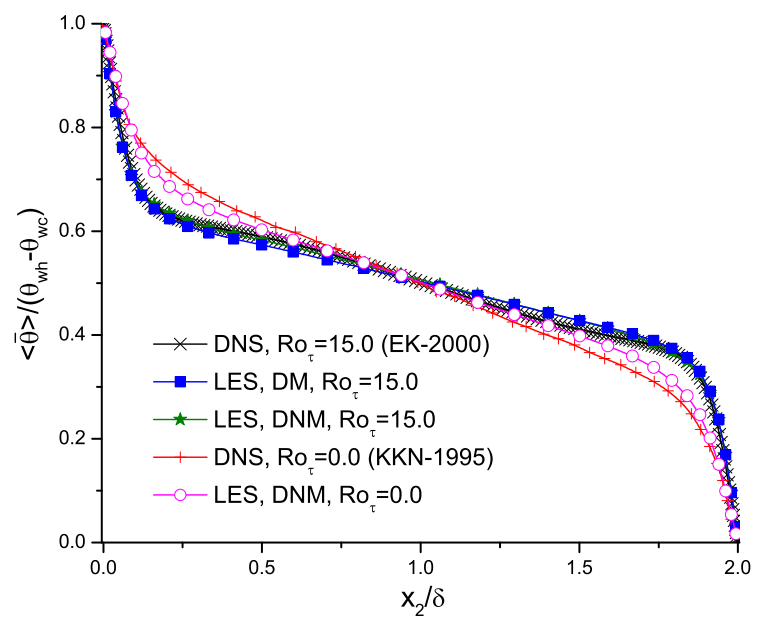

(a) $R e_{\tau}=150$

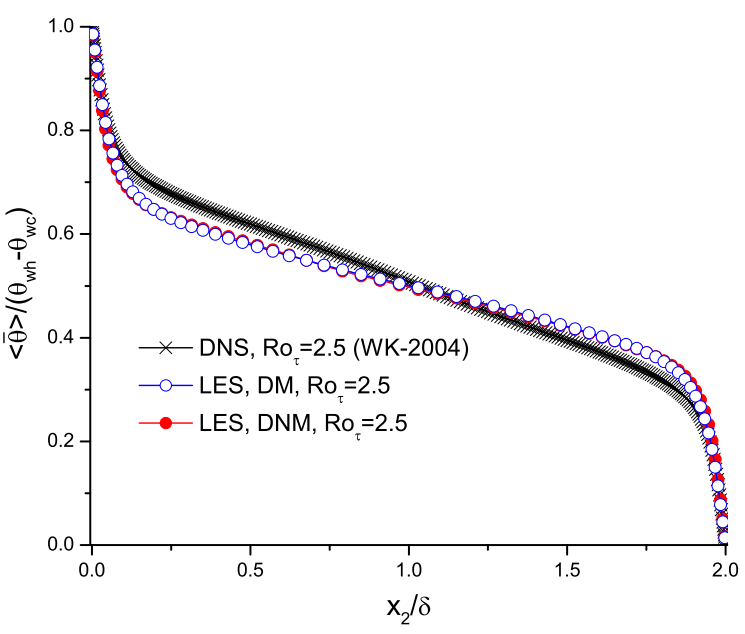

(b) $R e_{\tau}=300$

FIGURE 3.3: Profiles of the resolved mean temperature for $R o_{\tau}=0$ and 15.0 at $R e_{\tau}=150, R o_{\tau}=2.5$ at $R e_{\tau}=300$ (predicted in conjunction with the DFLTDM).

near wall region at $R e_{\tau}=150$ and near the central plane at $R e_{\tau}=300$ are slightly overpredicted by LES. In Fig. 3.3, at the rotating case, LES slightly underpredicts the resolved mean temperature.

In the spanwise direction, as evident in Fig. 3.2, in response to the Coriolis and centrifugal forces intrinsic to a rotating flow, a complex secondary flow pattern is formed. The profile of the mean resolved spanwise velocity is skew-symmetric about the central plane $\left(x_{2} / \delta=1\right)$ of the domain, and four distinct layers of opposite motion are observed between the two planes. This interesting pattern in the mean resolved velocity profile $\left\langle\bar{u}_{3}\right\rangle$ is characteristic of a streamwise rotating channel flow, which is consistent with the observation of El-Samni and Kasagi [65] and Wu and Kasagi [53] based on their DNS study.

The effects of the system rotation at two different Reynolds numbers on the resolved mean streamwise and spanwise velocities and temperature are examined by comparing numerical results obtained at a variety of rotation numbers. Figure 3.4 displays the influence of system rotation on the resolved mean streamwise velocity $\left\langle\bar{u}_{1}\right\rangle$. As shown in Fig. 3.4(a), at $R e_{\tau}=150$, the magnitude of streamwise velocity decreases monotonically as the rotation number increases, which corresponds to the trend of a decreasing streamwise mass flow rate. However, in Fig. 3.4(b), no obvious 


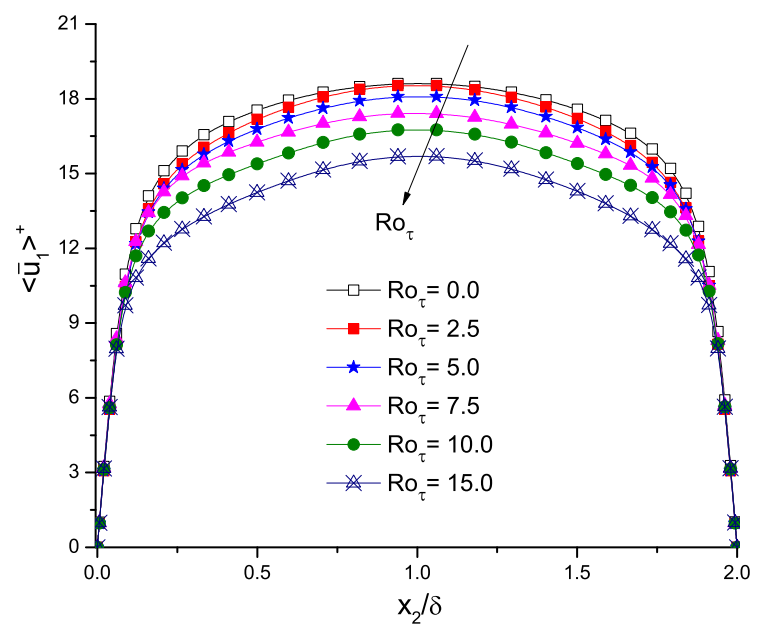

(a) $R e_{\tau}=150$

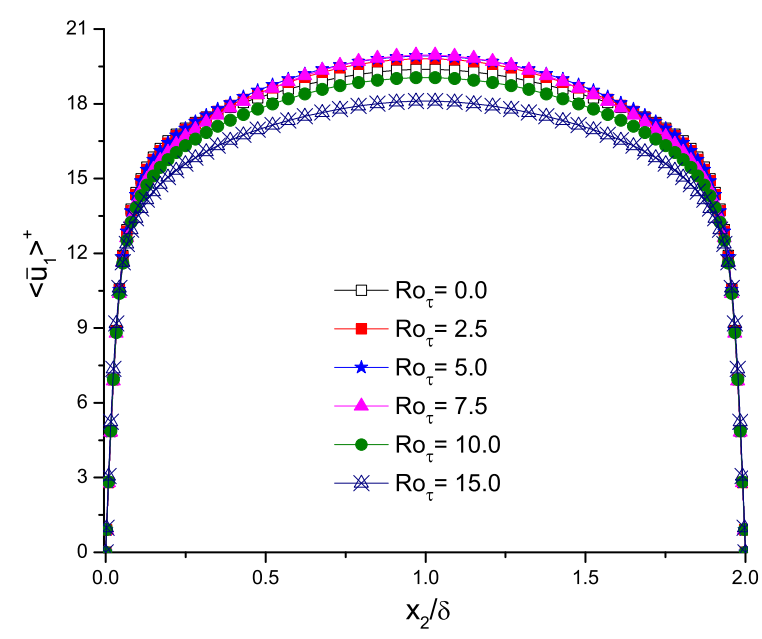

(b) $R e_{\tau}=300$

FIGURE 3.4: Profiles of the resolved mean streamwise velocity at various rotation numbers (predicted using the DNM and DFLTDM).

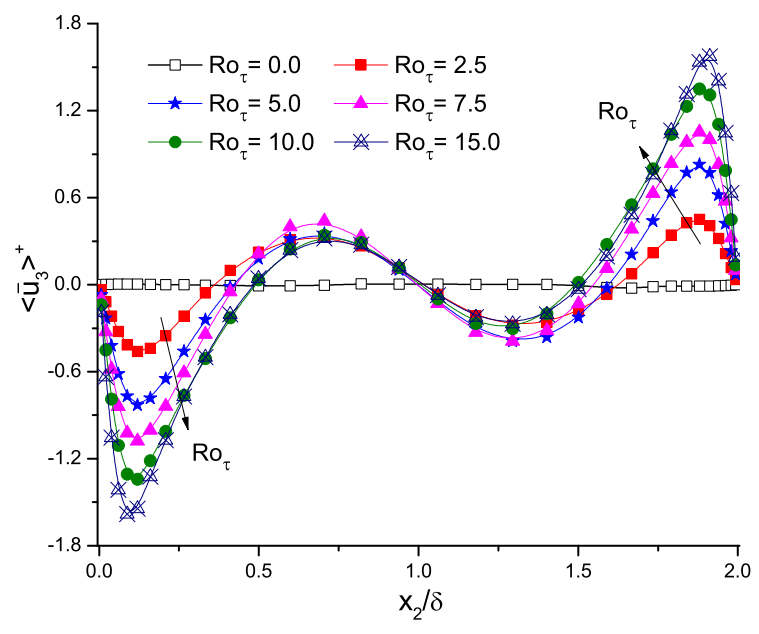

(a) $R e_{\tau}=150$

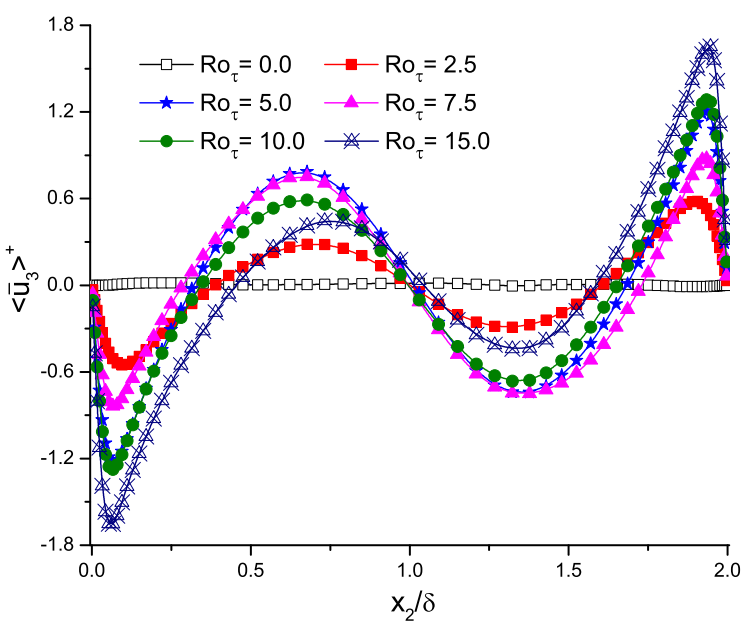

(b) $R e_{\tau}=300$

FIGURE 3.5: Profiles of the resolved mean spanwise velocity at various rotation numbers (predicted using the DNM and DFLTDM).

discrepancies are observed at relative low rotation numbers until $R o_{\tau}$ reaches to 10 at $R e_{\tau}=300$. This phenomenon indicates that the reduction of mass flow rate in the streamwise direction due to the streamwise system rotation becomes less sensitive at a higher Reynolds number.

The large spanwise skew-symmetric secondary flow previously observed in Fig. 3.2 is further examined in Fig. 3.5 based on tests of a number of rotation numbers. As 


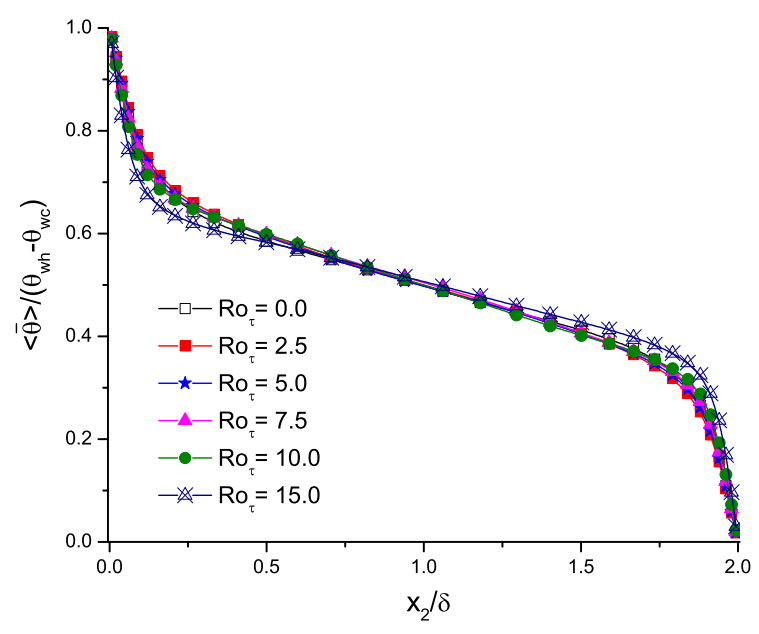

(a) $R e_{\tau}=150$

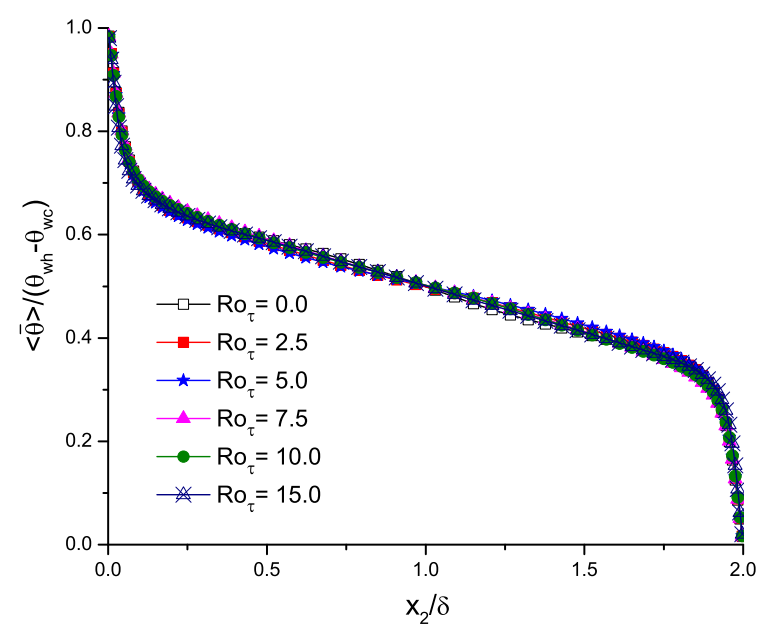

(b) $R e_{\tau}=300$

FIGURE 3.6: Profiles of the resolved mean temperature at various rotation numbers (predicted using the DNM and DFLTDM).

shown in Fig. 3.5, in response to the Coriolis force, a complex secondary flow pattern is formed in the spanwise direction. As shown in both Figs. 3.5(a) and (b), there are four peaks in the mean resolved spanwise velocity profile (corresponding to the four distinct layers of opposite motion), two in the near-wall regions and two close to the central plane $\left(x_{2} / \delta=1\right)$ of the domain. As shown in Fig. 3.5(a), at $R e_{\tau}=150$, the absolute value of the velocity gradient of the secondary flow increases monotonically as the rotation number increases. However, at $R e_{\tau}=300$, only the peak magnitude in the near-wall region increases monotonically as the rotation number increases; whereas, the peak magnitude near the central plane increases with the rotation number only if the rotation number is smaller than $R o_{\tau}=7.5$. Once the $R o_{\tau}$ is large than 7.5 , the rotation effect on the magnitude of $\left\langle\bar{u}_{3}\right\rangle$ reverses near the central plane. The physical mechanism underlying this interesting dynamical feature for $R e_{\tau}=300$ is due to fact that the formation of these four distinct layers of secondary flow motion depends upon not only the Coriolis force but also the centrifugal force. The magnitude of the local centrifugal force relates to both the rotation number and the distance from the local position to the rotation axis. Furthermore, the spanwise component of the centrifugal force has the same direction of the secondary flow in near-wall region, but the opposite direction with the secondary flow near the central plane. For this 
reason, as the rotation number increases, the secondary flow near the wall is enhanced, whereas the secondary flow near the central line is damped.

In Fig. 3.6, in comparison with the non-rotating case, the gradient of the temperature profile for the rotating case increases slightly across the channel at $R e_{\tau}=150$, however, no obvious variation is observed at $R e_{\tau}=300$. This indicates that the system rotation in the streamwise direction does not have a significant impact on the resolved mean temperature distribution in the wall-normal direction, especially at a higher Reynolds number.

Figures 3.7 and 3.8 display the profiles of the mean resolved streamwise velocity and temperature using wall coordinates, respectively. The results of $R e_{\tau}=150$ for $R o_{\tau}=0.0$ and $R o_{\tau}=15.0$, and those of $R e_{\tau}=300$ for $R o_{\tau}=0$ and $R o_{\tau}=2.5$ are compared against reported relevent DNS data in these figures. For the mean resolved streamwise velocity and temperature profiles, both the DNM and DM yield results that are consistent with the DNS data. It is shown in Figs. 3.7 and 3.8 that at nonrotating case, the profiles are in agreement with the classical log laws of the wall for a smooth zero-pressure-gradient $(\mathrm{ZPG})$ boundary layer, i.e. $\left\langle\bar{u}_{1}\right\rangle^{+}=2.5 \ln \left(x_{2}^{+}\right)+5.5$ and $\langle\bar{\theta}\rangle^{+}=2.195 \ln \left(x_{2}^{+}\right)+13.2 \operatorname{Pr}-5.66$ for $x_{2}^{+}>30$. It needs to be indicated that at $R e_{\tau}=150$, the DNM and DM give similar prediction results (for $R o_{\tau}=15.0$ ) for the mean temperature. However, at $R e_{\tau}=300$, in comparison with the results obtained based on the DNM (for $R o_{\tau}=2.5$ ), the DM slightly overpredicts the mean temperature in Fig. 3.8(b). The fact that the difference in model predictions is the minimum in Fig. 3.3(b) but enlarged in Fig. 3.8(b) is related to the different methods of non-dimensionalization adopted. The wall coordinates used in Fig. 3.8(b) has an advantage in demonstrating the predictive accuracy of the model in terms of the wall friction temperature and velocity, especially at a higher Reynolds number.

The resolved mean streamwise velocity and temperature profiles based on the wall coordinates at various rotation numbers (ranging from $R o_{\tau}=0$ to 15) are compared in Figs. 3.9 and 3.10, respectively. As discussed previously, in response to the streamwise system rotation, organized large secondary flows appear in the cross- 


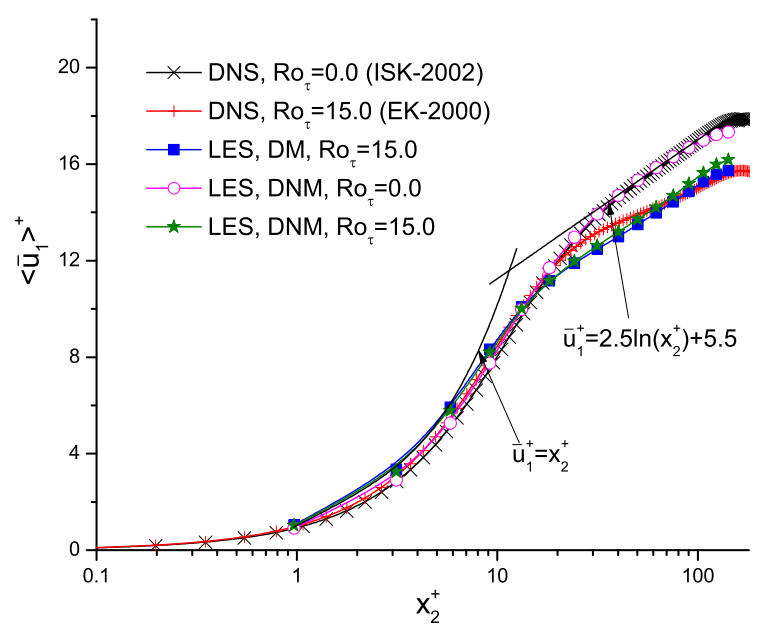

(a) $R e_{\tau}=150$

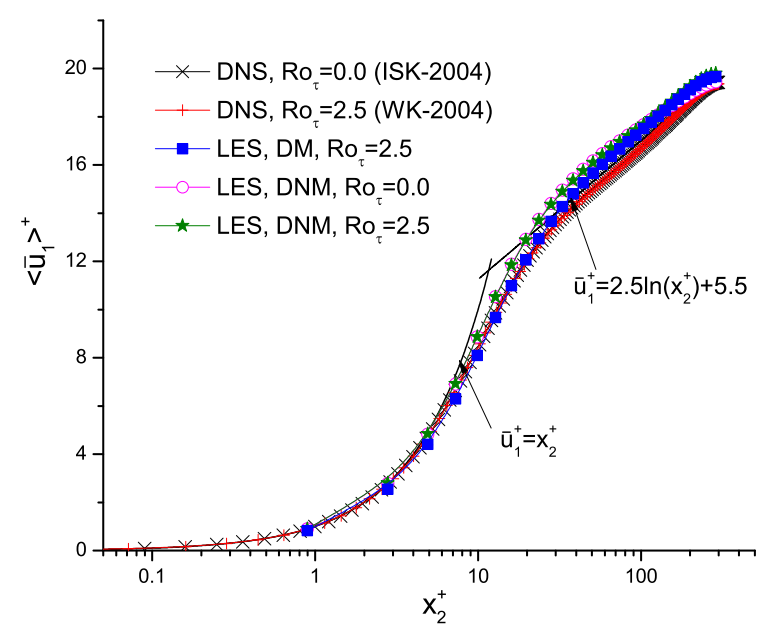

(b) $R e_{\tau}=300$

FIGURE 3.7: Profiles of the resolved mean streamwise velocity for $R o_{\tau}=0$ and 15.0 at $R e_{\tau}=150, R o_{\tau}=0$ and 2.5 at $R e_{\tau}=300$, displayed using wall coordinates (predicted in conjunction with the DFLTDM).

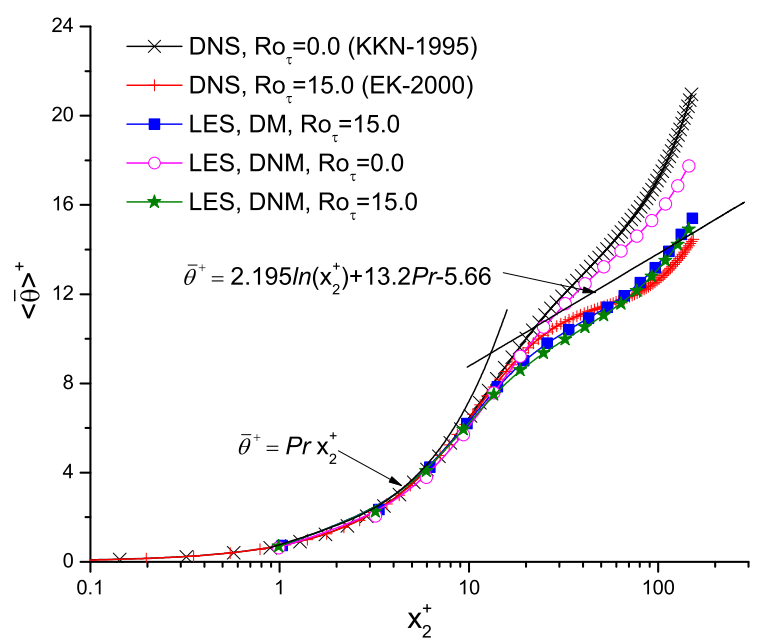

(a) $R e_{\tau}=150$

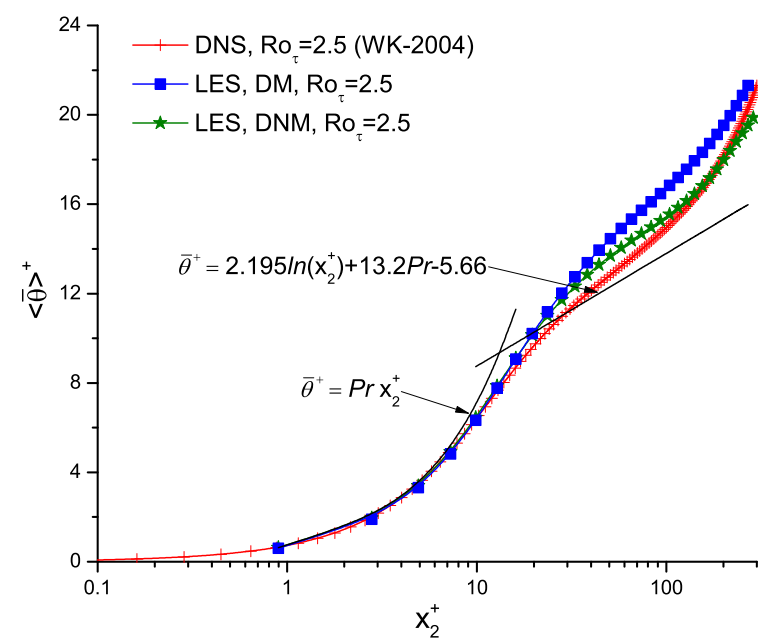

(b) $R e_{\tau}=300$

FIGURE 3.8: Profiles of the resolved mean temperature for $R o_{\tau}=0$ and 15.0 at $R e_{\tau}=150, R o_{\tau}=2.5$ at $R e_{\tau}=300$, displayed using wall coordinates (predicted in conjunction with the DFLTDM).

stream direction and four distinct layers of opposite motion are formed in the $\left(x_{2}\right.$, $x_{3}$ )-plane. However, the flow is the least sensitive to the imposed system rotation in the streamwise direction especially at a higher Reynolds number $\left(R e_{\tau}=300\right)$. For this reason, the mean streamwise velocity profiles shown in Fig. 3.9(b) do not deviate obviously from the classical log law. In contrast, as shown in Fig. 3.10(b), 


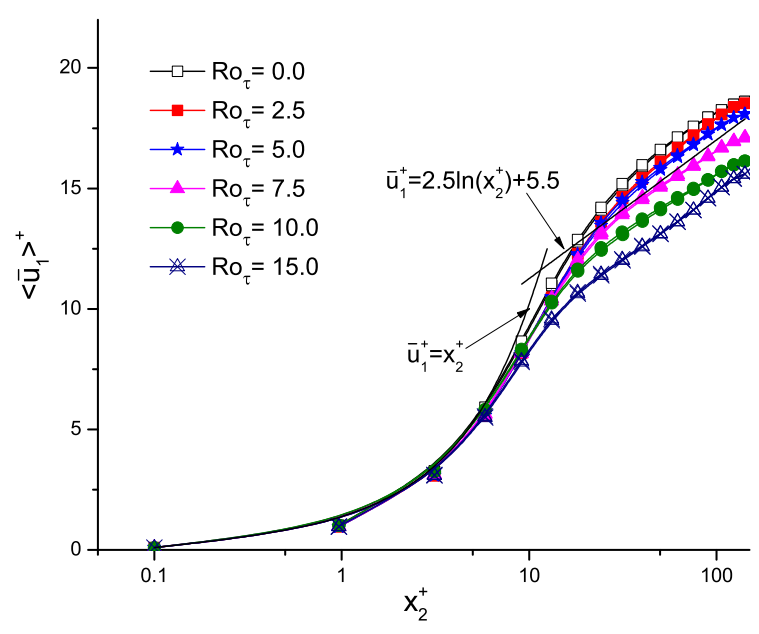

(a) $R e_{\tau}=150$

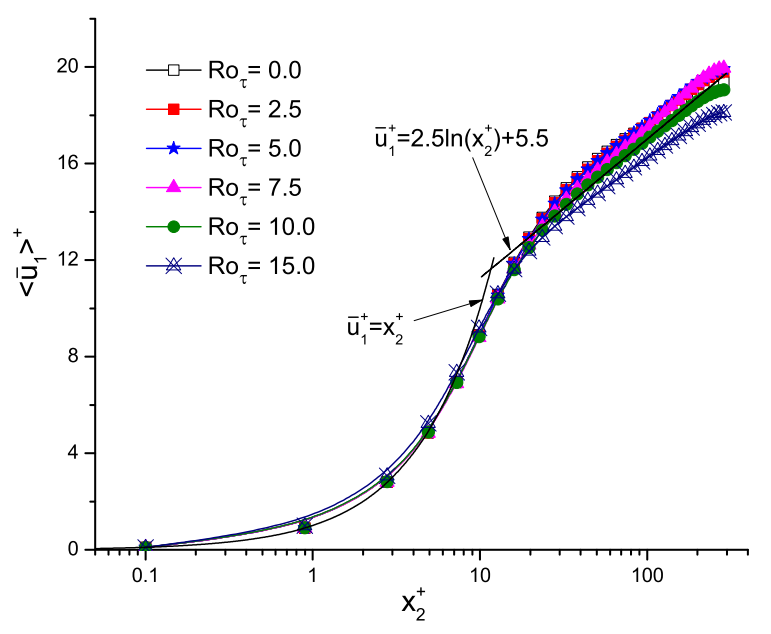

(b) $R e_{\tau}=300$

FIGURE 3.9: Profiles of the resolved mean streamwise velocity at various rotation numbers, displayed using wall coordinates (predicted using the DNM and DFLTDM).

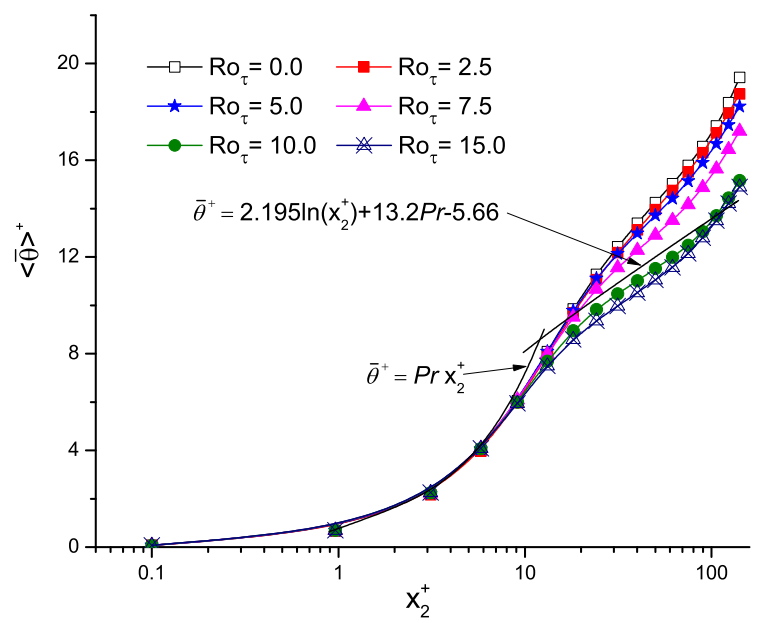

(a) $R e_{\tau}=150$

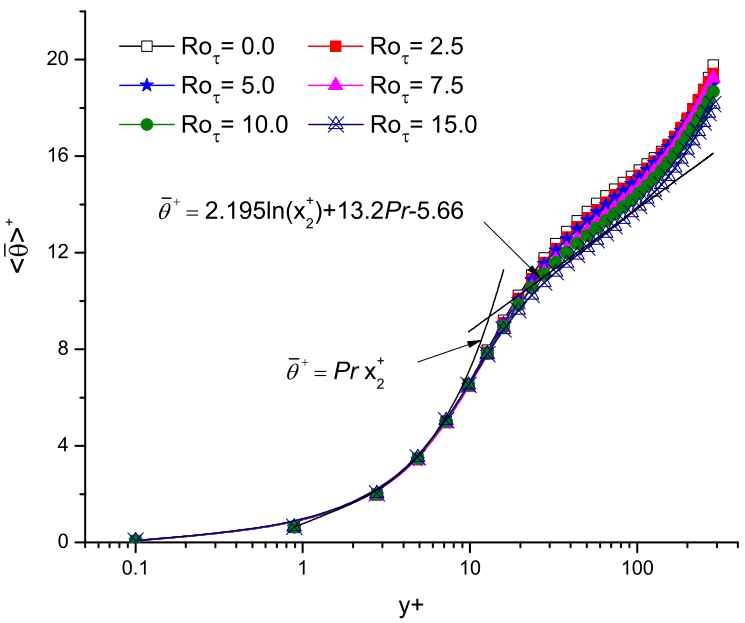

(b) $R e_{\tau}=300$

FIGURE 3.10: Profiles of the resolved mean temperature at various rotation numbers, displayed using wall coordinates (predicted using the DNM and DFLTDM).

the predicted mean temperature profiles for the rotating cases all deviate from the familiar log law (for a passive scalar in a ZPG wall shear layer). This physical feature of the mean temperature distribution is expected, as the transport of temperature (or, thermal energy) between two walls is dominated by convection and diffusion not only in the streamwise directly but also in the spanwise and wall-normal directions. Therefore, although the mean streamwise velocity profiles approximately follow the classical log law of velocity, the mean temperature profile deviate from the log law of 
scalar (owing to, e.g. the special secondary flow pattern in the $\left(x_{2}, x_{3}\right)$-plane). Also as demostrated previously, the flow is very sensitive to the imposed system rotation at $R e_{\tau}=150$. It is shown in Fig. 3.9(a) that the profiles of the resolved mean streamwise velocity shift downwards nonotonically and deviate obviously from the classical log law as the rotation number increases. This trend is preserved in the profile of the mean temperature profile shown in Fig. 3.10(a).

\subsection{Resolved Velocity and Temperature Fluctua- tions}

In order to further investigate the performance of different LES models under the same test condition, it is popular to directly compare the values of turbulent intensities obtained from LES with DNS approach. An instantaneous filtered quantity $\bar{\phi}$ can be decomposed into a time- and plane-averaged component and a residual component as

$$
\bar{\phi}=\langle\bar{\phi}\rangle+\bar{\phi}^{\prime \prime}
$$

Then the predicted resolved velocity and temperature fluctuations (or RMS values) can be defined as

$$
\bar{u}_{i, r m s}^{+} \stackrel{\text { def }}{=}\left\langle\left(\frac{\bar{u}_{i}-\left\langle\bar{u}_{i}\right\rangle}{u_{\tau}^{a}}\right)^{2}\right\rangle^{1 / 2}=\frac{\left\langle\bar{u}_{i}^{\prime \prime 2}\right\rangle^{1 / 2}}{u_{\tau}^{a}}
$$

for $i=1,2$ and 3 , and

$$
\bar{\theta}_{r m s}^{+} \stackrel{\text { def }}{=}\left\langle\left(\frac{\bar{\theta}-\langle\bar{\theta}\rangle}{\theta_{\tau}^{a}}\right)^{2}\right\rangle^{1 / 2}=\frac{\left\langle\bar{\theta}^{\prime \prime 2}\right\rangle^{1 / 2}}{\theta_{\tau}^{a}},
$$

respectively, where $u_{\tau}^{a}=\left(u_{\tau h}+u_{\tau c}\right) / 2$ and $\theta_{\tau}^{a}=\left(\theta_{\tau h}+\theta_{\tau c}\right) / 2$ are the averaged wall friction velocity and temperature over both the hot and cold walls.

Figure 3.11 compares the predicted resolved velocity fluctuations in the stream- 


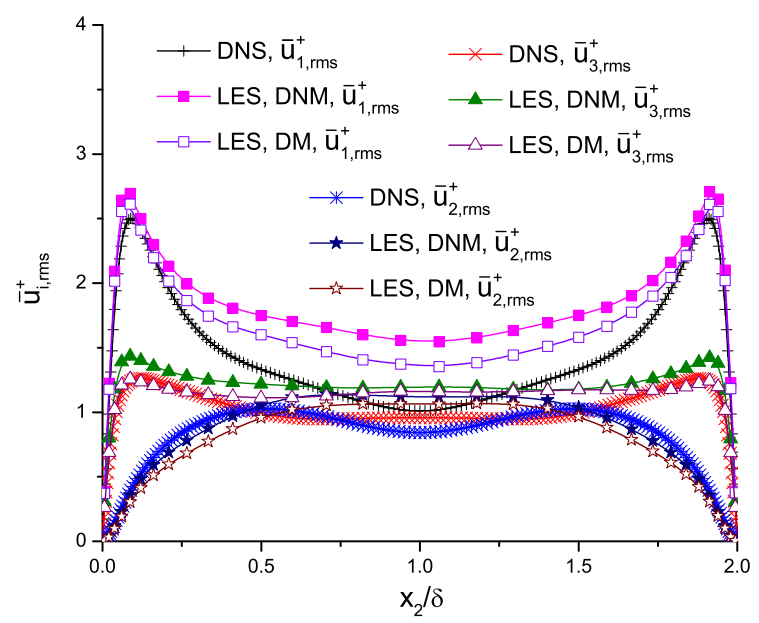

(a) $R e_{\tau}=150, R o_{\tau}=15$

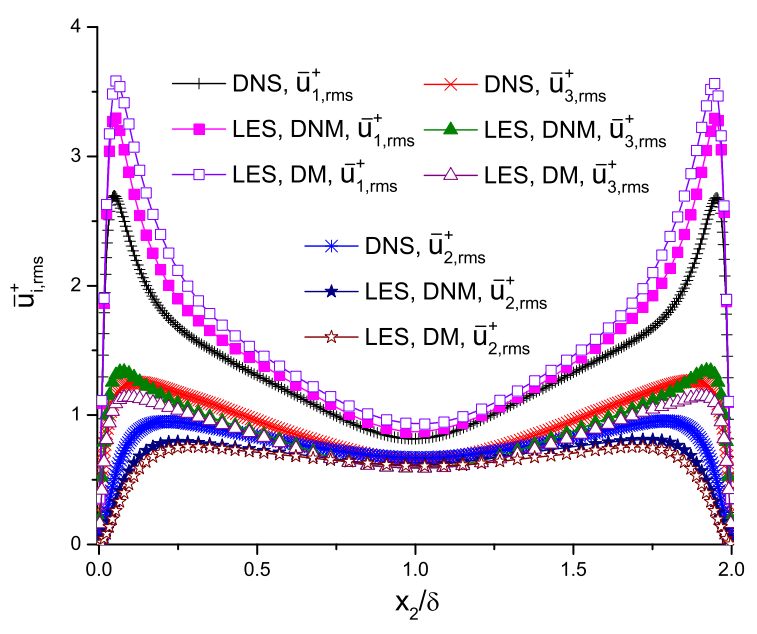

(b) $R e_{\tau}=300, R o_{\tau}=2.5$

FIGURE 3.11: Resolved velocity fluctuations (predicted in conjunction with the DFLTDM).

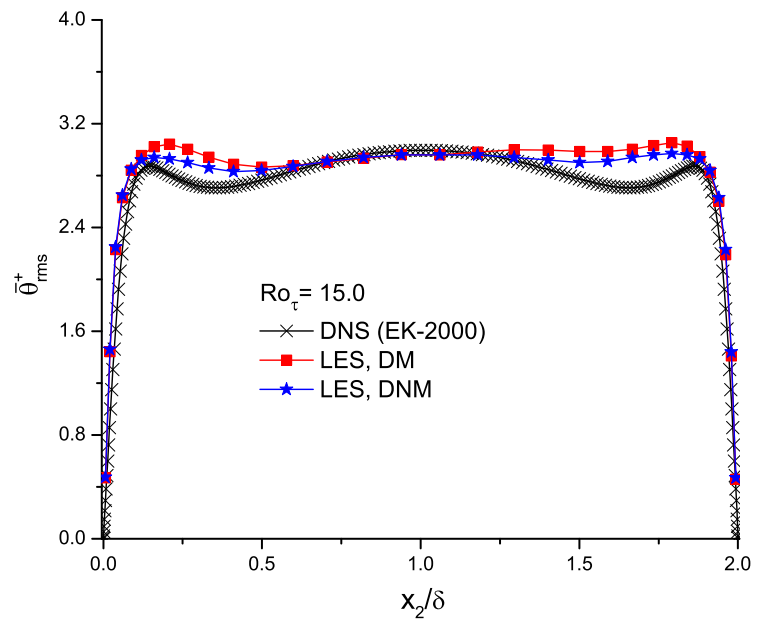

(a) $R e_{\tau}=150$

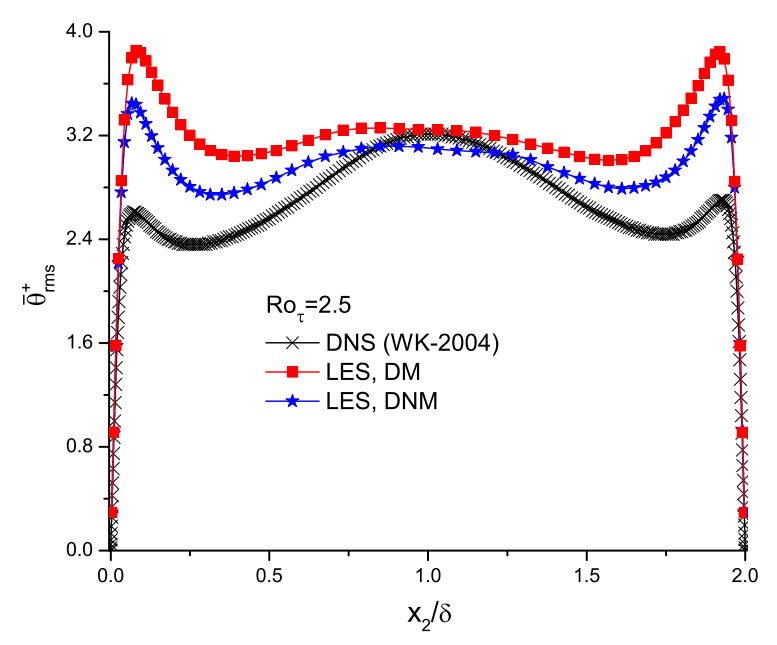

(b) $R e_{\tau}=300$

FIGURE 3.12: Resolved temperature fluctuations for $R o_{\tau}=15.0$ at $R e_{\tau}=150$, $R o_{\tau}=2.5$ at $R e_{\tau}=300$ (predicted in conjunction with the DFLTDM).

wise, wall-normal and spanwise direction with the available DNS data for two Reynolds numbers. The predicted temperature fluctuation is displayed in Fig. 3.12. As shown in Fig. 3.11, the basic trends of each RMS profile at $R e_{\tau}=150$ and 300 are captured by LES approach in comparison with the DNS data. However, differences are observed. For instance, at $R e_{\tau}=150$, the LES approach tends to overpredict the streamwise and spanwise RMS velocities $\left(\bar{u}_{1, r m s}^{+}\right.$and $\left.\bar{u}_{3, r m s}^{+}\right)$in the central plane of the channel and wall-normal RMS $\left(\bar{u}_{2, r m s}^{+}\right)$in the near-wall region. At $R e_{\tau}=300$, 
it tends to slightly underpredict the wall-normal RMS velocity $\left(\bar{u}_{2, r m s}^{+}\right)$in the entire flow region and overpredict the streamwise RMS velocity $\left(\bar{u}_{1, r m s}^{+}\right)$in the near-wall region. As indicated by Winckelmans et al. [69], these differences are expected. This is because in the conventional implicit-filtering LES approach used in the research, numerical simulations are conducted based on a trace-free SGS stress model. Owing to the isotropic property of the three normal components of the SGS stress tensor, they are absorbed into the pressure term. Hence, information on the trace of the SGS stress tensor in the conventional implicit LES approach is not explicitly provided unless an additional SGS $k$-equation is solved (which however demands further SGS modelling). In Fig. 3.12(a), in comparison with the DNS data at $R o_{\tau}=15$ and $R e_{\tau}=150$, the LES approach only slightly overpredicts the temperature fluctuation in the near-wall region. For $R o_{\tau}=2.5$ and $R e_{\tau}=300$, as shown in Fig. 3.12(b), LES overpredicts the temperature fluctuation in the near-wall region and slightly underpredictes it in the core flow region in comparison with the DNS data.

In order to compare the influence of different SGS stress models (i.e., the DM and DNM) on the prediction of the scalar field, all numerical simulations in Figs. 3.11 and 3.12 were performed based on the same SGS HF model DFLTDM. As discussed before, for the prediction of the first momentum of the resolved velocity and temperature, the DNM and DM gave similar satisfactory results at two different Reynolds numbers. While for the prediction of the second momentum statistics, slight differences exist between different SGS stress models, especially in terms of the prediction of the streamwise RMS velocity $\bar{u}_{1, r m s}$ and the temperature fluctuation $\bar{\theta}_{r m s}$. The statistics obtained using the DNM are in better conformance with the DNS data than those obtained using the conventional DM.

Figure 3.13 shows the profile of the resolved temperature intensity at various rotation numbers. It is observed that at the non-rotating case, three peaks appear across the channel. After the rotation is imposed upon the system, the peak near the central plane reduces dramatically in comparison with the other two peaks near the wall. By comparing Figs. 3.13 (a) and (b), it is found that in response to the system 


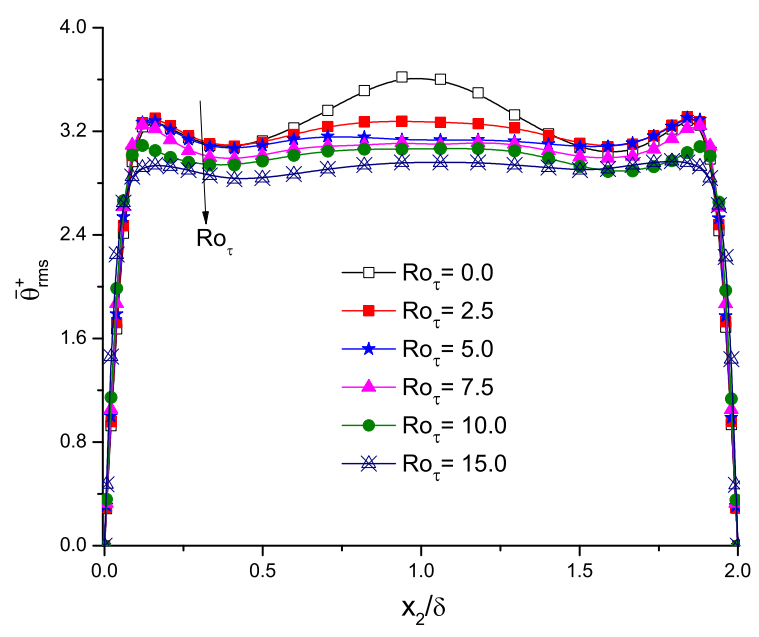

(a) $R e_{\tau}=150$

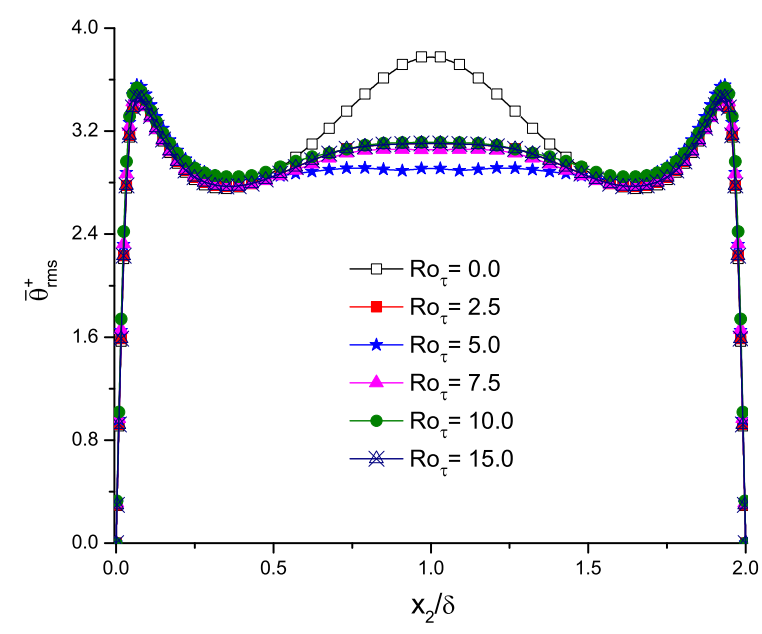

(b) $R e_{\tau}=300$

FIGURE 3.13: Resolved temperature fluctuations at different rotation numbers (predicted using the DNM and DFLTDM).

rotation, the change of RMS temperature profile is more obvious at a lower Reynolds number.

\subsection{Visualization of the Flow Field}

In this section, the contours of the wall-normal velocity field and the streamwise vorticity field are visualized in order to further understand the structures of the heated rotating channel flows. To demonstrate, the thermal flow field for $R e_{\tau}=300$ and $R o_{\tau}=15$ is used. In Fig. 3.14, the instantaneous iso-surface of the wall-normal velocity field for $R e_{\tau}=300$ and $R o_{\tau}=15$ is visualized. Streamwise elongated vortical flow structures are prevalent in the channel. In the near-wall region, existence of streaks is evident. Figure 3.15 shows the instantaneous streamwise vorticity field in the $\left(x_{1}\right.$, $x_{3}$ )-plane in the near-wall region (at $x_{2} / \delta=1.8$ ). From the figure, it is observed that driven by the mean streamwise pressure gradient and Coriolis forces, the flow shows fine turbulent structures even in the near-wall region. Local vortical structures rotating in both clockwise and counter-clockwise (with respect to the streamwise direction) coexist in the plane. 


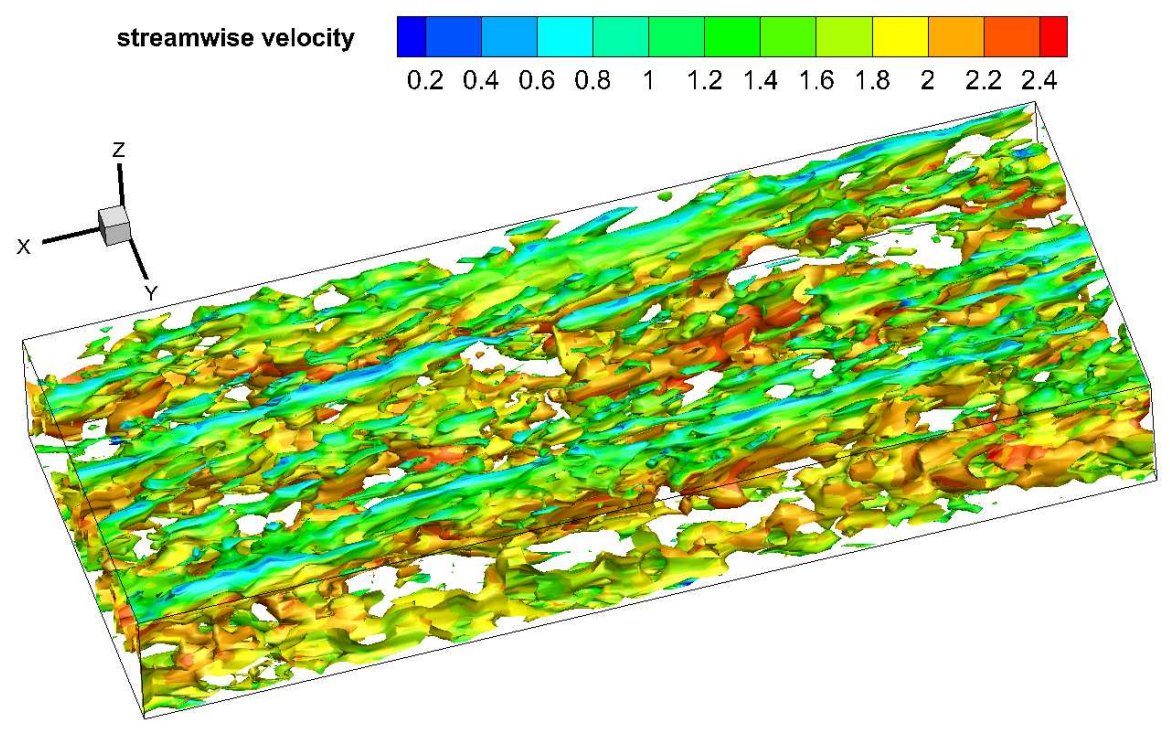

FIGURE 3.14: Iso-surface of the wall-normal velocity field for $R e_{\tau}=300$ and $R o_{\tau}=$ 15.

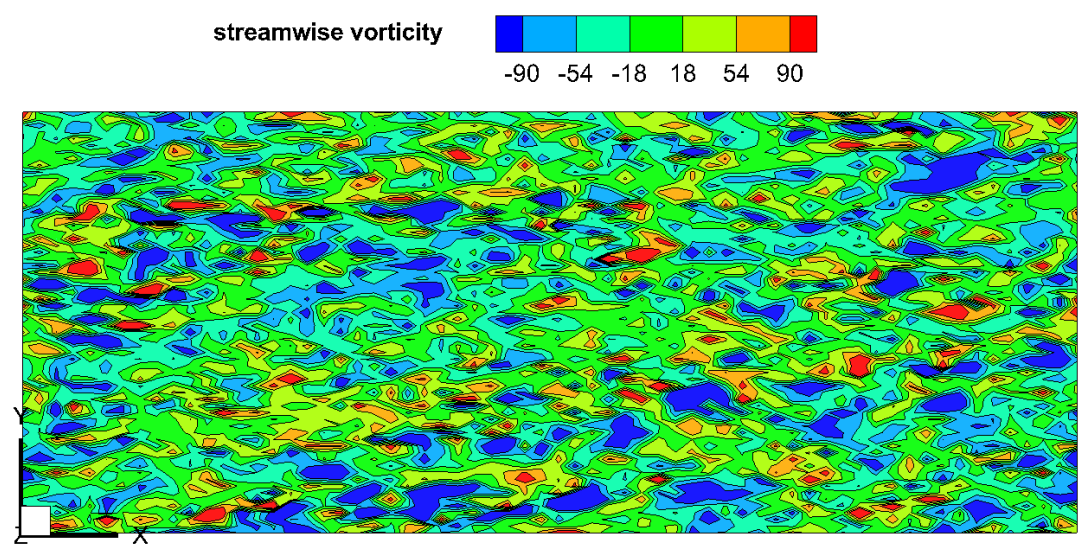

FIGURE 3.15: Contour of the streamwise vorticity field in the $\left(x_{1}, x_{3}\right)$-plane $\left(x_{2} / \delta=\right.$ 1.8 , near the wall) for $R e_{\tau}=300$ and $R o_{\tau}=15$. 


\section{Chapter 4}

\section{Rotation Effects on Transport of Turbulent Quantities}

In the previous chapter, fundamental features of the resolved velocity and temperature fields have been systematically analyzed in terms of their mean and RMS values. In this chapter, some advanced physical features will be thoroughly investigated to include the transport of turbulent stresses, TKE and heat fluxes, and forward and backward scatter of local KE fluxes between the resolved and subgrid scales.

\subsection{Transport of Resolved Turbulent Stresses and TKE}

\subsubsection{Rotation Effect on the Resolved Turbulent Stresses}

In the previous analysis, it has been demonstrated that the level of the first- and second-order statistics of the resolved velocity and temperature fields are drastically changed in the flow under the streamwise system rotation. According to Xun et al. [14], the effects of the Coriolis forces on the resolved turbulent stresses and TKE can be further studied using their transport equations. As revealed in the experimental study of Johnston et al. [23] and DNS study of Kristoffersen and Andersson [35], the production terms in the transport equations of the resolved turbulent stresses 
TABLE 4.1: Production terms due to the mean turbulent shear $\left(P_{i j}\right)$ and rotation $\left(G_{i j}\right)$ stresses for a fullydeveloped rotating plane channel flow.

\begin{tabular}{c|cc}
\hline$i j$ & $P_{i j}$ & $G_{i j}$ \\
\hline 11 & $-2\left\langle\bar{u}_{1}^{\prime \prime} \bar{u}_{2}^{\prime \prime}\right\rangle\left(d\left\langle\bar{u}_{1}\right\rangle / d x_{2}\right)$ & 0 \\
22 & 0 & $4 \Omega\left\langle\bar{u}_{2}^{\prime \prime} \bar{u}_{3}^{\prime \prime}\right\rangle$ \\
33 & $-2\left\langle\bar{u}_{2}^{\prime \prime} \bar{u}_{3}^{\prime \prime}\right\rangle\left(d\left\langle\bar{u}_{3}\right\rangle / d x_{2}\right)$ & $-4 \Omega\left\langle\bar{u}_{2}^{\prime \prime} \bar{u}_{3}^{\prime \prime}\right\rangle$ \\
12 & $-\left\langle\bar{u}_{2}^{\prime \prime 2}\right\rangle\left(d\left\langle\bar{u}_{1}\right\rangle / d x_{2}\right)$ & $2 \Omega\left\langle\bar{u}_{1}^{\prime \prime} \bar{u}_{3}^{\prime \prime}\right\rangle$ \\
13 & $-\left\langle\bar{u}_{1}^{\prime \prime} \bar{u}_{2}^{\prime \prime}\right\rangle\left(d\left\langle\bar{u}_{3}\right\rangle / d x_{2}\right)-\left\langle\bar{u}_{2}^{\prime \prime} \bar{u}_{3}^{\prime \prime}\right\rangle\left(d\left\langle\bar{u}_{1}\right\rangle / d x_{2}\right)$ & $-2 \Omega\left\langle\bar{u}_{1}^{\prime \prime} \bar{u}_{2}^{\prime \prime}\right\rangle$ \\
23 & $-\left\langle\bar{u}_{2}^{\prime \prime 2}\right\rangle\left(d\left\langle\bar{u}_{3}\right\rangle / d x_{2}\right)$ & $2 \Omega\left(\left\langle\bar{u}_{3}^{\prime \prime 2}\right\rangle-\left\langle\bar{u}_{2}^{\prime \prime 2}\right\rangle\right)$ \\
\hline
\end{tabular}

have a significant influence on the absolute value and transport of the resolved turbulent shear stresses (i.e., $\left.\left\langle\bar{u}_{i}^{\prime \prime} \bar{u}_{j}^{\prime \prime}\right\rangle\right)$ and TKE (i.e., $\left.1 / 2\left\langle\bar{u}_{i}^{\prime \prime} \bar{u}_{i}^{\prime \prime}\right\rangle\right)$. As shown in Appendix A, there are two production terms related to the resolved turbulent shear and rotation stresses in the transport equations for the resolved turbulent stresses. The production term due to the resolved turbulent shear stresses is

$$
P_{i j}=-\left\langle\bar{u}_{i}^{\prime \prime} \bar{u}_{2}^{\prime \prime}\right\rangle \frac{\partial\left\langle\bar{u}_{j}\right\rangle}{\partial x_{2}}-\left\langle\bar{u}_{j}^{\prime \prime} \bar{u}_{2}^{\prime \prime}\right\rangle \frac{\partial\left\langle\bar{u}_{i}\right\rangle}{\partial x_{2}}
$$

and the production term due to the rotation stresses is determined as

$$
G_{i j}=2 \Omega\left(\varepsilon_{i k 1}\left\langle\bar{u}_{k}^{\prime \prime} \bar{u}_{j}^{\prime \prime}\right\rangle+\varepsilon_{j k 1}\left\langle\bar{u}_{k}^{\prime \prime} \bar{u}_{i}^{\prime \prime}\right\rangle\right)
$$

From Eq. (4.1), it is understood that the value of $P_{i j}$ is directly influenced by the resolved turbulent shear stresses and indirectly by the system rotation if the local wall-normal gradient of the mean velocity is nonzero. To facilitate the interpretation of the results in Figs. 4.1 - 4.6, Eqs. (4.1) and (4.2) are written in component forms in Table 4.1.

As shown in Fig. 4.1, in the central core of the channel, the value of the resolved streamwise turbulence intensity $\bar{u}_{1, r m s}^{+}$increases as the rotation number $R o_{\tau}$ increases. 


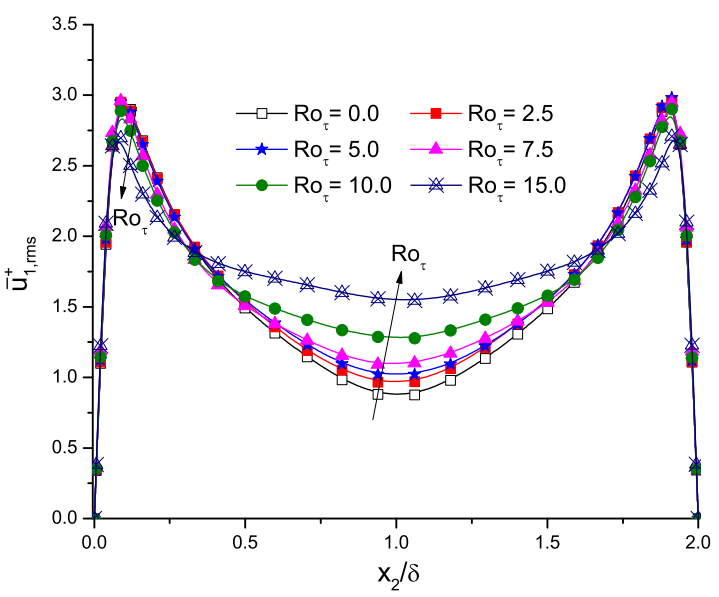

(a) $R e_{\tau}=150$

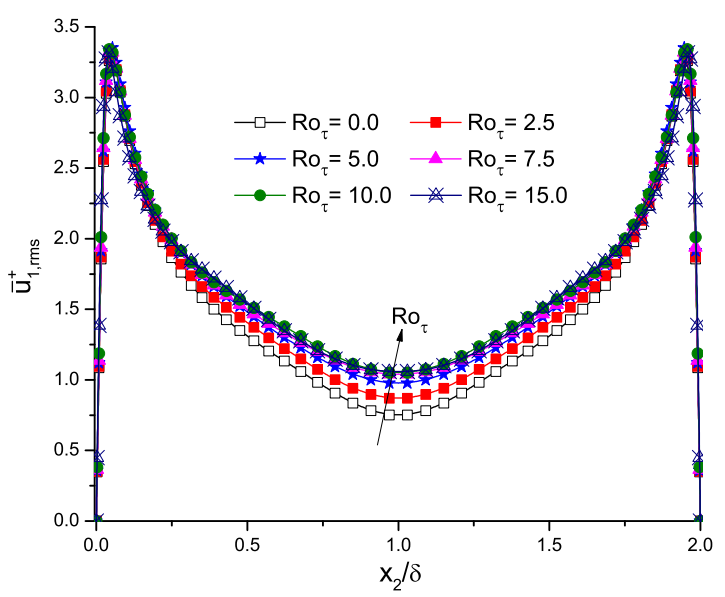

(b) $R e_{\tau}=300$

FIGURE 4.1: Resolved streamwise velocity fluctuations at various rotation numbers (predicted using the DNM and DFLTDM).

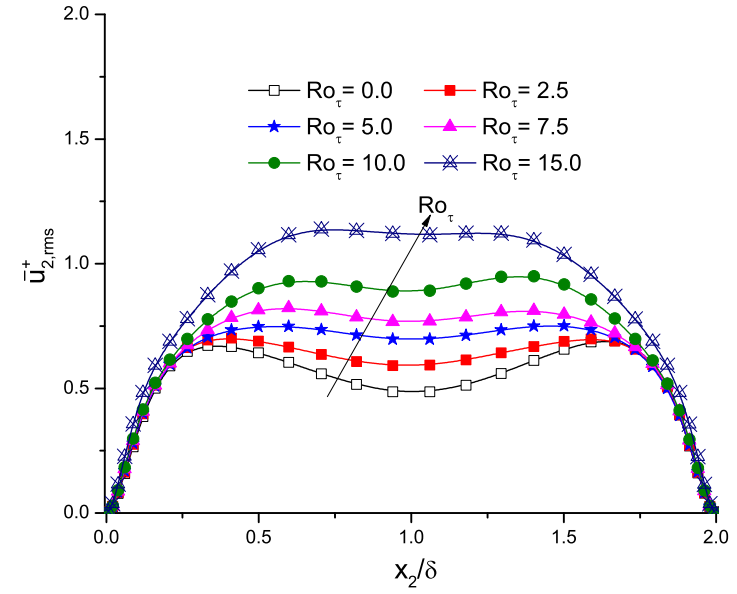

(a) $R e_{\tau}=150$

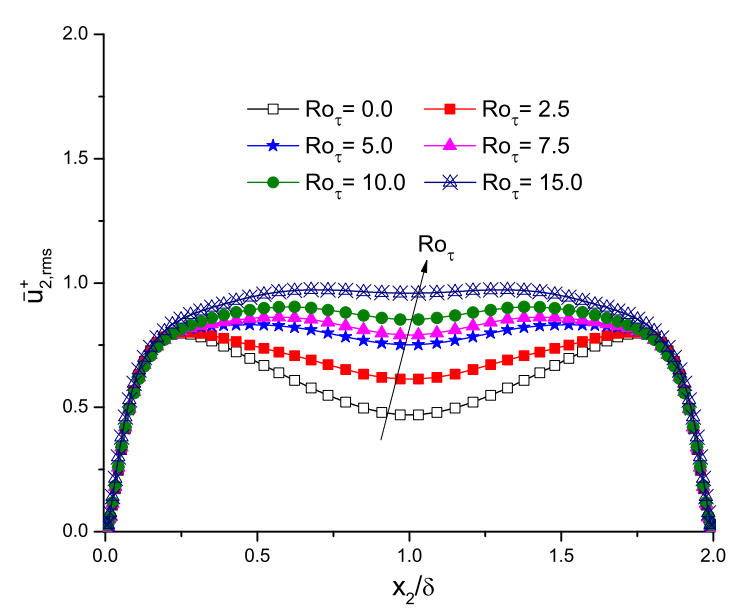

(b) $R e_{\tau}=300$

FIGURE 4.2: Resolved wall-normal velocity fluctuations at various rotation numbers (predicted using the DNM and DFLTDM).

However, the profiles of $\bar{u}_{1, r m s}^{+}$peak and collapse in the near-wall region. The physical mechanism underlying this near-wall behaviour can be explained as follows. From Table 4.1, the production term for $\left\langle\bar{u}_{1}^{\prime \prime} \bar{u}_{1}^{\prime \prime}\right\rangle$ is determined as $P_{11}=-2\left\langle\bar{u}_{1}^{\prime \prime} \bar{u}_{2}^{\prime \prime}\right\rangle\left(d\left\langle\bar{u}_{1}\right\rangle / d x_{2}\right)$ $\left(G_{11} \equiv 0\right)$. From Figs. 3.4 and 4.4, it is understood that the sign of $d\left\langle\bar{u}_{1}\right\rangle / d x_{2}$ and that of $\left\langle\bar{u}_{1}^{\prime \prime} \bar{u}_{2}^{\prime \prime}\right\rangle$ are strictly opposite to each other across the channel, due to the fact that the profiles of $\left\langle\bar{u}_{1}\right\rangle$ and $\left\langle\bar{u}_{1}^{\prime \prime} \bar{u}_{2}^{\prime \prime}\right\rangle$ are symmetrical and skew-symmetrical about $x_{2} / \delta=1$ in the wall-normal direction, respectively. Furthermore, the magnitudes of 


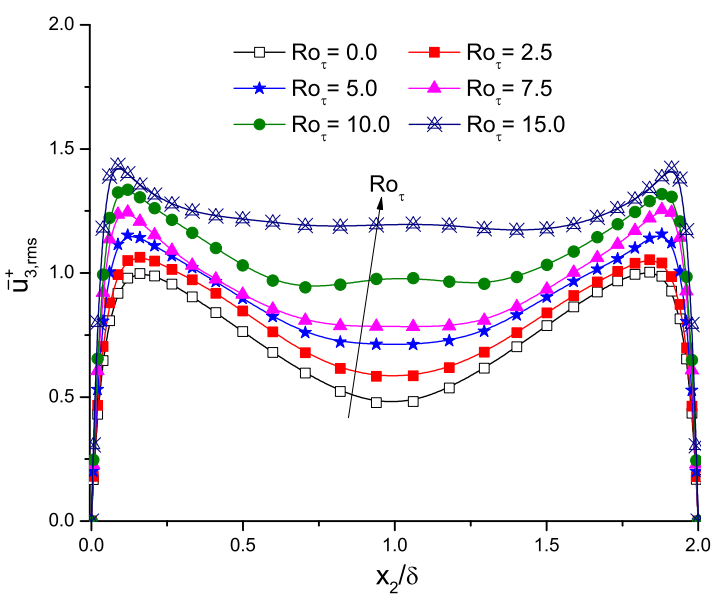

(a) $R e_{\tau}=150$

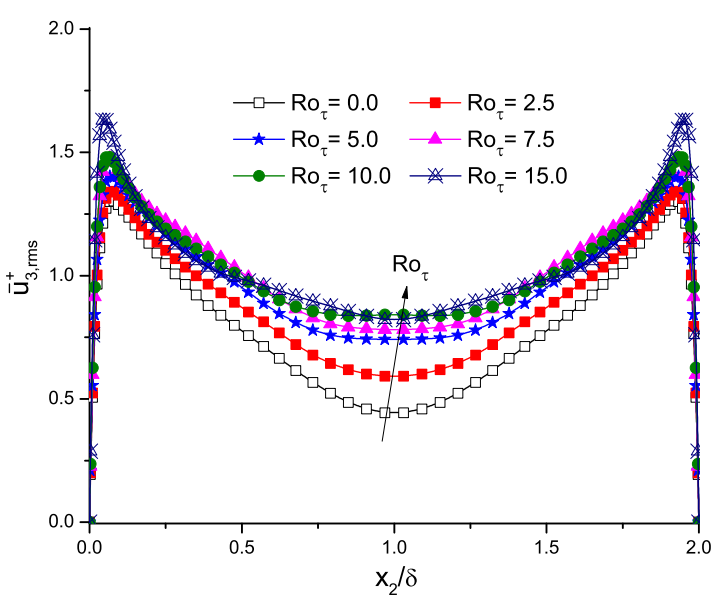

(b) $R e_{\tau}=300$

FIGURE 4.3: Resolved spanwise velocity fluctuations at various rotation numbers (predicted using the DNM and DFLTDM).

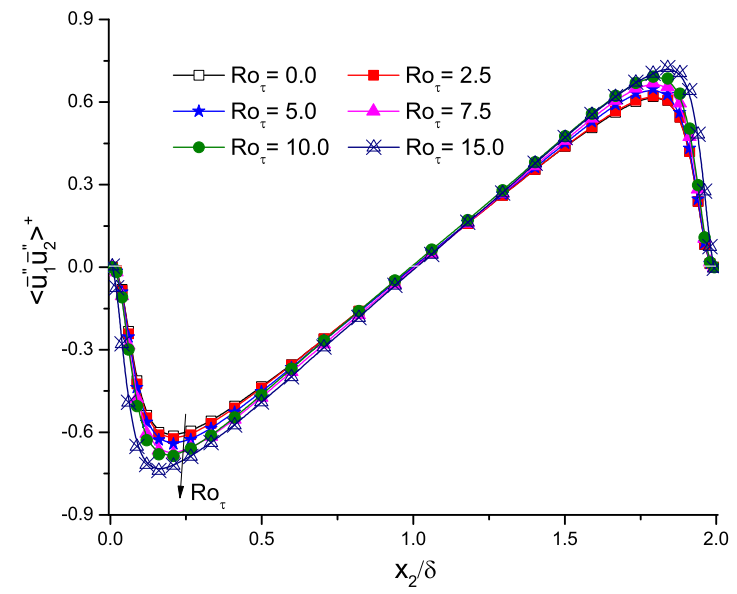

(a) $R e_{\tau}=150$

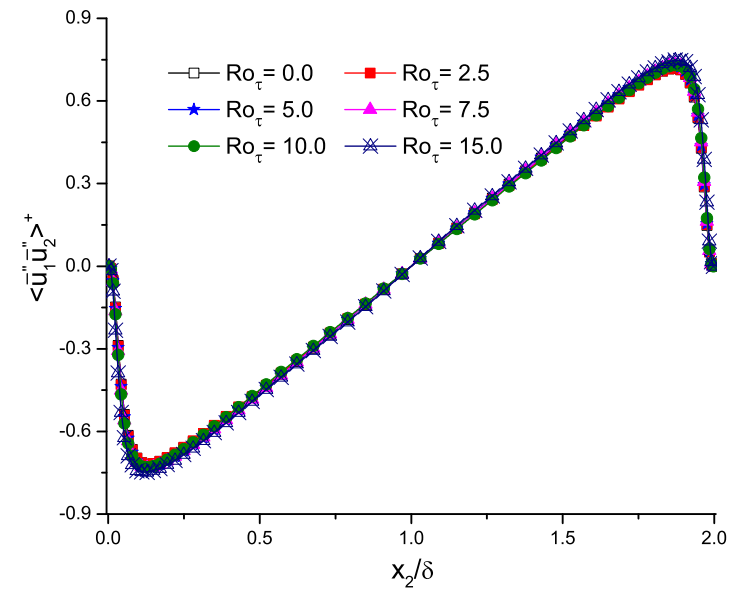

(b) $R e_{\tau}=300$

FIGURE 4.4: Resolved Reynolds shear stress $\left\langle\bar{u}_{1}^{\prime \prime} \bar{u}_{2}^{\prime \prime}\right\rangle^{+}$at various rotation numbers (predicted using the DNM and DFLTDM).

both $\left\langle\bar{u}_{1}^{\prime \prime} \bar{u}_{2}^{\prime \prime}\right\rangle$ and $d\left\langle\bar{u}_{1}\right\rangle / d x_{2}$ peaks in the near wall region. For these reasons, $P_{11}>0$ holds across the channel and $\bar{u}_{1, r m s}^{+}$necessarily peaks in the near-wall region.

In the central region of the channel, the direct effects of system rotation are expected to increase $\left\langle\bar{u}_{2}^{\prime \prime 2}\right\rangle$ (or $\bar{u}_{2, r m s}^{+}$) because the total production rate is positive, i.e. $P_{22}+G_{22}=G_{22}>0$. From Table 4.1, it is understood that $G_{22}=4 \Omega\left\langle\bar{u}_{2}^{\prime \prime} \bar{u}_{3}^{\prime \prime}\right\rangle$ and $\left\langle\bar{u}_{2}^{\prime \prime} \bar{u}_{3}^{\prime \prime}\right\rangle>0$ holds across channel in the wall-normal direction (see Fig. 4.5). As shown in Fig. 4.2, the value of $\bar{u}_{2, r m s}^{+}$indeed increases monotonically with the rotation 


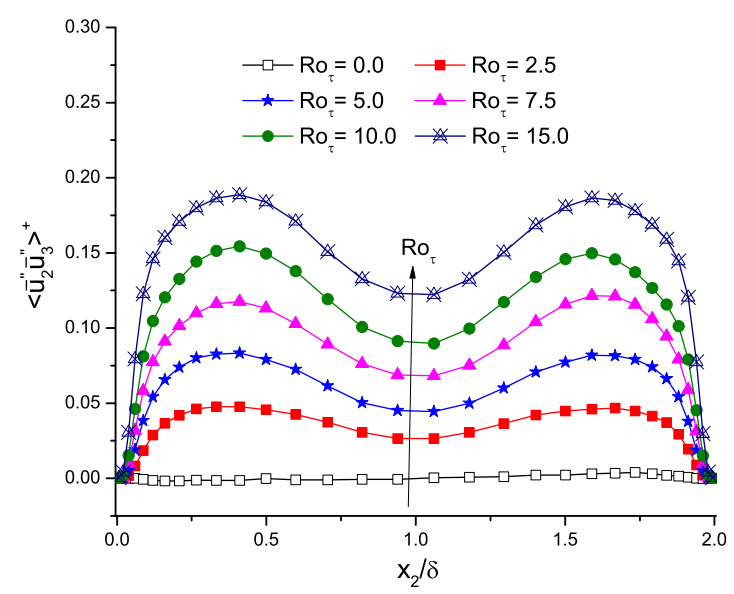

(a) $R e_{\tau}=150$

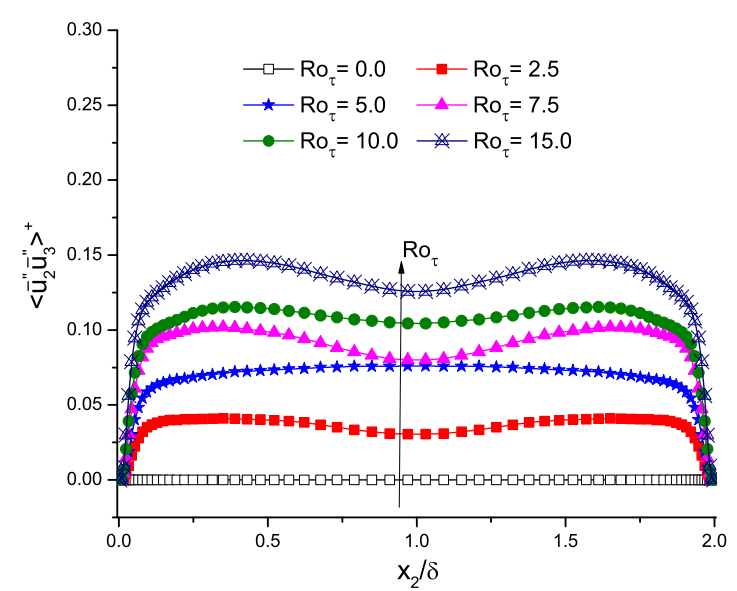

(b) $R e_{\tau}=300$

FIGURE 4.5: Resolved Reynolds shear stress $\left\langle\bar{u}_{2}^{\prime \prime} \bar{u}_{3}^{\prime \prime}\right\rangle^{+}$at various rotation numbers (predicted using the DNM and DFLTDM).

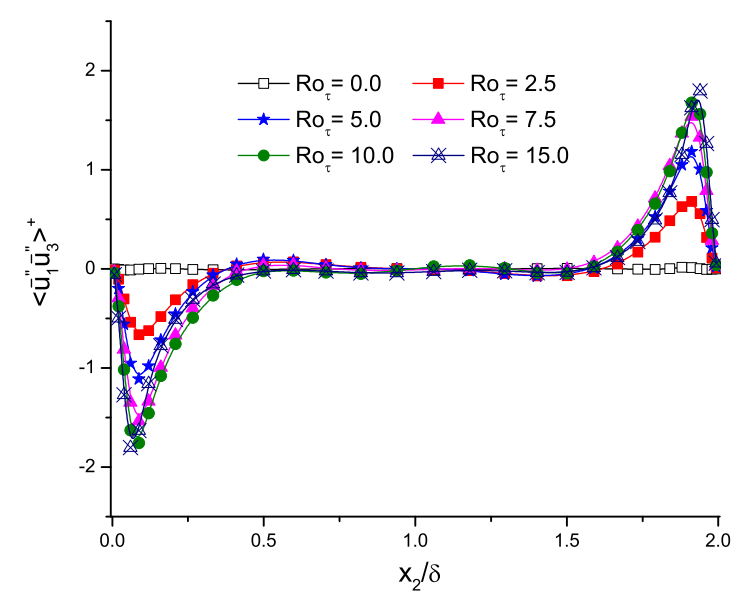

(a) $R e_{\tau}=150$

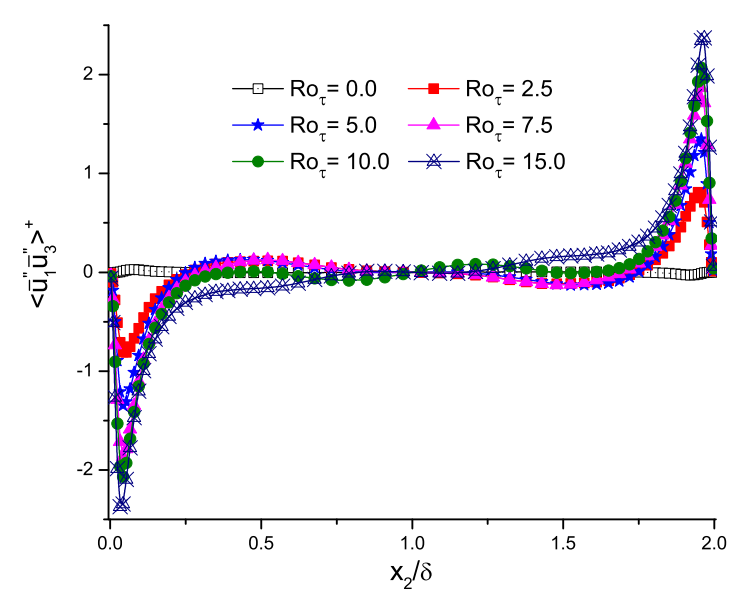

(b) $R e_{\tau}=300$

FIGURE 4.6: Resolved Reynolds shear stress $\left\langle\bar{u}_{1}^{\prime \prime} \bar{u}_{3}^{\prime \prime}\right\rangle^{+}$at various rotation numbers (predicted using the DNM and DFLTDM).

number in the central core of the channel in response to the total production rate: $P_{22}+G_{22}=G_{22}$.

Figure 4.3 shows that the value of $\bar{u}_{3, r m s}^{+}$increases with the rotation number, especially in the central core of the channel. The physical mechanism underlying this pattern is complicated, because it relates to the four layers of opposite stratifications of the secondary flow in the cross-stream plane (indicated by the involvement of $\left\langle\bar{u}_{3}\right\rangle$ in the formula for $P_{33}$ in Table 4.1). 
Near the top wall $\left(x_{2} / \delta=2\right), P_{12}>0$ (because $d\left\langle\bar{u}_{1}\right\rangle / d x_{2}<0$, see Fig. 3.4) and $G_{12}>0$ (because $\left\langle\bar{u}_{1}^{\prime \prime} \bar{u}_{3}^{\prime \prime}\right\rangle>0$ and $\Omega>0$ ). Furthermore, from Figs. 3.4 and 4.2, it is understood that the magnitudes of $d\left\langle\bar{u}_{1}\right\rangle / d x_{2}$ and $\left\langle\bar{u}_{2}^{\prime \prime 2}\right\rangle$ are the largest in the near-wall region, which necessarily result in a peak value in $P_{12}$ in the region near the top wall. From Fig. 4.6, it is observed that the magnitude of $\left\langle\bar{u}_{1}^{\prime \prime} \bar{u}_{3}^{\prime \prime}\right\rangle$ peaks in the near-wall region and this will result in a peak in $G_{12}$ (because $G_{12} \propto\left\langle\bar{u}_{1}^{\prime \prime} \bar{u}_{3}^{\prime \prime}\right\rangle$ and $\Omega$ ). As a consequence, in the region near the top wall, the value of $P_{12}+G_{12}$ is positive and enhanced by the positive peak values of $P_{12}$ and $G_{12}$, which then further results in a positive peak value in the magnitude of the turbulent shear stress component $\left\langle\bar{u}_{1}^{\prime \prime} \bar{u}_{2}^{\prime \prime}\right\rangle$. Because $\left(P_{12}+G_{12}\right) \propto G_{12} \propto \Omega$, it is also anticipated that the magnitude of $\left\langle\bar{u}_{1}^{\prime \prime} \bar{u}_{2}^{\prime \prime}\right\rangle$ increases with the rotation number $R o_{\tau}$. The above theoretical analysis of the behavior of $\left\langle\bar{u}_{1}^{\prime \prime} \bar{u}_{2}^{\prime \prime}\right\rangle$ in the region near the top wall agrees well with the numerical predictions shown in Fig. 4.4. Because the profile of $\left\langle\bar{u}_{1}^{\prime \prime} \bar{u}_{2}^{\prime \prime}\right\rangle$ is symmetrical about the central wall-normal plane $x_{2} / \delta=1$, similar analysis can be conducted to understand its behavior in the region near the bottom wall $\left(x_{2} / \delta=0\right)$.

All these trends can be observed at two Reynolds numbers. By comparing the figure for $R e_{\tau}=150$ with that for $R e_{\tau}=300$, it is found that the variation tendencies are more strongly expressed at the lower Reynolds number flow while the flow at the higher Reynolds number is less sensitive to the imposed system rotation. This is because at a given rotation number, the higher the Reynolds number, the larger the turbulent shear stresses (in comparison with the rotation stresses and Coriolis forces). In other words, at a given rotation number, the flow pattern becomes more towards Poiseuille type as the Reynolds number increases.

\subsubsection{Rotation Effect on the Resolved TKE}

The resolved TKE is defined as

$$
\left\langle q^{2}\right\rangle=\frac{1}{2}\left(\left\langle\bar{u}_{1}^{\prime \prime 2}\right\rangle+\left\langle\bar{u}_{2}^{\prime \prime 2}\right\rangle+\left\langle\bar{u}_{3}^{\prime \prime 2}\right\rangle\right) \quad .
$$


The production term for the resolved TKE can be obtained by taking one half of the summation resultant of the corresponding elements in the first three rows of Table 4.1, i.e.

$$
\left\langle P_{q^{2}}\right\rangle+\left\langle G_{q^{2}}\right\rangle=-\left\langle\bar{u}_{1}^{\prime \prime} \bar{u}_{2}^{\prime \prime}\right\rangle \frac{d\left\langle\bar{u}_{1}\right\rangle}{d x_{2}}-\left\langle\bar{u}_{2}^{\prime \prime} \bar{u}_{3}^{\prime \prime}\right\rangle \frac{d\left\langle\bar{u}_{3}\right\rangle}{d x_{2}}+0
$$

where

$$
\left\langle P_{q^{2}}\right\rangle=\frac{1}{2}\left(\left\langle P_{11}\right\rangle+\left\langle P_{22}\right\rangle+\left\langle P_{33}\right\rangle\right)
$$

and

$$
\left\langle G_{q^{2}}\right\rangle=\frac{1}{2}\left(\left\langle G_{11}\right\rangle+\left\langle G_{22}\right\rangle+\left\langle G_{33}\right\rangle\right)
$$

Equation (4.4) clearly shows that the production of the resolved TKE $\left\langle q^{2}\right\rangle$ is not explicitly dependent upon $\Omega$ but rather the resolved turbulent shear stresses $\left(\left\langle\bar{u}_{1}^{\prime \prime} \bar{u}_{2}^{\prime \prime}\right\rangle\right.$ and $\left.\left\langle\bar{u}_{2}^{\prime \prime} \bar{u}_{3}^{\prime \prime}\right\rangle\right)$ and mean viscous shear stresses (indicated by $d\left\langle\bar{u}_{1}\right\rangle / d x_{2}$ and $d\left\langle\bar{u}_{3}\right\rangle / d x_{2}$, based on Newton's law for shear flows). However, as shown in Table 4.1 and Figs. 3.4 and 3.5, all these resolved quantities are influenced by the system rotation $\Omega$. Because $\left\langle P_{22} \equiv 0\right\rangle,\left\langle P_{q^{2}}\right\rangle$ is one half of $\left(\left\langle P_{11}\right\rangle+\left\langle P_{33}\right\rangle\right)$, which is the sum of the production terms for $\left\langle\bar{u}_{1}^{\prime \prime 2}\right\rangle$ and $\left\langle\bar{u}_{3}^{\prime \prime 2}\right\rangle$. Therefore, it is anticipated that the resolved TKE $\left\langle q^{2}\right\rangle$ will also exhibits a behavior that is similar to that of the combination of the resolved streamwise and spanwise turbulent normal stresses $\left\langle\bar{u}_{1}^{\prime \prime 2}\right\rangle$ and $\left\langle\bar{u}_{3}^{\prime \prime 2}\right\rangle$. Furthermore, because the magnitude of $\left\langle\bar{u}_{1}^{\prime \prime 2}\right\rangle$ is much larger than that of $\left\langle\bar{u}_{3}^{\prime \prime 2}\right\rangle$ (which is evident by comparing Figs. 4.1 and 4.3), it is inferred that the profile of $\operatorname{TKE}\left\langle q^{2}\right\rangle$ is dominated by $\left\langle\bar{u}_{1}^{\prime \prime 2}\right\rangle$, and so, it tends to exhibit a similar pattern to that of $\left\langle\bar{u}_{1}^{\prime \prime 2}\right\rangle$. The profiles of TKE at various rotation numbers obtained from numerical simulations are displayed in Fig. 4.7, which verifies the above analysis. Figure 4.7 clearly shows that the value of $\left\langle q^{2}\right\rangle$ peaks in the near-wall region, and increases monotonically as $R o_{\tau}$ increases in the central core of the domain. Indeed, there exists a great similarity between the profile of $\left\langle q^{2}\right\rangle$ shown in Fig. 4.7 and that of $\left\langle\bar{u}_{1}^{\prime \prime 2}\right\rangle^{1 / 2}$ shown in Fig. 4.1. By comparing Figs. 4.7(a) and (b), it is observed that the peak maginitude of the TKE in the nearwall region is much larger at $R e_{\tau}=300$ which in consistent with the fact that flow is more turbulent at the higher Reynolds number. 


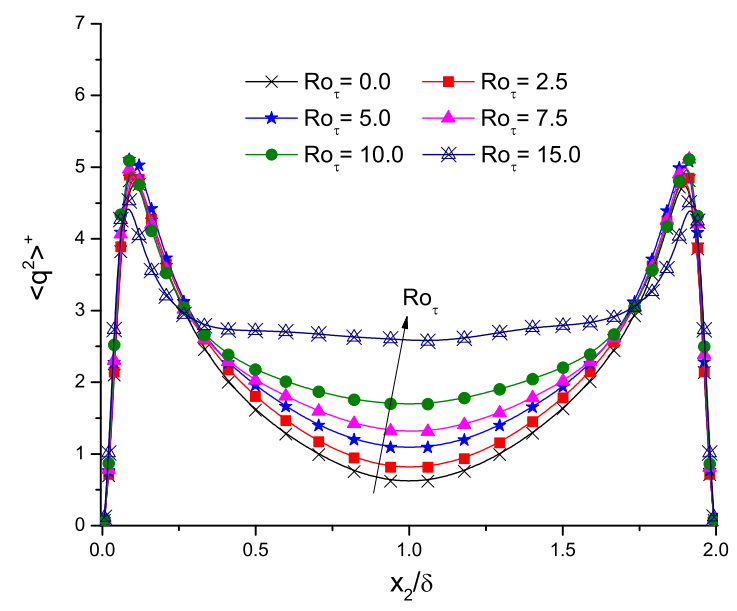

(a) $R e_{\tau}=150$

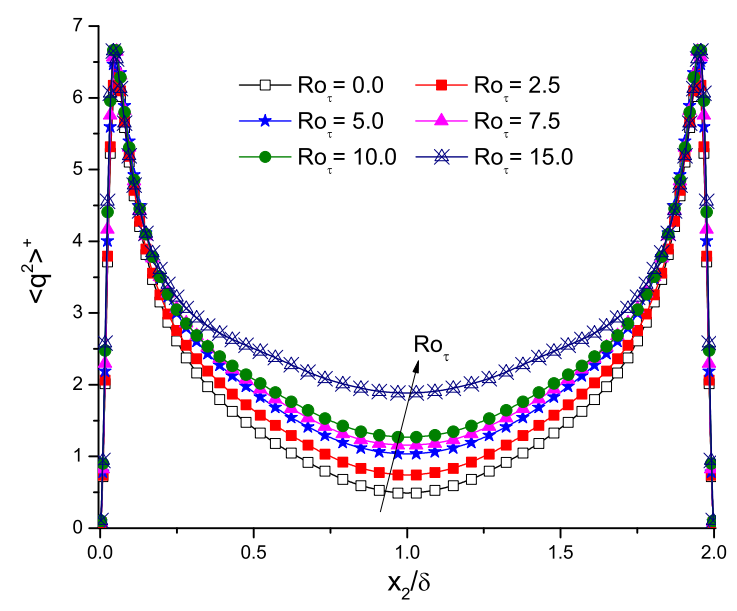

(b) $R e_{\tau}=300$

FIGURE 4.7: Resolved turbulent kinetic energy at various rotation numbers (nondimensionalized by $\tau_{w}^{a} / \rho$, predicted using the DNM and DFLTDM).

\subsection{Budgets of Shear Stresses and Heat Fluxes}

In order to study the predictive performance of the LES approach in terms of the momentum balance and energy balance, the budget of the shear stresses and that of the heat fluxes across the channel need to be examined.

\subsubsection{Budget of Shear Stresses}

On assuming that the flow is statistically stationary and homogeneous in the $\left(x_{1}, x_{3}\right)$ plane, the equation that expresses the balance between the time- and plane-averaged viscous, turbulent and SGS shear stresses at an arbitrary wall-normal location $x_{2}$ can be derived from the filtered streamwise momentum equation, viz.

$$
\nu \frac{\partial\left\langle\bar{u}_{1}\right\rangle}{\partial x_{2}}-\left\langle\bar{u}_{1}^{\prime \prime} \bar{u}_{2}^{\prime \prime}\right\rangle-\left\langle\tau_{12}\right\rangle=\frac{1}{\rho} \frac{\partial\langle\bar{p}\rangle}{\partial x_{1}} x_{2}+\frac{\tau_{w h}}{\rho}
$$

The three terms on the LHS of Eq. (4.7) represent the resolved viscous shear stress, resolved turbulent shear stress, and SGS shear stress, respectively. The two terms on the RHS of the equation correspond to the resolved integrated shear force due to the mean pressure gradient, and the resolved viscous shear stress at the hot wall 


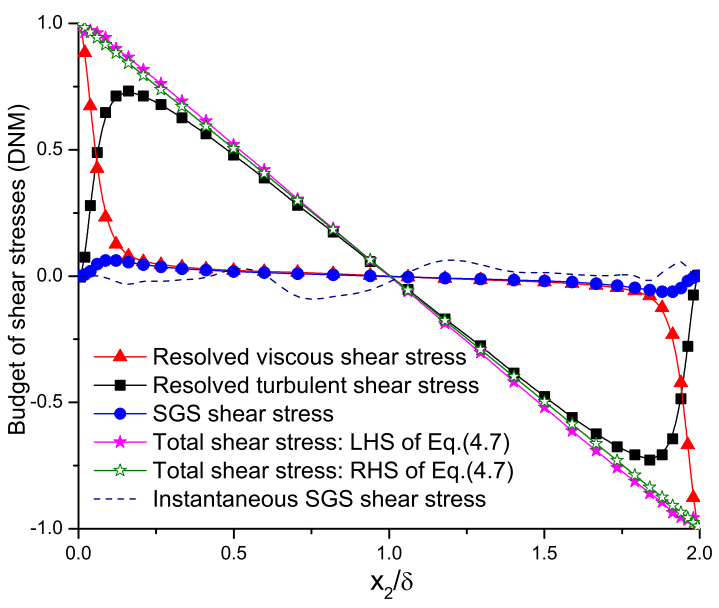

(a) DNM

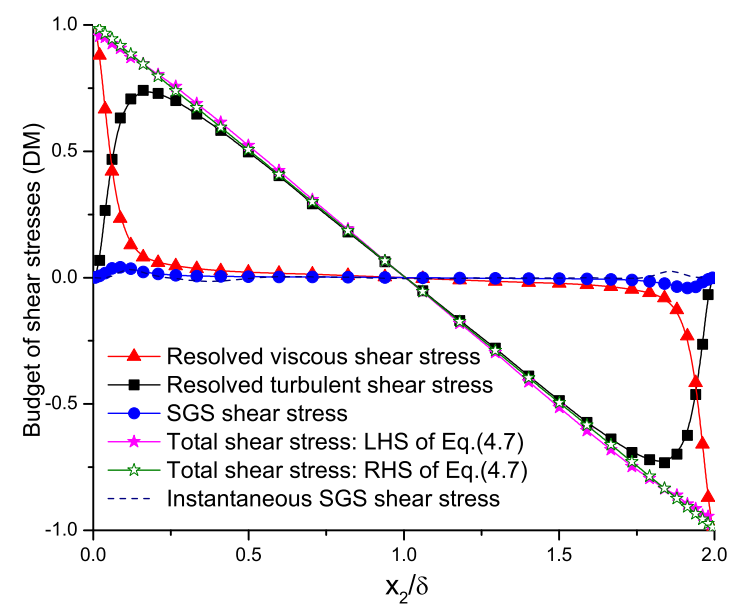

(b) $\mathrm{DM}$

FIGURE 4.8: Budget of shear stresses for $R o_{\tau}=15.0$ at $R e_{\tau}=150$ (nondimensionalized using $\tau_{w}^{a} / \rho$, predicted in conjunction with the DFLTDM). Location of the vertical line along which the instantaneous SGS shear stress component is obtained: $x_{1} / L_{1} \approx 0.5$ and $x_{3} / L_{3} \approx 0.5$.

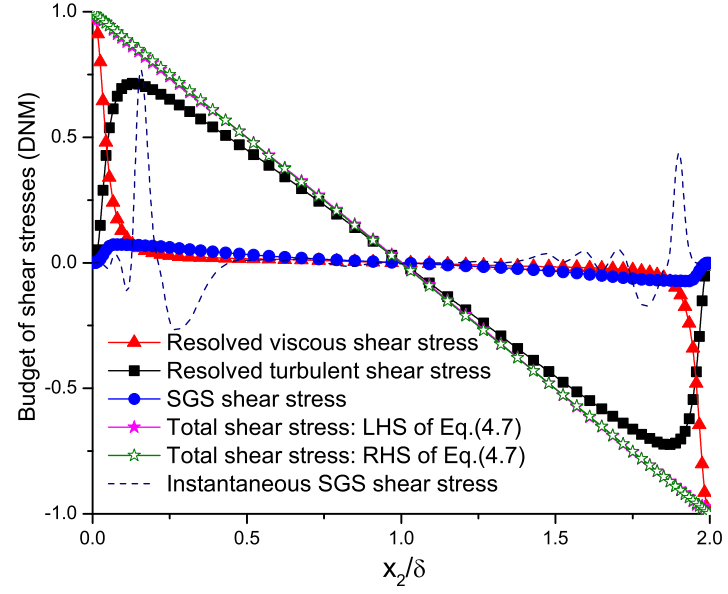

(a) DNM

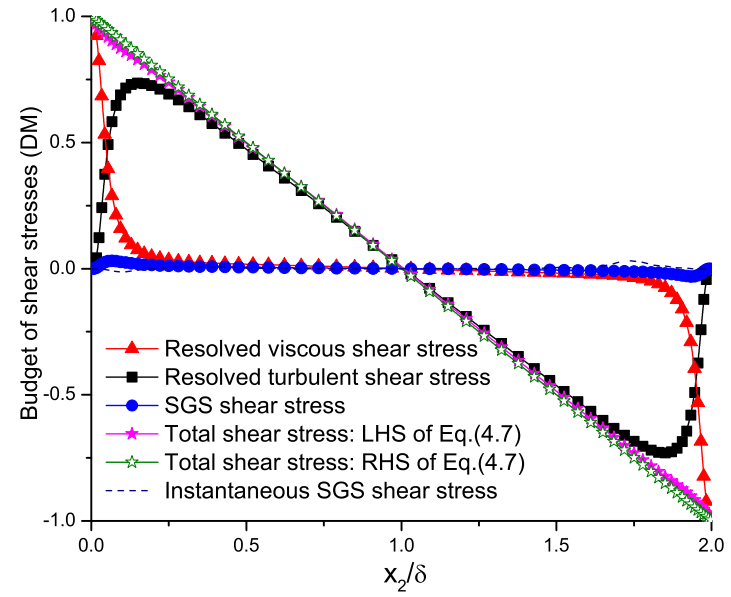

(b) $\mathrm{DM}$

FIGURE 4.9: Budget of shear stresses for $R o_{\tau}=2.5$ at $R e_{\tau}=300$ (nondimensionalized using $\tau_{w}^{a} / \rho$, predicted in conjunction with the DFLTDM). Location of the vertical line along which the instantaneous SGS shear stress component is obtained: $x_{1} / L_{1} \approx 0.5$ and $x_{3} / L_{3} \approx 0.5$.

$\left(\tau_{w h}=\left.\rho \nu \frac{\partial\left\langle\bar{u}_{1}\right\rangle}{\partial x_{2}}\right|_{x_{2}=0}\right)$, respectively.

Figures 4.8 and 4.9 show the shear stress budget predicted using the DNM and $\mathrm{DM}$ for $R e_{\tau}=150$ and 300, respectively. All quantities presented in these figures 


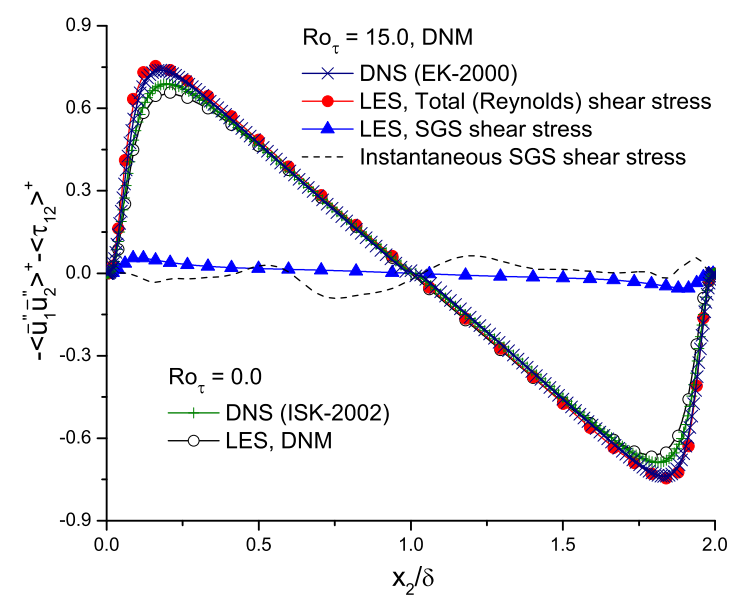

(a) DNM

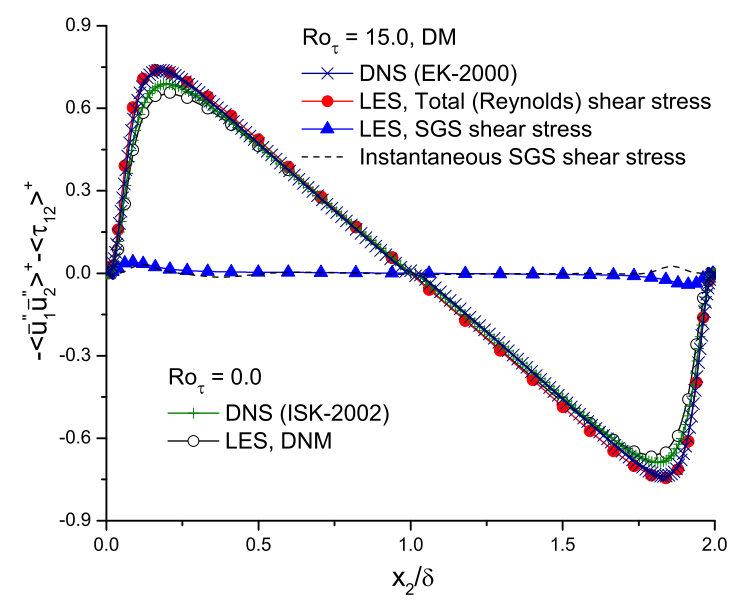

(b) $\mathrm{DM}$

FIGURE 4.10: Predicted Reynolds shear stress for $R o_{\tau}=0$ and 15 at $R e_{\tau}=150$ (nondimensionalized using $\tau_{w}^{a} / \rho$, predicted in conjunction with the DFLTDM). Location of the vertical line along which the instantaneous SGS shear stress component is obtained: $x_{1} / L_{1} \approx 0.5$ and $x_{3} / L_{3} \approx 0.5$.

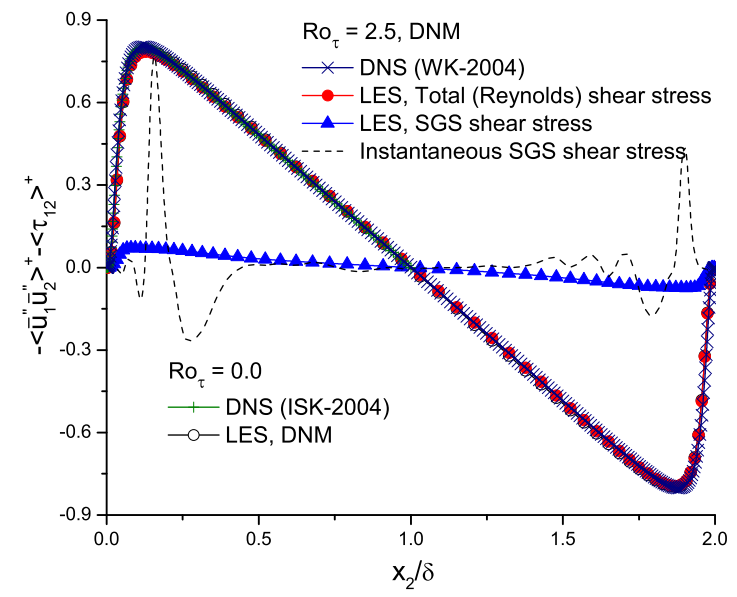

(a) DNM

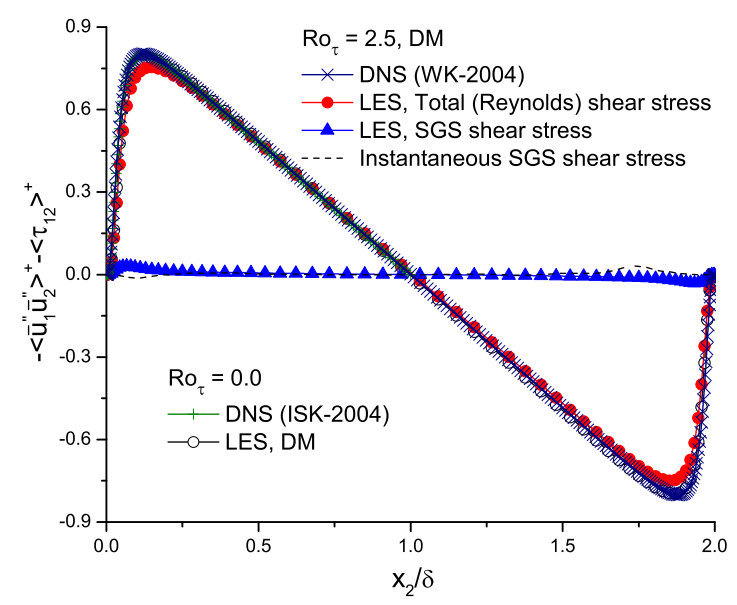

(b) $\mathrm{DM}$

FIGURE 4.11: Predicted Reynolds shear stress at $R o_{\tau}=0$ and 2.5 for $R e_{\tau}=300$ (non-dimensionalized using $\tau_{w}^{a} / \rho$, predicted in conjunction with the DFLTDM). Location of the vertical line along which the instantaneous SGS shear stress component is obtained: $x_{1} / L_{1} \approx 0.5$ and $x_{3} / L_{3} \approx 0.5$.

are non-dimensionalized using the viscous shear stress term averaged over the hot and cold walls (i.e., $\tau_{w}^{a} / \rho$ or $\left.\left(u_{\tau}^{a}\right)^{2}\right)$. It can be clearly seen from the figures that the total shear stress calculated from the LHS of Eq. (4.7) agrees very well with that calculated from the RHS of Eq. (4.7), implying that shear stress balance as expressed 
by Eq. (4.7) has been successfully reproduced by the numerical simulations based on the DNM and DM (and, this is in spite of the fact that two assumptions have been made in the derivation of this shear stress balance equation). As expected, the resolved viscous shear stress dominates in the viscous sublayer while the resolved turbulent shear stress dominates in the fully turbulent layer away from the solid wall. In general, the magnitude of $\left\langle-\tau_{12}\right\rangle$ is smaller than those of the other shear stress components. However, as shown in Figs. 4.8 and 4.9, the instantaneous value of the SGS shear stress $-\tau_{12}$ (shown using the dashed line) can be significant (even larger than the peak value of the resolved turbulence shear stress $\left.-\left\langle\bar{u}_{1}^{\prime \prime} \bar{u}_{2}^{\prime \prime}\right\rangle\right)$ at a specific location (e.g., at points along the vertical line determined by the intersection of the two planes at $x_{1} / L_{1}=0.5$ and $\left.x_{3} / L_{3}=0.5\right)$.

The time- and plane-averaged deviatoric part of the 'true' (or exact) Reynolds stresses (denoted using $-\left\langle u_{i}^{\prime e} u_{j}^{\prime e}\right\rangle$ ) obtained from a DNS approach can be estimated from LES using the resolved turbulent and SGS stresses [69,70], i.e. $-\left\langle u_{i}^{\prime e} u_{j}^{\prime e}\right\rangle=$ $-\left\langle\bar{u}_{i}^{\prime \prime} \bar{u}_{j}^{\prime \prime}\right\rangle-\left\langle\tau_{i j}\right\rangle$. Figures 4.10 and 4.11 compare the Reynolds shear stresses (i.e., $\left.-\left\langle\bar{u}_{1}^{\prime \prime} \bar{u}_{2}^{\prime \prime}\right\rangle^{+}-\left\langle\tau_{12}\right\rangle^{+}\right)$predicted using two SGS stress models with the estimated DNS results (i.e., $-\left\langle u_{1}^{\prime e} u_{2}^{\prime e}\right\rangle^{+}$). Quantities presented in these figures are non-dimensionalized using the averaged friction velocity $u_{\tau}^{a}$ over the hot and cold walls. By comparing Figs. 4.10(a) and (b), Figs. 4.11(a) and (b), it is observed that the total shear stresses obtained using the DNM and DM both confirm well with the DNS data both at $R e_{\tau}=150$ and 300. At $R e_{\tau}=150$, the mean SGS shear stress component $\left\langle\tau_{12}\right\rangle$ contributes a maximum of $8.74 \%$ and $8.08 \%$ to the total Reynolds shear stress $-\left\langle\bar{u}_{1}^{\prime \prime} \bar{u}_{2}^{\prime \prime}\right\rangle-\left\langle\tau_{12}\right\rangle$ for simulation based on the DNM and DM, respectively. At $R e_{\tau}=300$, it contributes a maximum of $8.43 \%$ and $3.45 \%$, respectively. This clearly indicates that the contribution from the SGS stress to the total Reynolds shear stress in the current LES cannot be neglected. Of course, the instantaneous contribution of the SGS shear stresses to the instantaneous Reynolds shear stresses can be much larger, which have been shown previously in Figs. 4.8 and 4.9. 


\subsubsection{Budget of Heat Fluxes}

Following a similar procedure used to derive Eq. (4.7) for the budget of the mean shear stresses, a budget equation for the time- and plane-averaged wall-normal heat fluxes at an arbitrary wall normal location $x_{2}$ can be obtained from the filtered energy equation (cf. Eq. (2.11)), viz.

$$
-\alpha \frac{\partial\langle\bar{\theta}\rangle}{\partial x_{2}}+\left\langle\bar{u}_{2}^{\prime \prime} \bar{\theta}^{\prime \prime}\right\rangle+\left\langle h_{2}\right\rangle=\frac{q_{w h}}{\rho c_{P}}
$$

where $q_{w h} \stackrel{\text { def }}{=}-\left.\lambda \frac{\partial\langle\bar{\theta}\rangle}{\partial x_{2}}\right|_{x_{2}=0}$ is the resolved molecular heat flux at the hot wall, and $\lambda$ is the thermal conductivity. The three terms on the LHS of Eq. (4.8) correspond to the resolved molecular heat flux, resolved turbulent heat flux and SGS heat flux, respectively.

All heat fluxes shown in Figs. 4.12 and 4.13 are non-dimensionalized using the molecular heat flux at the hot wall (viz., using $q_{w h} / \rho c_{P}=u_{\tau h} \theta_{\tau h}$ ). As shown in the figures, the total heat flux given by the RHS of Eq. (4.8) becomes unity after the normalization. In general, the total heat flux calculated from the LHS of Eq. (4.8) agrees with the theoretical value of 1.0. The heat transfer to or from the boundarylayer takes place by molecular conduction through the wall. Consequently, in the vicinity of the wall, the heat flux is entirely due to the resolved molecular heat flux. In the core region, the resolved turbulent heat flux dominates, accounting for the major contribution to the heat transfer in the wall-normal direction. As evident in Figs. 4.12 and 4.13, all these important physical features are well reproduced by LES.

Subfigures (a) and (b) of Figs. 4.12 and 4.13 compare the budget profiles for the SGS heat flux predicted using the DFLTDM model in conjunction with the two different SGS stress models (i.e., the DNM and DM). It is observed that the performance of the DFLTDM is similar in the context of two different SGS stress models. In LES of turbulent transport of a scalar, both the SGS stress model and the SGS scalar-flux model are important to the prediction of the filtered scalar field, because the filtered transport equations for the scalar and velocity fields are coupled. From 


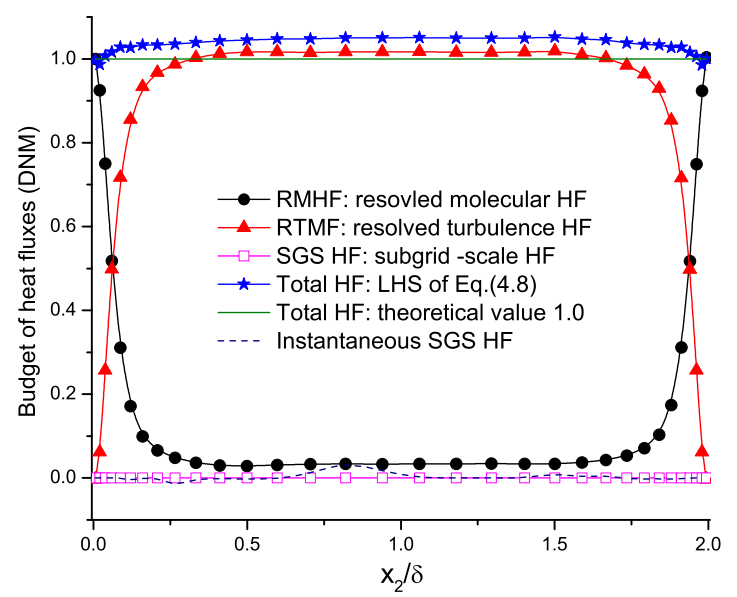

(a) DNM with DFLTDM

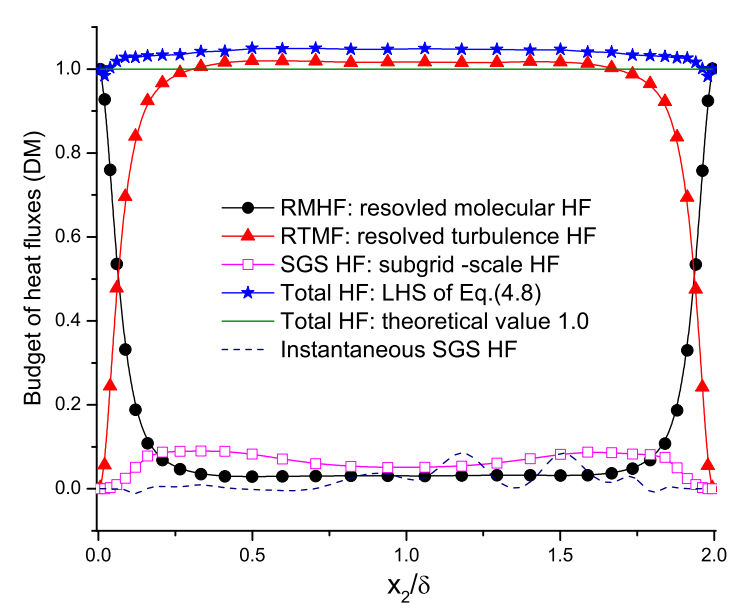

(b) DM with DFLTDM

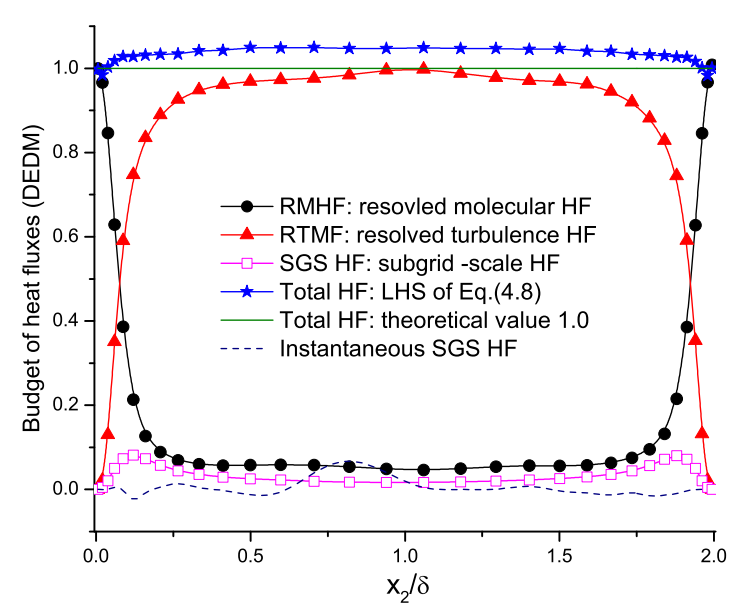

(c) DNM with DEDM

FIGURE 4.12: Budget of heat fluxes in the wall-normal direction for $R o_{\tau}=15.0$ at $R e_{\tau}=150$ (non-dimensionalized using $q_{w h} / \rho c_{P}$ ). Location of the vertical line along which the instantaneous SGS wall-normal HF component is obtained: $x_{1} / L_{1} \approx 0.5$ and $x_{3} / L_{3} \approx 0.5$.

Eq. (2.11), it is understood that at the subgrid scales, the SGS scalar-flux model has a more direct impact on the predicted filtered SGS scalar flux. To demonstrate, we compared the mean wall-normal heat flux budget predicted by the two different SGS HF models (i.e., the DFLTDM and DEDM) in conjunction with the same SGS stress model DNM. The results are displayed in Figs. 4.12(c) and 4.13(c). By comparing Figs. 4.12(a) and (c), and Fig. 4.13(a) and (c), it is seen that these two SGS HF models can result in significant differences in the prediction of the resolved turbulent and SGS wall-normal heat fluxes, even though the predictions of the total wall-normal 


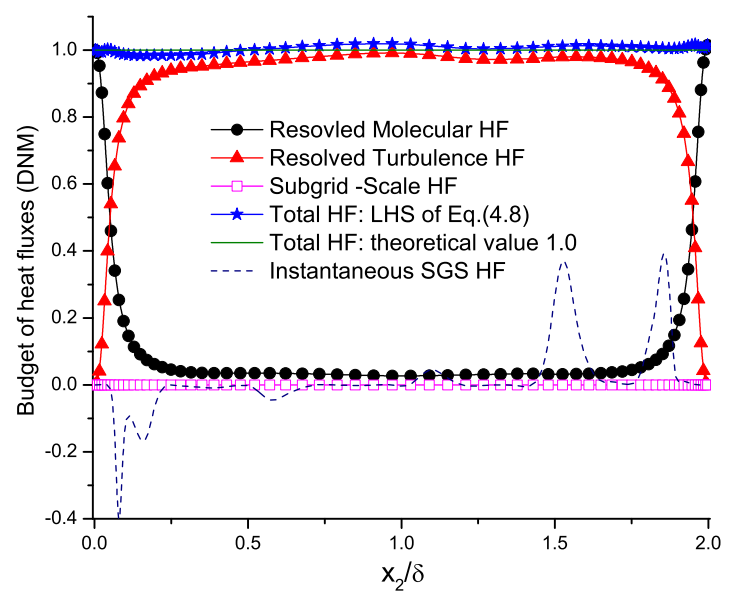

(a) DNM with DFLTDM

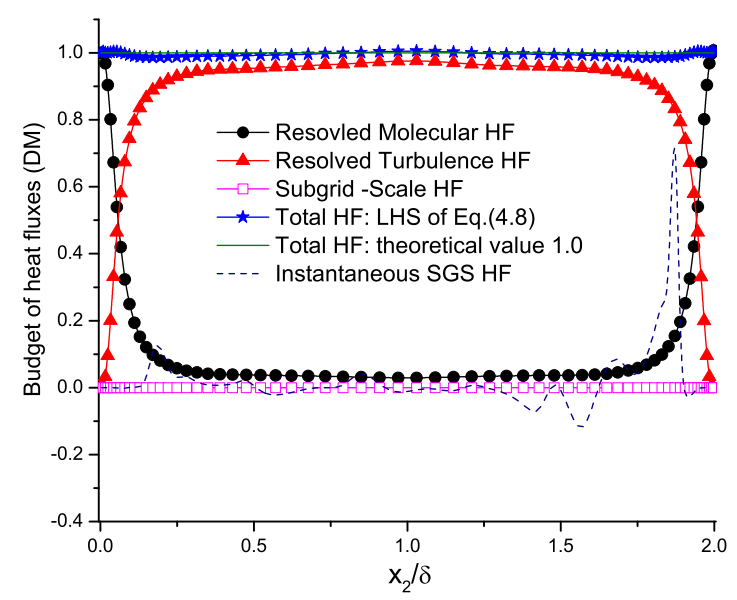

(b) DM with DFLTDM

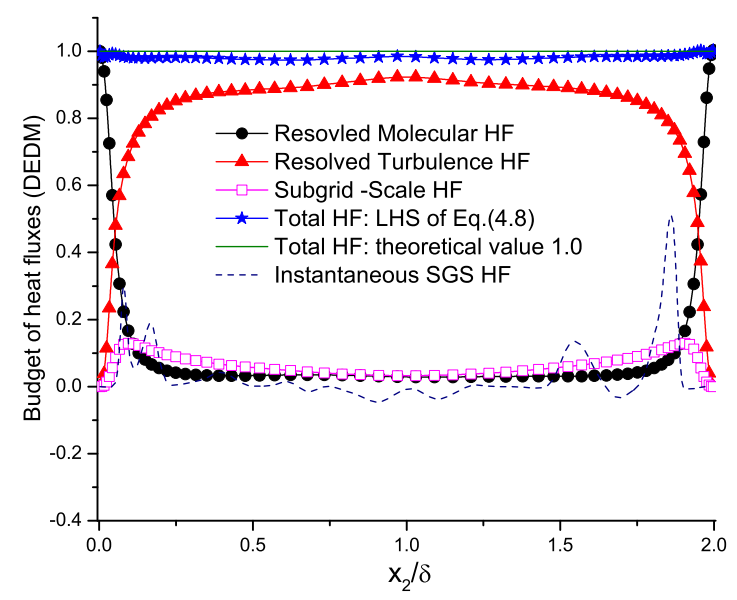

(c) DNM with DEDM

FIGURE 4.13: Budget of heat fluxes in the wall-normal direction for $R o_{\tau}=2.5$ at $R e_{\tau}=300$ (non-dimensionalized using $q_{w h} / \rho c_{P}$ ). Location of the vertical line along which the instantaneous SGS wall-normal HF component is obtained: $x_{1} / L_{1} \approx 0.5$ and $x_{3} / L_{3} \approx 0.5$.

heat flux are virtually identical (both approaching the theoretical value 1.0).

Similar to the Reynolds shear stresses, the time- and plane-averaged value of the 'true' (or exact) turbulent heat flux (denoted by $\left\langle u_{j}^{\prime e} \theta^{\prime e}\right\rangle$ ) obtained from a DNS approach can be estimated by LES using the resolved turbulent and SGS heat fluxes, i.e. $\left\langle u_{j}^{\prime e} \theta^{\prime e}\right\rangle=\left\langle\bar{u}_{j}^{\prime \prime} \bar{\theta}^{\prime \prime}\right\rangle+\left\langle h_{j}\right\rangle$. Figures 4.14 and 4.15 show the total streamwise, wallnormal and spanwise turbulent heat fluxes (i.e. $\left\langle\bar{u}_{1}^{\prime \prime} \bar{\theta}^{\prime \prime}\right\rangle^{+}+\left\langle h_{1}\right\rangle^{+},\left\langle\bar{u}_{2}^{\prime \prime} \bar{\theta}^{\prime \prime}\right\rangle^{+}+\left\langle h_{2}\right\rangle^{+}$ and $\left\langle\bar{u}_{3}^{\prime \prime} \bar{\theta}^{\prime \prime}\right\rangle^{+}+\left\langle h_{3}\right\rangle^{+}$) across the channel in comparison with the reported DNS data 


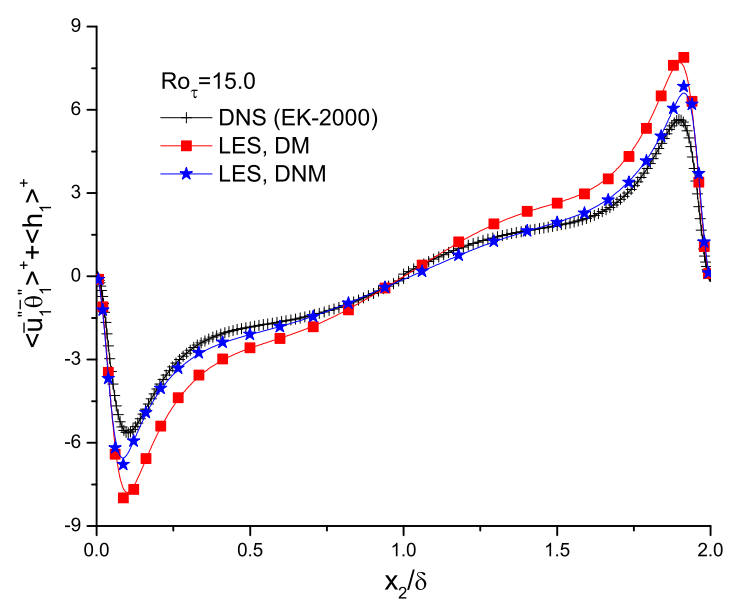

(a) Streamwise direction

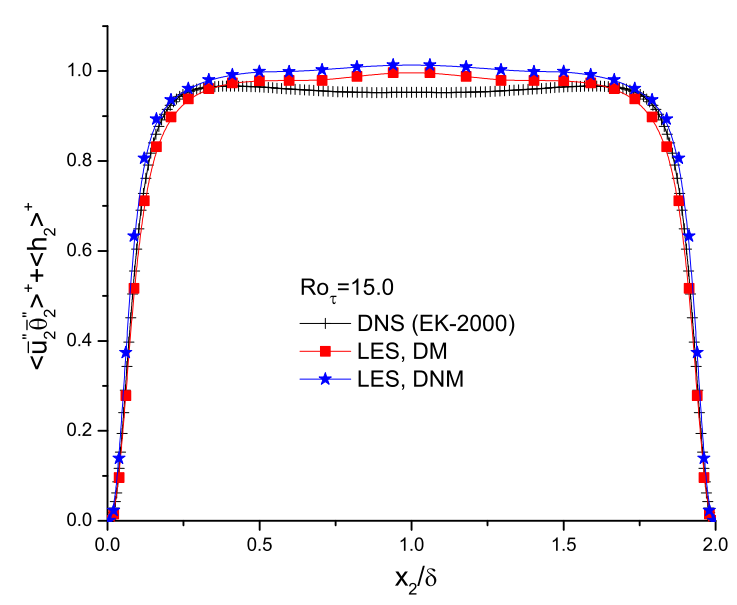

(b) Wall-normal direction

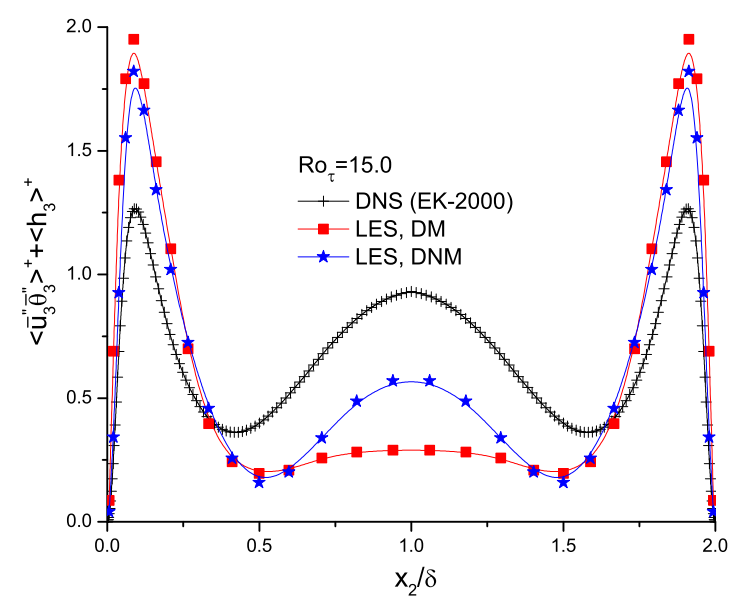

(c) Spanwise direction

FIGURE 4.14: Total turbulent heat fluxes at $R o_{\tau}=15.0$ for $R e_{\tau}=150$ (predicted in conjunction with the DFLTDM).

at $R e_{\tau}=150$ and 300, respectively. Quantities presented in these figures are nondimensionalized using the averaged friction velocity $u_{\tau}^{a}$ and friction temperature $\theta_{\tau}^{a}$ over the hot and cold walls. In general, the LES predictions of the wall-normal turbulent heat flux are in good agreement with the DNS data, but the turbulent heat fluxes are overpredicted by LES in the streamwise and spanwise directions. As shown in Figs. 4.14(a) and 4.15(a), in conjunction with the DFLTDM for modelling the SGS $\mathrm{HF}$, the DNM gives a slightly better performance than the DM. This indicates that it is beneficial to use a good SGS stress model for a more accurate calculation of the filtered flow field in a thermal-fluid coupled system, as this will subsequently improve 


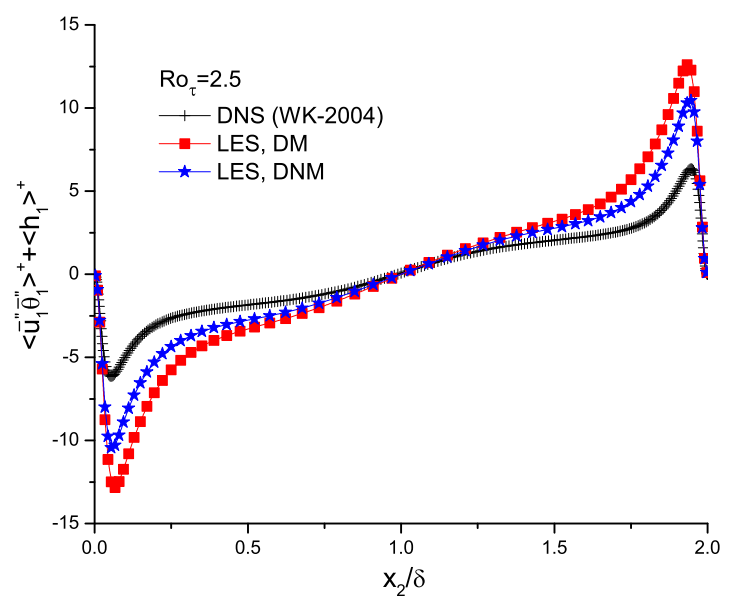

(a) Streamwise direction

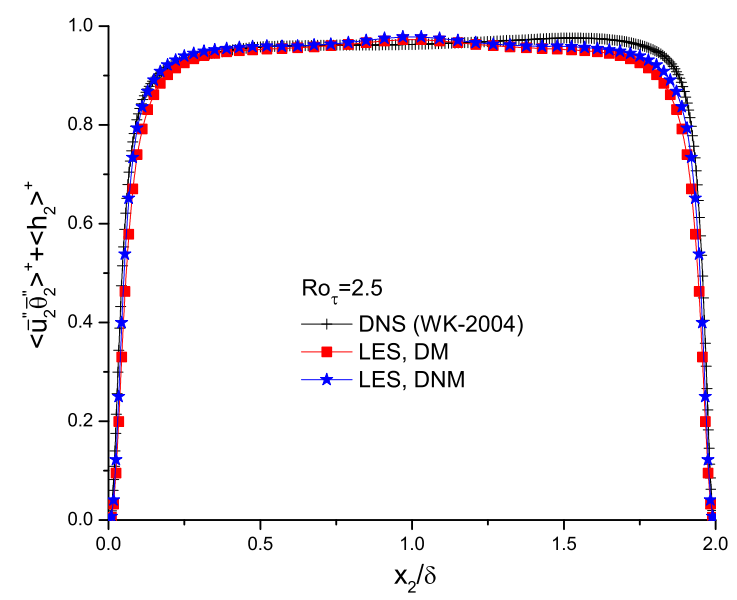

(b) Wall-normal direction

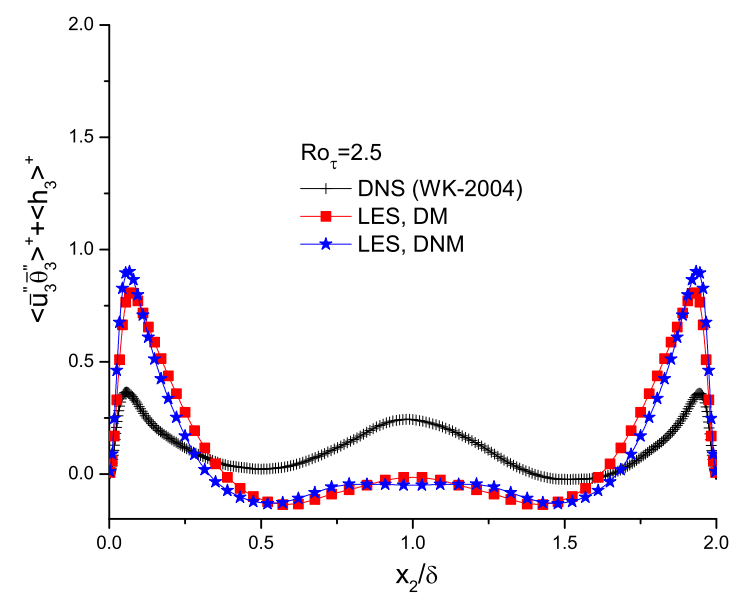

(c) Spanwise direction

FIGURE 4.15: Total turbulent heat fluxes at $R o_{\tau}=2.5$ for $R e_{\tau}=300$ (predicted in conjunction with the DFLTDM).

the prediction of the transport of the scalar (thermal energy).

Figures 4.16, 4.17 and 4.18 compare the profiles of the turbulent heat fluxes at different rotation numbers in streamwise, wall-normal and spanwise direction, respectively. At $R e_{\tau}=150$, it is shown in Fig. 4.16(a) that the streamwise turbulent heat flux $\left(\left\langle\bar{u}_{1}^{\prime \prime} \bar{\theta}^{\prime \prime}\right\rangle^{+}+\left\langle h_{1}\right\rangle^{+}\right)$decreases as the rotation number increases, and the location of the peak value shifts toward the wall. The other two turbulent heat fluxes in spanwise and wall-normal direction $\left(\left\langle\bar{u}_{2}^{\prime \prime} \bar{\theta}^{\prime \prime}\right\rangle^{+}+\left\langle h_{2}\right\rangle^{+}\right.$and $\left.\left\langle\bar{u}_{3}^{\prime \prime} \bar{\theta}^{\prime \prime}\right\rangle^{+}+\left\langle h_{3}\right\rangle^{+}\right)$, as shown in Figs. 4.17(a) and 4.18(a), increase with rotation numbers. By comparing subfigures 


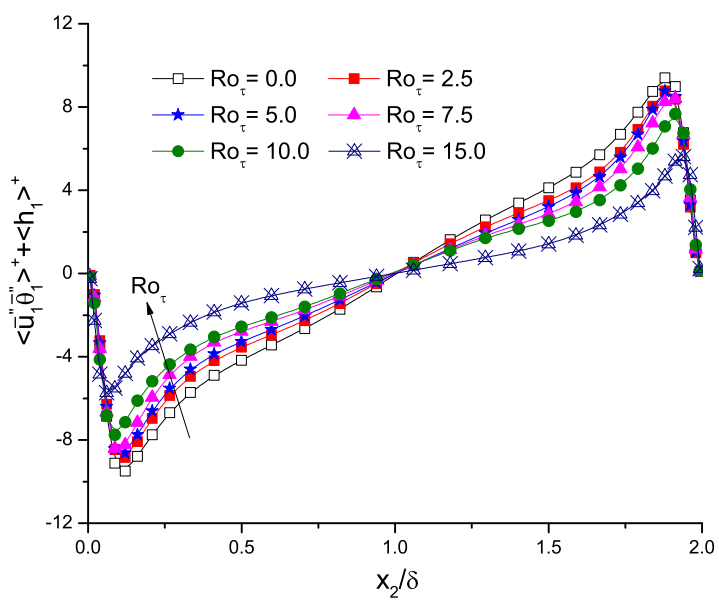

(a) $R e_{\tau}=150$

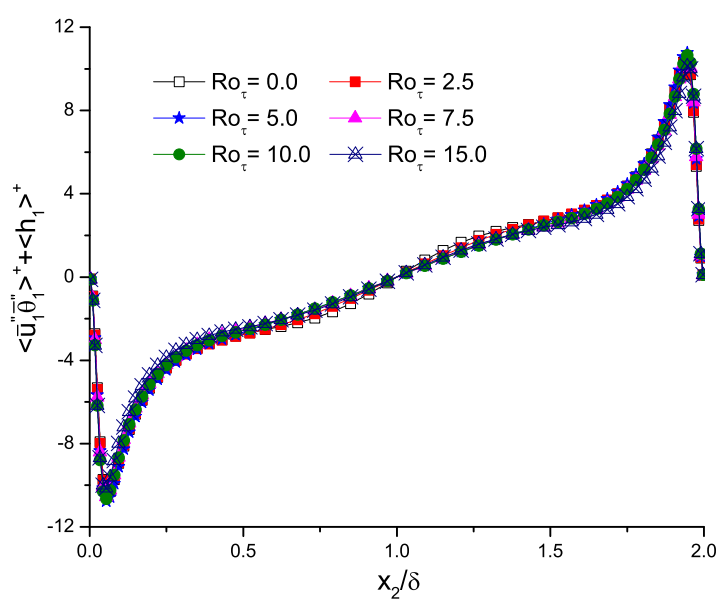

(b) $R e_{\tau}=300$

FIGURE 4.16: Total turbulent heat fluxes at various rotation number in streamwise direction (predicted in conjunction with the DFLTDM).

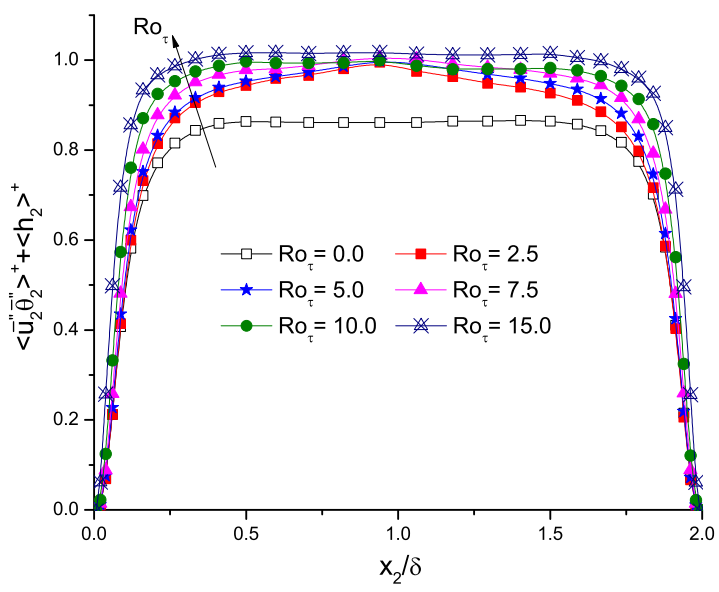

(a) $R e_{\tau}=150$

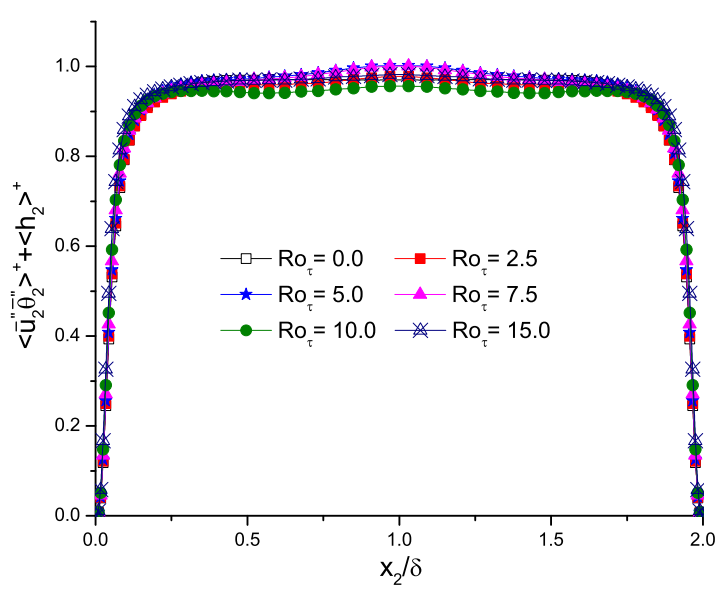

(b) $R e_{\tau}=300$

FIGURE 4.17: Total turbulent heat fluxes at various rotation number in wall-normal direction (predicted in conjunction with the DFLTDM).

(a) and (b) of Figs. 4.16, 4.17 and 4.18, it is observed that as the Reynolds number increases from $R e_{\tau}=150$ to 300, the turbulent heat flux levels in streamwise and wall-normal direction $\left(\left\langle\bar{u}_{1}^{\prime \prime} \bar{\theta}^{\prime \prime}\right\rangle^{+}+\left\langle h_{1}\right\rangle^{+}\right.$and $\left.\left\langle\bar{u}_{2}^{\prime \prime} \bar{\theta}^{\prime \prime}\right\rangle^{+}+\left\langle h_{2}\right\rangle^{+}\right)$are quite stable. However, the level of the heat flux in spanwise directoin $\left(\left\langle\bar{u}_{3}^{\prime \prime} \bar{\theta}^{\prime \prime}\right\rangle^{+}+\left\langle h_{3}\right\rangle^{+}\right)$increases noticeably. Furthermore, it is found that the heat fluxes are more sensitive to the system rotation at the lower Reynolds number. 


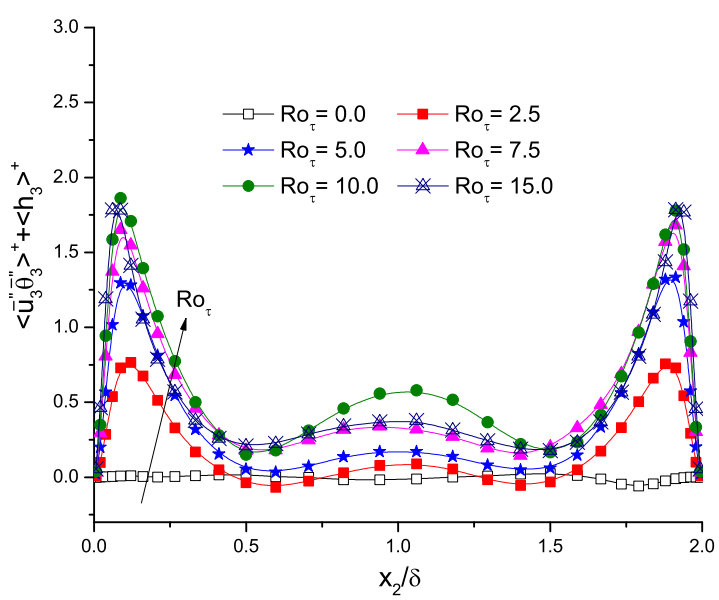

(a) $R e_{\tau}=150$

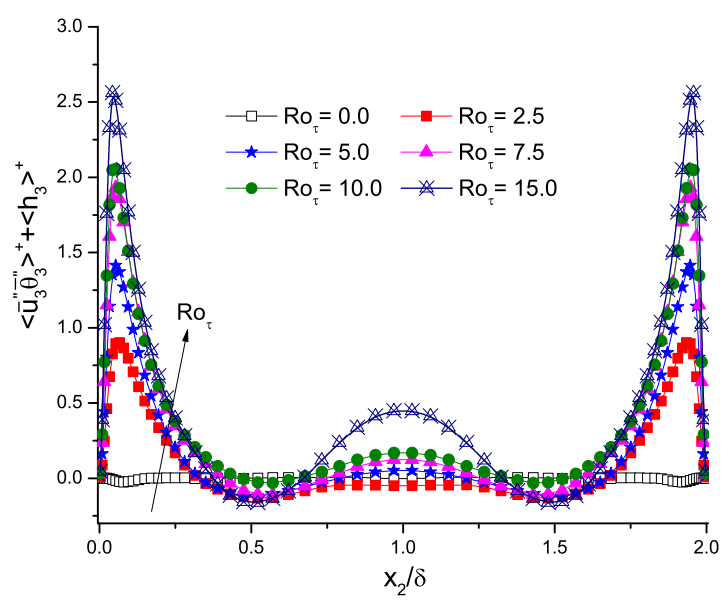

(b) $R e_{\tau}=300$

FIGURE 4.18: Total turbulent heat fluxes at various rotation number in spanwise direction (predicted in conjunction with the DFLTDM).

\subsection{Transfer of Local KE between the Resolved and Subgrid Scale Motions}

In the energy cascade of a turbulent flow, TKE is generated in the inertial subrange at the large scales of eddy motion and then transferred to and dissipated at the viscous scales. In LES, the viscous dissipative scales are not resolved. One of the most important functions of the SGS stress model is to 'drain' energy from the resolved scales to the unresolved scales, mimicking the actual energy dissipation. The transport of the resolved TKE is described by Eq. (A.12).

The SGS dissipation rate is defined as

$$
P_{r}=-\tau_{i j}^{*} \bar{S}_{i j}
$$

The SGS dissipation rate $P_{r}$ represents local KE transferred between the resolved and unresolved (subgrid) scales through an inertial and inviscid process. The instantaneous value of $P_{r}$ can be either positive or negative, representing a local forward or backward transfer of KE between the resolved and subgrid scales, respectively. It represents the rate of $\mathrm{KE}$ production and functions as a source of $\mathrm{KE}$ for the residual 
SGS motions and a sink of KE for the large resolved-scale motions (see, Eq. (A.12)). Figures 4.19 and 4.20 show the time- and plane-averaged profile of $P_{r}$ across the channel predicted using the DNM and DM, respectively. In both figures, the results are non-dimensionalized using $\left(u_{\tau}^{a}\right)^{4} / \nu$. The forward scatter (i.e., $\left.\left\langle P_{r}^{+}\right\rangle\right)$and backward scatter (i.e., $\left\langle P_{r}^{-}\right\rangle$) of the local KE have been separated, and these two quantities must verify: $\left\langle P_{r}\right\rangle=\left\langle P_{r}^{+}\right\rangle+\left\langle P_{r}^{-}\right\rangle$.

Substituting the constitutive relations for the DNM (represented by Eq. (2.30)) and DM (represented by Eq. (2.17)) into Eq. (4.9), then the equations for evaluating the SGS dissipation rate in the specific context of DNM and DM become

$$
P_{r}=C_{S} \beta_{i j} \bar{S}_{i j}+C_{W} \gamma_{i j} \bar{S}_{i j}+C_{N} \eta_{i j} \bar{S}_{i j}
$$

and

$$
P_{r}=2 C_{S} \bar{\Delta}^{2}|\bar{S}| \bar{S}_{i j} \bar{S}_{i j}=C_{S} \bar{\Delta}^{2}|\bar{S}|^{3}
$$

respectively.

As can be seen from Eq. (4.10), when the DNM is used, there are two factors determining the direction of the local KE flux between the resolved and subgrid scales of motions: one is the sign of the coefficients (i.e., $C_{S}, C_{W}$ and $C_{N}$ ), and the other is the relative geometric orientation between the filtered strain rate tensor $\bar{S}_{i j}$ and the constituent tensors $\beta_{i j}, \gamma_{i j}$ and $\eta_{i j}[11,71]$. In terms of physics, backscatter should not be simply viewed as a result of a negative SGS viscosity, but rather the non-alignment between the principal axes of $-\tau_{i j}$ and those of $\bar{S}_{i j}$ (i.e., the geometrical relationship between these two tensors $[11,13])$. As shown in Fig. 4.19, when the DNM is used for modelling the SGS stress tensor, the value of $P_{r}$ can be either positive and negative, implying that the physics associated with both forward and backward scatter of local KE between the resolved and unresolved (SGS) scales are captured.

It is well-known that the conventional DM of Lilly [7] is based on the overly simplistic linear Boussinesq assumption which requires that the principal axes of the negative SGS stress tensor $-\tau_{i j}$ be strictly aligned with those of the resolved strain 


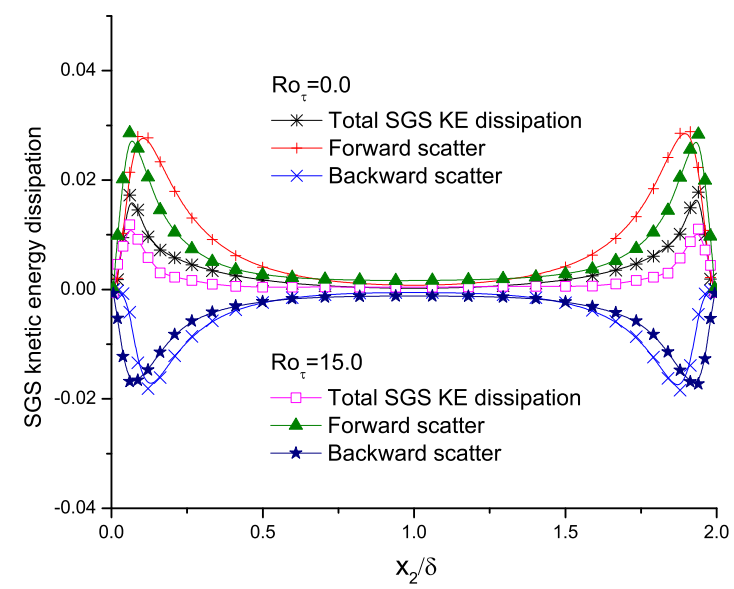

(a) $R e_{\tau}=150$

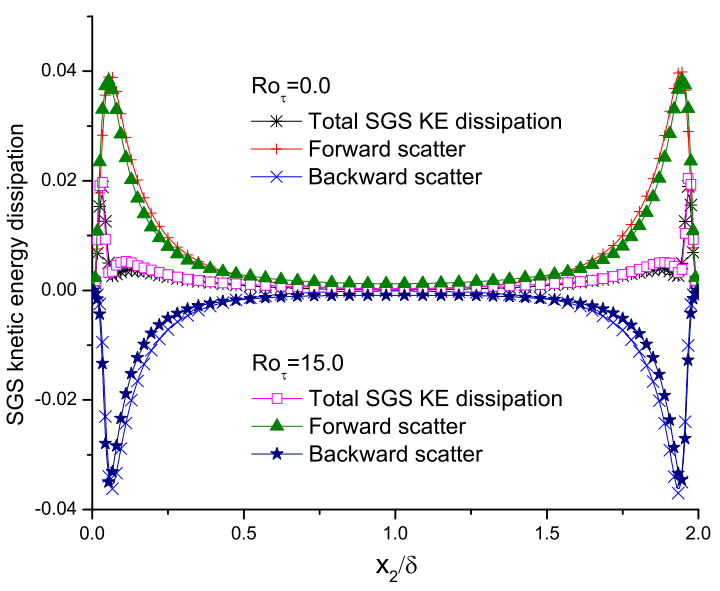

(b) $R e_{\tau}=300$

FIGURE 4.19: Time- and plane-averaged SGS KE dissipation rates (nondimensionalized using $\left(u_{\tau}^{a}\right)^{4} / \nu$, predicted using DNM in conjunction with the DFLTDM). Forward scatter: $\left\langle P_{r}^{+}\right\rangle$; backward scatter: $\left\langle P_{r}^{-}\right\rangle$; total SGS KE dissipation rate $\left\langle P_{r}\right\rangle$.

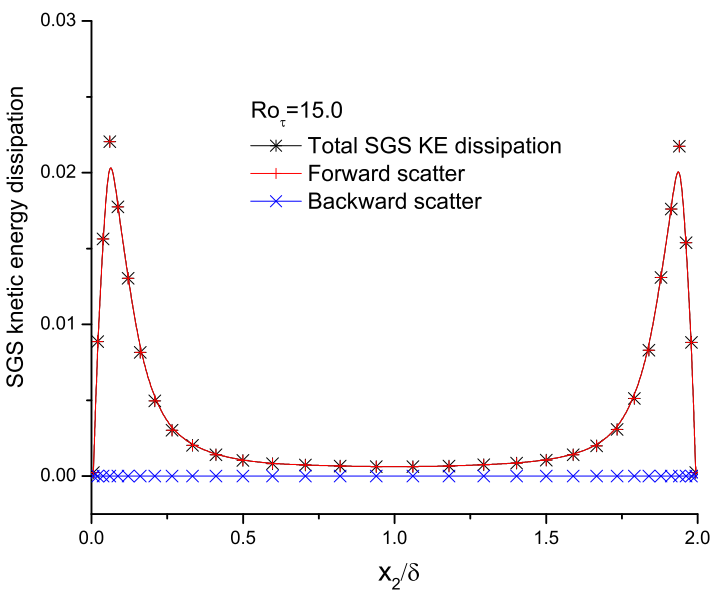

(a) $R e_{\tau}=150$

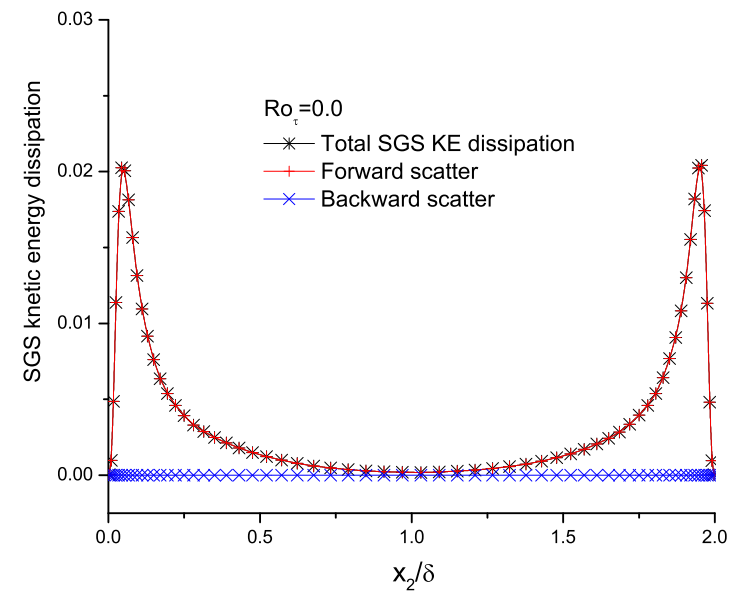

(b) $R e_{\tau}=300$

FIGURE 4.20: Time- and plane-averaged SGS KE dissipation rates (nondimensionalized using $\left(u_{\tau}^{a}\right)^{4} / \nu$, predicted using DM in conjunction with the DFLTDM). Forward scatter: $\left\langle P_{r}^{+}\right\rangle$; backward scatter: $\left\langle P_{r}^{-}\right\rangle$; total SGS KE dissipation rate $\left\langle P_{r}\right\rangle$.

rate tensor $\bar{S}_{i j}$. In consequence, for the DM, the sign of $P_{r}$ is determined entirely by the sign of model coefficient $C_{S}$. This is evident in Eq. (4.11)(since the value of $|\bar{S}|^{3}$ is always positive). This further implies that the KE transfer can only correspond to one of the following three scenarios: forward scatter if $C_{s}>0$, backward scatter if $C_{s}<0$, or no transport if $C_{s}=0$. As shown in Fig. 4.20, no net backscatter is 
observed over the period of time for which the flow statistics have been obtained, indicating that the DM has failed in reflecting the physics of local KE transfer from the unresolved to resolved scales in the context of the rotating flow tested.

By comparing Figs. 4.19(a) and (b), it is found that the peak values of both forward scatter and back scatter at $R e_{\tau}=300$ are larger than those at $R e_{\tau}=150$, and the locations of peaks are much closer to the wall. This is consistent with the fact that in a higher Reynolds number flow, the turbulence level is much higher and the boundary-layer where the viscous dissipation effect dominates becomes much thinner. Furthermore, by comparing the profiles of the non-rotating case and rotating case, it is found that the absolute magnitudes of both forward and backward scatter are slight lower for the rotating case, and the flow at a lower Reynolds number are more sensitive to the imposed system rotation. 


\section{Chapter 5}

\section{Conclusions and Reommendations for Future Work}

\section{$5.1 \quad$ Major Conclusions}

Two advanced SGS modelling approaches (namely, the DNM [11] for modelling the SGS stress tensor and the DFLTDM [9] for modelling the SGS heat flux vector) have been used to perform LES of a fully-developed heated channel flow subjected to streamwise system rotations. In order to conduct a comprehensive comparative study, two conventional SGS models were also tested in the simulations, including the DM of Lilly [72] for modelling the SGS stress tensor and the DEDM of Moin et al. [15] for modelling the SGS HF vector. This research focuses on examining the rotation effects on the turbulent flow field at different Reynolds numbers. To this purpose, the channel flow is charactrized by two Reynolds numbers $R e_{\tau}=150$ and 300, and a wide range of rotation numbers varying from $R o_{\tau}=0$ to 15 were tested for these two Reynolds numbers. The obtained LES results, in terms of the first- and second-order moments of statistics, have been thoroughly compared with the reported DNS data.

In response to the system rotation in the streamwise direction, two centrifugal forces and two Coriolis forces appear in the spanwise and wall-normal directions. The existence of the centrifugal and Coriolis forces makes the body-fixed coordinate system necessarily non-inertial and hence induces large secondary flow structures in the cross-stream $\left(x_{2}, x_{3}\right)$-planes. These large secondary flows in turn drastically 
alter the velocity field. The mean resolved spanwise velocity profile becomes skewsymmetric about the central wall-normal plane of the domain $\left(x_{2} / \delta=1\right)$, and four distinct layers of opposite motion co-exist between the two planes. The magnitude of the velocity of the secondary flow increases monotonically as the rotation number increases in the near-wall region.

This research also highlights a detailed analysis of the impact of streamwise system rotations on the transport of resolved turbulent stresses (i.e., $\left.\left\langle\bar{u}_{i}^{\prime \prime} \bar{u}_{j}^{\prime \prime}\right\rangle\right)$ and TKE (i.e., $\left\langle q^{2}\right\rangle$ ). The transport of $\left\langle\bar{u}_{i}^{\prime \prime} \bar{u}_{j}^{\prime \prime}\right\rangle$ and $\left\langle q^{2}\right\rangle$ are influenced jointly by two production terms, $P_{i j}$ and $G_{i j}$, related to the turbulent shear and rotation stresses, respectively. The production of the resolved TKE is not explicitly dependent upon the rotational angular speed $\Omega$, but rather, upon the resolved turbulent shear stresses and mean viscous shear stresses. As the rotation number increases, the mean resolved TKE (as well as all three mean resolved RMS velocities $\bar{u}_{1, r m s}^{+}, \bar{u}_{2, r m s}^{+}$and $\left.\bar{u}_{3, r m s}^{+}\right)$increases in the central core of the channel. In addition, by comparing the results of $R e_{\tau}=150$ and 300 , it can be concluded that the flow is less sensitive to the imposed system rotation at a higer Reynolds number. This is because at a given rotation number, the higher the Reynolds number, the larger the turbulent shear stresses (in comparison with the rotation stresses and Coriolis forces). In other words, at a given rotation number, the flow pattern becomes more towards Poiseuille type as the Reynolds number increases.

In order to study the predictive performance of the LES approach in terms of the momentum balance, the budget of the shear stresses across the channel has been examined. Numerical simulations based on the DNM and DM both have successfullly reproduced the shear stresses balance. Although the magnitude of the SGS shear stress component $\left\langle\tau_{i j}\right\rangle$ is small compared to the other shear stress components, its instantaneous value $\tau_{i j}$ can be very large and its contribution to the total Reynolds shear stress cannot be neglected. The DFLTDM model for SGS scalar heat flux in conjunction with two different SGS stress models (i.e., the DNM and DM) are used to predict the balance of heat fluxes. Numerical simulations show that different SGS HF models can result in significant differences in the prediction of the resolved turbulent and SGS wall-normal heat fluxes, even though the predictions of the total 
wall-normal heat flux are virtually identical. In conjunction with the DFLTDM for modelling the SGS HF, the DNM gives a slightly better performance than the DM in terms of the prediction of the streamwise turbulent heat flux. This indicates that it is beneficial to use a good SGS stress model for a more accurate calculation of the filtered flow field in a thermal-fluid coupled system, as this will subsequently improve the prediction of the transport of the scalar (thermal energy). As the Reynolds number increases from $R e_{\tau}=150$ to 300, the turbulent heat flux levels in streamwise and wall-normal direction $\left(\left\langle\bar{u}_{1}^{\prime \prime} \bar{\theta}^{\prime \prime}\right\rangle^{+}+\left\langle h_{1}\right\rangle^{+}\right.$and $\left.\left\langle\bar{u}_{2}^{\prime \prime} \bar{\theta}^{\prime \prime}\right\rangle^{+}+\left\langle h_{2}\right\rangle^{+}\right)$are quite stable. However, the level of the heat flux in spanwise directoin $\left(\left\langle\bar{u}_{3}^{\prime \prime} \bar{\theta}^{\prime \prime}\right\rangle^{+}+\left\langle h_{3}\right\rangle^{+}\right)$increases noticeably. Furthermore, it is found that the heat fluxes are more sensitive to the system rotation at a lower Reynolds number.

In terms of physics, forward and backward scatter of kinetic energy between the resolved and subgrid scales should not be simply viewed as a result of a negative SGS viscosity, but rather the non-alignment between the principal axes of $-\tau_{i j}$ and those of $\bar{S}_{i j}$. As a result of the simplicity in its constitutive relation (based as such on the linear Boussinesq assumption), the DM failed in reproducing the physical mechanism of backscatter. In contrast to the performance of the conventional DM of Lilly [7], the DNM is advantageous in reflecting the physical mechanism of both forward and backward scatter of kinetic energy between the resolved and subgrid scales. This salient property of the DNM is a result of the nonlinear constitutive relation adopted in its modeling formula, which allows for multiple degrees of freedom to determine the geometrical relation between the SGS stress tensor and the resolved strain and rotation rate tensors.

\subsection{Future Work}

Although the results of simulation and physical analysis are encouraging, in order to obtain a more comprehensive understanding of the coupled velocity and temperature fields and the predictive performance of the current LES approach, this research needs 
to be extended. In future studies, it would be required to include a comparative study of the current and other SGS models, and combination of a greater range of Reynolds numbers and rotation numbers. The present study provides a detailed analysis of the flow physics in a heated plane channel subjected to streamwise rotation. Turbulent flows in channels of different types of cross-sectional geometries (e.g., square and triangular ducts) subjected to arbitrary directional (e.g. streamwise and wall-normal) rotations also need to be thoroughly investigated in the future. 


\section{References}

[1] J. O. Hinze, Turbulence. New York: McGraw-Hill, 1975.

[2] U. Piomelli, Large-Eddy and Direct Simulation of Turbulent Flows, Lecture Notes for CFD2001. Kitchener, Ontario, May 27-29 2001. The 9th Annual Conference of the CFD Society of Canada.

[3] O. Reynolds, "An experimental investigation of the circumstances which determine whether the motion of water shall be direct or sinuous, and of the law of resistance in parallel channels," Philos. Trans. R. Soc. Lond. A, vol. 174, pp. 935-982, 1883.

[4] L. F. Richardson, "Weather prediction by numerical process," in Cambridge University Press, (Cambridge), 1922.

[5] A. N. Kolmogorov, "The local structure of turbulence in incompressible viscous fluid for very large reynolds numbers," in Proc. USSR Academy of Sciences, (Russian), pp. 299-303, 1941.

[6] J. Smagorinsky, "General circulation experiments with the primitive equations, I. the basic experiment," Mon. Weath. Rev., vol. 91, pp. 99-165, 1963.

[7] D. K. Lilly, "A proposed modification of the Germano subgrid-scale closure method," Phys. Fluids A, vol. 4, pp. 633-635, 1992. 
[8] J. H. Ferziger and M. Perić, Computational Methods for Fluid Dynamics. Berlin: Springer, 2nd ed., 1999.

[9] B.-C. Wang, E. Yee, D. J. Bergstrom, and O. Iida, "A new dynamic subgridscale heat-flux models for large eddy simulation of thermal convection based on the general gradient diffusion hypothesis," J. Fluid Mech., vol. 604, pp. 125-163, 2008.

[10] M. Germano, U. Piomelli, P. Moin, and W. H. Cabot, "A dynamic subgrid-scale eddy viscosity model," Phys. Fluids A, vol. 3, pp. 1760-1765, 1991.

[11] B.-C. Wang and D. J. Bergstrom, "A dynamic nonlinear subgrid-scale stress model," Phys. Fluids, vol. 17(035109), pp. 1-15, 2005.

[12] C. G. Speziale, "On nonlinear $k-l$ and $k-\varepsilon$ models of turbulence," J. Fluid Mech., vol. 178, pp. 459-475, 1987.

[13] B.-C. Wang, E. Yee, and D. J. Bergstrom, "Geometrical description of the subgrid-scale stress tensor based on Euler axis angle," AIAA J., vol. 44, pp. 11061110, 2006.

[14] Q.-Q. Xun, B.-C. Wang, and E. Yee, "Large-eddy simulation of turbulent heat convection in a spanwise rotating channel flow," Int. J. Heat Mass Trans., vol. 54, pp. 698-716, 2011.

[15] P. Moin, K. Squires, W. Cabot, and S. Lee, "A dynamic subgrid-scale model for compressible turbulence and scalar transport," Phys. Fluids A, vol. 3, pp. 27462757, 1991.

[16] M. V. Salvetti and S. Banerjee, "A priori tests of a new dynamic subgrid-scale 
model for finite-difference large-eddy simulations," Phys. Fluids, vol. 7, pp. 2831$2847,1995$.

[17] B.-C. Wang, E. Yee, D. J. Bergstrom, and O. Iida, "New dynamic subgrid-scale heat flux models for large-eddy simulation of thermal convection based on the general gradient diffusion hypothesis," J. Fluid Mech., vol. 604, pp. 125-163, 2008.

[18] J. Pallares and L. Davidson, "Large-eddy simulations of turbulent heat transfer in stationary and rotating square ducts," Phys. Fluids, vol. 14, pp. 2804-2816, 2002.

[19] R. V. R. Avancha and R. H. Pletcher, "Large eddy simulation of the turbulent flow past a backward-facing step with heat transfer and property variations," Int. J. Heat Fluid Flow, vol. 23, pp. 601-614, 2002.

[20] L. Wang and X.-Y. Lu, "An investigation of turbulent oscillatory heat transfer in channel flows by large eddy simulation," Int. J. Heat Mass Trans., vol. 47, pp. 2161-2172, 2004.

[21] B.-C. Wang, J. Yin, E. Yee, and D. Bergstrom, "A Complete and irreducible dynamic SGS heat-flux modelling based on the strain rate tensor for large-eddy simulation of thermal convection," Int. J. Heat Fluid Flow, vol. 28, pp. 1227$1243,2007$.

[22] S.-H. Peng and L. Davidson, "On a subgrid-scale heat flux model for large eddy simulation of turbulent thermal flow," Int. J. Heat Mass Trans., vol. 45, pp. 1393-1405, 2002.

[23] J. P. Johnston, R. M. Halleen, and D. K. Lezius, "Effects of spanwise rotation 
on the structure of two-dimensional fully developed turbulent channel flow," $J$. Fluid Mech., vol. 56, pp. 533-557, 1972.

[24] K. Nakabayashi and O. Kitoh, "Low reynolds number fully developed twodimensional turbulent channel flow with system rotation," J. Fluid Mech., vol. 315 , pp. 1-29, 1996.

[25] J. Kim, "The effect of rotation on turbulence structure," in Proc. Inter. Symp. Turbul. Shear Flow, (Karlsruhe, Germany), 1982.

[26] Y. Miyake and T. Kajishima, "Numerical simulation of the effects of Coriolis force on the structure of turbulence (1st report, global effects)," Bulletin of JSME, vol. 29, pp. 3341-3346, 1986.

[27] Y. Miyake and T. Kajishima, "Numerical simulation of the effects of Coriolis force on the structure of turbulence (2nd report, structure of turbulence)," Bulletin of JSME, vol. 29, pp. 3347-3351, 1986.

[28] U. Piomelli and J. Liu, "Large-eddy simulation of rotating channel flows using a localized dynamic model," Phys. Fluids, vol. 7, pp. 839-848, 1995.

[29] M. Tsubokura and N. Kobayashi, "Subgrid scale modeling for turbulence in rotating reference frames," J. Wind Eng. Ind. Aerodyn., vol. 81, pp. 361-375, 1999.

[30] D. P. Launder, B. E.and Tselspidakis and B. A. Younis, "A secondmoment closure study of rotating channel flow," J. Fluid Mech., vol. 183, pp. 63-75, 1987.

[31] Y. Shimomura, "A statistically derived two-equation model of turbulent shear flows in a rotating system," J. Phy. Soc. Jpn., vol. 58, pp. 352-355, 1989. 
[32] T. B. Gatski and C. G. Speziale, "On explicit algebraic stress models for complex turbulent flows," J. Fluid Mech., vol. 254, pp. 59-78, 1993.

[33] B. E. Launder and D. P. Tselepidakis, "Application of a new second-moment closure to turbulent channel flow rotating in orthogonal mode," Int. J. Heat Fluid Flow, vol. 15, pp. 2-10, 1994.

[34] Y. N. an H. Hattori, "An improved turbulence model for rotating shear flows," J. Turbulence, vol. 3, pp. 1-15, 2002.

[35] R. Kristoffersen and H. I. Andersson, "Direct simulations of low-Reynoldsnumber turbulent flow in a rotating channel," J. Fluid Mech., vol. 256, pp. 163$197,1993$.

[36] O. Elsamni and N. Kasagi, "The effects of system rotation with three orthogonal rotating axes on turbulent channel flow," in Proc. of "th International Congress on Fluid Dynamics and Propulsion, (Cairo, Egypt), 2001. CD-ROM.

[37] E. Lamballais, O. Metais, and M. Lesieur, "Spectral-dynamical model for largeeddy simulations of turbulent rotating channel flow," Theor. Comput. Fluid Dyn., vol. 12, pp. 149-177, 1998.

[38] O. Elsamni, Heat and momentum transfer in turbulent rotating channel flow. PhD thesis, Univ. of Tokyo, 2001.

[39] Q.-Q. Xun, B.-C. Wang, and E. Yee, "Quasi-Periodicity of the Drag Coefficient and Nusselt Number Induced by Taylor-Görtler Vortices," Numerical Heat Transfer: Part B, vol. 57, pp. 30-45, 2010.

[40] H. Wu and N. Kasagi, "Turbulent heat transfer in a channel flow with arbitrary 
directional system rotation," Int. J. Heat Mass Trans., vol. 47, pp. 4579-4591, 2004.

[41] B.-Y. Li, N.-S. Liu, and X.-Y. Lu, "Direct numerical simulation of wall-normal rotating turbulent channel flow with heat transfer," Int. J. Heat Mass Trans., vol. 49, pp. 1162-1175, 2006.

[42] A. Mehdizadeh, Direct numerical simulation, Lie Group analysis and modeiling of a turbulent channel flow with wall-normal rotation. PhD thesis, Technische University Darmstadt, 2009.

[43] M. Oberlack, W. Cabot, B. A. Pettersson-Reif, and T. Weller, "Group analysis, direct numerical simulation and modeling of turbulent channel flow with streamwise rotation," J. Fluid Mech., vol. 562, pp. 383-403, 2006.

[44] I. Recktenwald, C. Brücker, and W. Schröder, "Piv investigations of a turbulent channel flow rotating about the streamwise axis," in Proc. 10th European turbulence conference, (Trondheim, Norway), 2004.

[45] O. El-Samni and N. Kasagi, "On Turbulent Flow with Streamwise Rotation," Kyoto university research information repository, vol. 1141, pp. 128-136, 2000.

[46] T. Weller and M. Oberlack, "DNS of a Turbulent Channel Flow with Streamwise Rotation-Investigation on the Cross Flow Phenomena," Direct and Large-Eddy Simulation, vol. 6, pp. 241-248, 2006.

[47] I. Recktenwald, T. Weller, W. Schröder, and M. Oberlack, "Comparison of direct numerical simulations and particle-image velocimetry data of turbulent channel flow rotating about the streamwise axis," Phys. Fluids, vol. 19(085114), 2007. 
[48] N. Alkishriwi, M. Meinke, and W. Schröder, "Large-eddy simulation of streamwise-rotating turbulent channel flow," Comp. Fluids, vol. 37, pp. 786-792, 2008.

[49] F. F. Grinstein and C. Fureby, "Recent progress on MILES for high Reynolds number flows," ASME J. Fluids Eng., vol. 124, pp. 848-861, 2002.

[50] J. Pallares, F. X. Grau, and L. Davidson, "Pressure drop and heat transfer rates in forced convection rotating square duct flows at high rotation rates," Phys. Fluids, vol. 17 (075102), pp. 1-11, 2005.

[51] W.-W. Kim and S. Menon, "Application of the localized dynamic subgrid-scale model to turbulent wall-bounded flows," in Proc. 35th Aerospace Sciences Meeting $\mathscr{E}$ Exhibit, (Reno, Nevada), pp. 1-12, 1997. AIAA Paper 97-0210.

[52] M. Molla, B.-C. Wang, and D. C. S. Kuhn, "Large-eddy simulation of physilogical pulsatile flow based on a dynamic nonlinear subgrid-scale stress model," in Proc. ASME 9th Int. Conf. Nanochannels, Microchannels and Minichannels, (Edmonton, Canada), 2011. ICNMM2011-58052, 10 pages.

[53] H. Wu and N. Kasagi, "Test Case: Fully developed channel flow and heat transfer with streamwise system rotation at $R e_{\tau}=300$ and $R o_{\tau}=2.5$," http://www.thtlab.t.u-tokyo.ac.jp/, 2004.

[54] H. Wu and N. Kasagi, "Effects of arbitrary directional system rotation on turbulent channel flow," Phys. Fluids, vol. 16, pp. 979-990, 2004.

[55] S. B. Pope, Turbulent Flows. Cambridge, UK: Cambridge Univ., 2000.

[56] P. Sagaut and R. Grohens, "Discrete filters for large eddy simulation," Int. J. Numer. Meth. Fluids, vol. 31, pp. 1195-1220, 1999. 
[57] J. Pallares and L. Davidson, "Large-eddy simulations of turbulent flow in a rotating square duct," Phys. Fluids, vol. 12, pp. 2878-2894, 2000.

[58] Y. Zang, R. L. Street, and J. R. Koseff, "A dynamic mixed subgrid-scale model and its application to turbulent recirculating flows," Phys. Fluids A, vol. 5, pp. 3186-3196, 1993.

[59] U. Piomelli, "High Reynolds number calculations using the dynamic subgridscale stress model," Phys. Fluids A, vol. 5, pp. 1484-1490, 1993.

[60] B.-C. Wang, D. J. Bergstrom, J. Yin, and E. Yee, "Turbulence topologies predicted using large eddy simulations," J. Turbul., vol. 7, pp. 1-28, 2006.

[61] B.-C. Wang, J. Yin, E. Yee, and D. J. Bergstrom, "A complete and irreducible dynamic SGS heat-flux modelling based on the strain rate tensor for large-eddy simulation of thermal convection," Int. J. Heat Fluid Flow, vol. 28, pp. 1227$1243,2007$.

[62] J. Kim and P. Moin, "Application of a fractional-step method to incompressible Navier-Stokes equations," J. Comp. Phys., vol. 59, pp. 308-323, 1985.

[63] C. M. Rhie and W. L. Chow, "Numerical study of the turbulent flow past an airfoil with trailing edge separation," AIAA J., vol. 21, pp. 1525-1532, 1983.

[64] J. Yin, B.-C. Wang, and D. J. Bergstrom, "Geometrical properties of the resolved-scale velocity and temperature fields predicted using large-eddy simulation," Flow, Turbul. Combust., vol. 81, pp. 39-75, 2008.

[65] O. El-Samni and N. Kasagi, "Test Case: Fully Developed 2-D Channel Flow and Heat Transfer with Streamwise System Rotation at $R o_{\tau}=15$," http://www.thtlab.t.u-tokyo.ac.jp/, 2000. 
[66] A. Kuroda, N. Kasagi, and M. Hirata, "Direct numerical simulation of turbulent plane Couette-Poiseuille flows: Effect of mean shear rate on the near-wall turbulence structures," in Turbul. Shear Flows 9 (F. Durst, N. Kasagi, B. E. Launder, F. W. Schmidt, K. Suzuki, and J. H. Whitelaw, eds.), pp. 241-257, Berlin: Springer-Verlag, 1995.

[67] K. Iwamoto, Y. Suzuki, and N. Kasagi, "Test Case: Fully Developed 2-D Channel Flow at $R e_{\tau}=150, "$ http://www.thtlab.t.u-tokyo.ac.jp/, 2002.

[68] K. Iwamoto, Y. Suzuki, and N. Kasagi, "Test Case: Fully Developed 2-D Channel Flow at $R e_{\tau}=300, "$ http://www.thtlab.t.u-tokyo.ac.jp/, 2004.

[69] G. S. Winckelmans, H. Jeanmart, and D. Carati, "On the comparison of turbulence intensities from large-eddy simulation with those from experiment or direct numerical simulation," Phys. Fluids, vol. 14, pp. 1809-1811, 2002.

[70] P. Sagaut, Large Eddy Simulation for Incompressible Flows: An Introduction. Berlin: Springer, 3rd ed., 2006.

[71] B.-C. Wang, E. Yee, and D. J. Bergstrom, "Geometrical description of subgridscale stress tensor based on Euler axis/angle," AIAA J., vol. 44, pp. 1106-1110, 2006.

[72] D. K. Lilly, "The representation of small-scale turbulence in numerical simulation experiments," Proc. IBM Sci. Comp. Symp. on Eviron. Sci., p. 195, 1967. 


\section{Appendix A}

\section{Transport Equations for Resolved Turbulent Stresses}

In this appendix, the general transport equations for the resolved turbulent stresses and TKE in the context of LES of a turbulent flow subjected to a streamwise system rotation are derived. The mathematical approach follows the previous work of Xun et al. [14], who studied plane channel flows subjected to spanwise system rotations. The set of transport equations presented here hold also for a non-rotating flow, which can be obtained simply by dropping the terms related to the Coriolis forces.

Based on the Reynolds decomposition, an instantaneous filtered quantity can be expressed as:

$$
\bar{\phi}=[\bar{\phi}]+\bar{\phi}^{\prime \prime}
$$

where operator [.] represents ensemble-averaging and $\bar{\phi}^{\prime \prime}$ represents the residual component, with $\left[\bar{\phi}^{\prime \prime}\right] \equiv 0$. For the filtered velocity $\bar{u}_{i}$, the following relationships hold:

$$
\begin{gathered}
\bar{u}_{i} \bar{u}_{j}=\left[\bar{u}_{i}\right]\left[\bar{u}_{j}\right]+\left[\bar{u}_{i}\right] \bar{u}_{j}^{\prime \prime}+\left[\bar{u}_{j}\right] \bar{u}_{i}^{\prime \prime}+\bar{u}_{i}^{\prime \prime} \bar{u}_{j}^{\prime \prime}, \\
{\left[\bar{u}_{i} \bar{u}_{j}\right]=\left[\bar{u}_{i}\right]\left[\bar{u}_{j}\right]+\left[\bar{u}_{i}^{\prime \prime} \bar{u}_{j}^{\prime \prime}\right] .}
\end{gathered}
$$

By ensemble-averaging the filtered momentum equation (2.14), we obtain

$$
\frac{\partial\left[\bar{u}_{i}\right]}{\partial t}+\frac{\partial}{\partial x_{j}}\left(\left[\bar{u}_{i} \bar{u}_{j}\right]\right)=-\frac{1}{\rho} \frac{\partial[\bar{p}]}{\partial x_{i}}+\nu \frac{\partial^{2}\left[\bar{u}_{i}\right]}{\partial x_{j} \partial x_{j}}-\frac{\partial\left[\tau_{i j}\right]}{\partial x_{j}}+2 \varepsilon_{1 i j} \Omega\left[\bar{u}_{j}\right]
$$

Substituting Eqs. (A.2) and (A.3) into Eqs. (2.14) and (A.4), respectively, and then 
subtracting the resulting equations we get:

$$
\begin{aligned}
\frac{\partial \bar{u}_{i}^{\prime \prime}}{\partial t}+\left[\bar{u}_{j}\right] \frac{\partial \bar{u}_{i}^{\prime \prime}}{\partial x_{j}}= & -\bar{u}_{j}^{\prime \prime} \frac{\partial\left[\bar{u}_{i}\right]}{\partial x_{j}}-\frac{\partial}{\partial x_{j}}\left(\bar{u}_{i}^{\prime \prime} \bar{u}_{j}^{\prime \prime}-\left[\bar{u}_{i}^{\prime \prime} \bar{u}_{j}^{\prime \prime}\right]\right) \\
& -\frac{1}{\rho} \frac{\partial \bar{p}^{\prime \prime}}{\partial x_{i}}+\nu \frac{\partial^{2} \bar{u}_{i}^{\prime \prime}}{\partial x_{j} \partial x_{j}} \\
& -\frac{\partial\left(\tau_{i j}-\left[\tau_{i j}\right]\right)}{\partial x_{j}}+2 \varepsilon_{1 i j} \Omega \bar{u}_{j}^{\prime \prime} .
\end{aligned}
$$

Now multiply both sides of Eq. (A.5) by $\bar{u}_{k}^{\prime \prime}$ to give:

$$
\begin{aligned}
\bar{u}_{k}^{\prime \prime} \frac{\partial \bar{u}_{i}^{\prime \prime}}{\partial t}+\bar{u}_{k}^{\prime \prime}\left[\bar{u}_{j}\right] \frac{\partial \bar{u}_{i}^{\prime \prime}}{\partial x_{j}}= & -\bar{u}_{k}^{\prime \prime} \bar{u}_{j}^{\prime \prime} \frac{\partial\left[\bar{u}_{i}\right]}{\partial x_{j}}-\bar{u}_{k}^{\prime \prime} \frac{\partial}{\partial x_{j}}\left(\bar{u}_{i}^{\prime \prime} \bar{u}_{j}^{\prime \prime}-\left[\bar{u}_{i}^{\prime \prime} \bar{u}_{j}^{\prime \prime}\right]\right) \\
& -\frac{1}{\rho} \bar{u}_{k}^{\prime \prime} \frac{\partial \bar{p}^{\prime \prime}}{\partial x_{i}}+\nu \bar{u}_{k}^{\prime \prime} \frac{\partial^{2} \bar{u}_{i}^{\prime \prime}}{\partial x_{j} \partial x_{j}} \\
& -\bar{u}_{k}^{\prime \prime} \frac{\partial\left(\tau_{i j}-\left[\tau_{i j}\right]\right)}{\partial x_{j}}+2 \varepsilon_{1 i j} \Omega \bar{u}_{j}^{\prime \prime} \bar{u}_{k}^{\prime \prime} .
\end{aligned}
$$

Interchanging the indices $i$ and $k$ in Eq. (A.6) results in:

$$
\begin{aligned}
\bar{u}_{i}^{\prime \prime} \frac{\partial \bar{u}_{k}^{\prime \prime}}{\partial t}+\bar{u}_{i}^{\prime \prime}\left[\bar{u}_{j}\right] \frac{\partial \bar{u}_{k}^{\prime \prime}}{\partial x_{j}}= & -\bar{u}_{i}^{\prime \prime} \bar{u}_{j}^{\prime \prime} \frac{\partial\left[\bar{u}_{k}\right]}{\partial x_{j}}-\bar{u}_{i}^{\prime \prime} \frac{\partial}{\partial x_{j}}\left(\bar{u}_{k}^{\prime \prime} \bar{u}_{j}^{\prime \prime}-\left[\bar{u}_{k}^{\prime \prime} \bar{u}_{j}^{\prime \prime}\right]\right) \\
& -\frac{1}{\rho} \bar{u}_{i}^{\prime \prime} \frac{\partial \bar{p}^{\prime \prime}}{\partial x_{k}}+\nu \bar{u}_{i}^{\prime \prime} \frac{\partial^{2} \bar{u}_{k}^{\prime \prime}}{\partial x_{j} \partial x_{j}} \\
& -\bar{u}_{i}^{\prime \prime} \frac{\partial\left(\tau_{k j}-\left[\tau_{k j}\right]\right)}{\partial x_{j}}+2 \varepsilon_{1 k j} \Omega \bar{u}_{j}^{\prime \prime} \bar{u}_{i}^{\prime \prime} .
\end{aligned}
$$

Adding Eqs. (A.6) and (A.7) gives:

$$
\begin{aligned}
\frac{\partial\left(\bar{u}_{i}^{\prime \prime} \bar{u}_{k}^{\prime \prime}\right)}{\partial t}+\left[\bar{u}_{j}\right] \frac{\partial\left(\bar{u}_{i}^{\prime \prime} \bar{u}_{k}^{\prime \prime}\right)}{\partial x_{j}}= & -\bar{u}_{i}^{\prime \prime} \bar{u}_{j}^{\prime \prime} \frac{\partial\left[\bar{u}_{k}\right]}{\partial x_{j}}-\bar{u}_{k}^{\prime \prime} \bar{u}_{j}^{\prime \prime} \frac{\partial\left[\bar{u}_{i}\right]}{\partial x_{j}} \\
& +\bar{u}_{i}^{\prime \prime} \frac{\partial\left(\tau_{k j}-\left[\tau_{k j}\right]\right)}{\partial x_{j}}+\bar{u}_{k}^{\prime \prime} \frac{\partial\left(\tau_{i j}-\left[\tau_{i j}\right]\right)}{\partial x_{j}} \\
& \left.-\frac{\partial}{\partial x_{j}}\left(\bar{u}_{i}^{\prime \prime} \bar{u}_{j}^{\prime \prime} \bar{u}_{k}^{\prime \prime}\right)+\bar{u}_{k}^{\prime \prime} \frac{\partial}{\partial x_{j}}\left[\bar{u}_{i}^{\prime \prime} \bar{u}_{j}^{\prime \prime}\right]\right)+\bar{u}_{i}^{\prime \prime} \frac{\partial}{\partial x_{j}}\left[\bar{u}_{k}^{\prime \prime} \bar{u}_{j}^{\prime \prime}\right] \\
& -\frac{1}{\rho}\left(\bar{u}_{i}^{\prime \prime} \frac{\partial \bar{p}^{\prime \prime}}{\partial x_{k}}+\bar{u}_{k}^{\prime \prime} \frac{\partial \bar{p}^{\prime \prime}}{\partial x_{i}}\right)+\nu\left(\bar{u}_{i}^{\prime \prime} \frac{\partial^{2} \bar{u}_{k}^{\prime \prime}}{\partial x_{j} \partial x_{j}}+\bar{u}_{k}^{\prime \prime} \frac{\partial^{2} \bar{u}_{i}^{\prime \prime}}{\partial x_{j} \partial x_{j}}\right) \\
& +2 \varepsilon_{1 i j} \Omega \bar{u}_{j}^{\prime \prime} \bar{u}_{k}^{\prime \prime}+2 \varepsilon_{1 k j} \Omega \bar{u}_{j}^{\prime \prime} \bar{u}_{i}^{\prime \prime} .
\end{aligned}
$$

Applying the ensemble-averaging operation to Eq. (A.8), the following transport equa- 
tion for the resolved turbulent stress is obtained:

$$
\begin{aligned}
\underbrace{\frac{\partial\left[\bar{u}_{i}^{\prime \prime} \bar{u}_{k}^{\prime \prime}\right]}{\partial t}+\left[\bar{u}_{j}\right] \frac{\partial\left[\bar{u}_{i}^{\prime \prime} \bar{u}_{k}^{\prime \prime}\right]}{\partial x_{j}}}_{\mathrm{I}}= & \underbrace{-\left[\bar{u}_{i}^{\prime \prime} \bar{u}_{j}^{\prime \prime}\right] \frac{\partial\left[\bar{u}_{k}\right]}{\partial x_{j}}-\left[\bar{u}_{k}^{\prime \prime} \bar{u}_{j}^{\prime \prime}\right] \frac{\partial\left[\bar{u}_{i}\right]}{\partial x_{j}}}_{\mathrm{II}} \\
& \underbrace{-\left(\left[\tau_{i j} \frac{\left.\partial \bar{u}_{k}^{\prime \prime}\right]}{\left.\partial x_{j}\right]}+\left[\tau_{k j} \frac{\left.\partial \bar{u}_{i}^{\prime \prime}\right]}{\left.\partial x_{j}\right]}\right)\right.\right.}_{\mathrm{III}}+\underbrace{\frac{\partial}{\partial x_{j}}\left(\left[\bar{u}_{i}^{\prime \prime} \tau_{k j}\right]+\left[\bar{u}_{k}^{\prime \prime} \tau_{i j}\right]\right)}_{\mathrm{IV}} \\
& -\underbrace{\frac{\partial}{\partial x_{j}}\left[\bar{u}_{i}^{\prime \prime} \bar{u}_{j}^{\prime \prime} \bar{u}_{k}^{\prime \prime}\right]}_{\mathrm{V}}-\underbrace{\frac{1}{\rho}\left(\left[\bar{u}_{i}^{\prime \prime} \frac{\partial \bar{p}^{\prime \prime}}{\partial x_{k}}\right]+\left[\bar{u}_{k}^{\prime \prime} \frac{\partial \bar{p}^{\prime \prime}}{\partial x_{i}}\right]\right)}_{\mathrm{VI}} \\
& +\underbrace{\nu\left(\left[\bar{u}_{i}^{\prime \prime} \frac{\partial^{2} \bar{u}_{k}^{\prime \prime}}{\partial x_{j} \partial x_{j}}\right]+\left[\bar{u}_{k}^{\prime \prime} \frac{\partial^{2} \bar{u}_{i}^{\prime \prime}}{\partial x_{j} \partial x_{j}}\right]\right)}_{\mathrm{VIII}}
\end{aligned}
$$

The role of each of term in this equation can be identified as follows:

(I) represents the local rate of change and advection by the mean flow of the resolved turbulent shear stress;

(II) represents the production term due to the action of the resolved turbulent stresses on the gradient of the mean velocity, and describes the interaction between the mean and turbulent parts of the flow;

(III) represents the production term due to the action of the SGS stresses on the gradient of the residual velocity, and is associated with the interaction between the SGS stress and velocity fluctuations;

(IV) is the SGS diffusion term;

$(\mathrm{V})$ is the triple correlation for the fluctuating flow field related to turbulent advection; (VI) is the velocity-pressure-gradient tensor;

(VII) is the viscous diffusion and dissipation term;

(VIII) represents the production term due to rotation effects.

Equation (A.9) represents the general transport equation for the resolved turbulent shear stress $\left[\bar{u}_{i}^{\prime \prime} \bar{u}_{k}^{\prime \prime}\right]$. For a plane channel flow, statistics based on time- and plane-averaged quantities (denoted using $\langle\cdot\rangle$ ) are of more interest to the researcher, 
because the flow can be further assumed to be: (1) statistically stationary, and (2) homogeneous in the $\left(x_{1}, x_{3}\right)$-plane. For LES of a channel flow, an instantaneous filtered quantity can be decomposed into a time- and plane-averaged component and a residual component (i.e., $\bar{\phi}=\langle\bar{\phi}\rangle+\bar{\phi}^{\prime \prime}$ ). The procedure to derive the transport equation for the turbulent stress $\left\langle\bar{u}_{i}^{\prime \prime} \bar{u}_{k}^{\prime \prime}\right\rangle$ is identical to the procedure used in the derivation of $\left[\bar{u}_{i}^{\prime \prime} \bar{u}_{k}^{\prime \prime}\right]$. However, with these two additional assumptions for a fully-developed plane channel flow, the transport equation for $\left\langle\bar{u}_{i}^{\prime \prime} \bar{u}_{k}^{\prime \prime}\right\rangle$ can be further simplified to give:

$$
\begin{aligned}
\frac{D\left\langle\bar{u}_{i}^{\prime \prime} \bar{u}_{k}^{\prime \prime}\right\rangle}{D t}=0= & -\left\langle\bar{u}_{i}^{\prime \prime} \bar{u}_{2}^{\prime \prime}\right\rangle \frac{\partial\left\langle\bar{u}_{k}\right\rangle}{\partial x_{2}}-\left\langle\bar{u}_{k}^{\prime \prime} \bar{u}_{2}^{\prime \prime}\right\rangle \frac{\partial\left\langle\bar{u}_{i}\right\rangle}{\partial x_{2}} \\
& -\left(\left\langle\tau_{i j} \frac{\partial \bar{u}_{k}^{\prime \prime}}{\partial x_{j}}\right\rangle+\left\langle\tau_{k j} \frac{\partial \bar{u}_{i}^{\prime \prime}}{\partial x_{j}}\right\rangle\right)+\frac{\partial}{\partial x_{2}}\left(\left\langle\bar{u}_{i}^{\prime \prime} \tau_{k 2}\right\rangle+\left\langle\bar{u}_{k}^{\prime \prime} \tau_{i 2}\right\rangle\right) \\
& -\frac{\partial}{\partial x_{2}}\left\langle\bar{u}_{i}^{\prime \prime} \bar{u}_{2}^{\prime \prime} \bar{u}_{k}^{\prime \prime}\right\rangle-\frac{1}{\rho}\left(\left\langle\bar{u}_{i}^{\prime \prime} \frac{\partial \bar{p}^{\prime \prime}}{\partial x_{k}}\right\rangle+\left\langle\bar{u}_{k}^{\prime \prime} \frac{\partial \bar{p}^{\prime \prime}}{\partial x_{i}}\right\rangle\right) \\
& +\nu\left(\left\langle\bar{u}_{i}^{\prime \prime} \frac{\partial^{2} \bar{u}_{k}^{\prime \prime}}{\partial x_{j} \partial x_{j}}\right\rangle+\left\langle\bar{u}_{k}^{\prime \prime} \frac{\partial^{2} \bar{u}_{i}^{\prime \prime}}{\partial x_{j} \partial x_{j}}\right\rangle\right) \\
& +2 \Omega\left(\varepsilon_{1 i j}\left\langle\bar{u}_{j}^{\prime \prime} \bar{u}_{k}^{\prime \prime}\right\rangle+\varepsilon_{1 k j}\left\langle\bar{u}_{j}^{\prime \prime} \bar{u}_{i}^{\prime \prime}\right\rangle\right)
\end{aligned}
$$

where $D(\cdot) / D t \stackrel{\text { def }}{=} \partial(\cdot) / \partial t+\left\langle\bar{u}_{j}\right\rangle \cdot \partial(\cdot) / \partial x_{j}$ is the material derivative. In particular, the transport equation for $\left\langle\bar{u}_{1}^{\prime \prime} \bar{u}_{2}^{\prime \prime}\right\rangle$ can be derived from Eq. (A.10) as

$$
\begin{aligned}
\frac{D\left\langle\bar{u}_{1}^{\prime \prime} \bar{u}_{2}^{\prime \prime}\right\rangle}{D t}=0= & -\left\langle\bar{u}_{2}^{\prime \prime 2}\right\rangle \frac{\partial\left\langle\bar{u}_{1}\right\rangle}{\partial x_{2}} \\
& -\left(\left\langle\tau_{1 j} \frac{\partial \bar{u}_{2}^{\prime \prime}}{\partial x_{j}}\right\rangle+\left\langle\tau_{2 j} \frac{\partial \bar{u}_{1}^{\prime \prime}}{\partial x_{j}}\right\rangle\right)+\frac{\partial}{\partial x_{2}}\left(\left\langle\bar{u}_{1}^{\prime \prime} \tau_{22}\right\rangle+\left\langle\bar{u}_{2}^{\prime \prime} \tau_{12}\right\rangle\right) \\
& -\frac{\partial}{\partial x_{2}}\left\langle\bar{u}_{1}^{\prime \prime} \bar{u}_{2}^{\prime \prime 2}\right\rangle-\frac{1}{\rho}\left(\left\langle\bar{u}_{1}^{\prime \prime} \frac{\partial \bar{p}^{\prime \prime}}{\partial x_{2}}\right\rangle+\left\langle\bar{u}_{2}^{\prime \prime} \frac{\partial \bar{p}^{\prime \prime}}{\partial x_{1}}\right\rangle\right) \\
& +\nu\left(\left\langle\bar{u}_{1}^{\prime \prime} \frac{\partial^{2} \bar{u}_{2}^{\prime \prime}}{\partial x_{j} \partial x_{j}}\right\rangle+\left\langle\bar{u}_{2}^{\prime \prime} \frac{\partial^{2} \bar{u}_{1}^{\prime \prime}}{\partial x_{j} \partial x_{j}}\right\rangle\right) \\
& +2 \Omega\left(\left\langle\bar{u}_{1}^{\prime \prime} \bar{u}_{3}^{\prime \prime}\right\rangle\right) .
\end{aligned}
$$

Similarly, the transport equation for the resolved turbulent normal stress $\left\langle\bar{u}_{k}^{\prime \prime} \bar{u}_{k}^{\prime \prime}\right\rangle$ (no 
summation implied here) can be shown to take the following form:

$$
\begin{aligned}
\frac{D\left\langle\bar{u}_{k}^{\prime \prime} \bar{u}_{k}^{\prime \prime}\right\rangle}{D t}=0= & -2\left\langle\bar{u}_{k}^{\prime \prime} \bar{u}_{2}^{\prime \prime}\right\rangle \frac{\partial\left\langle\bar{u}_{k}\right\rangle}{\partial x_{2}} \\
& -2\left\langle\tau_{k j} \frac{\partial \bar{u}_{k}^{\prime \prime}}{\partial x_{j}}\right\rangle+2 \frac{\partial\left\langle\bar{u}_{k}^{\prime \prime} \tau_{k 2}\right\rangle}{\partial x_{2}} \\
& -\frac{\partial}{\partial x_{2}}\left\langle\bar{u}_{2}^{\prime \prime} \bar{u}_{k}^{\prime \prime 2}\right\rangle-\frac{2}{\rho}\left\langle\bar{u}_{k}^{\prime \prime} \frac{\partial \bar{p}^{\prime \prime}}{\partial x_{k}}\right\rangle \\
& +2 \nu\left\langle\bar{u}_{k}^{\prime \prime} \frac{\partial^{2} \bar{u}_{k}^{\prime \prime}}{\partial x_{j} \partial x_{j}}\right\rangle+4 \Omega \varepsilon_{1 k j}\left\langle\bar{u}_{j}^{\prime \prime} \bar{u}_{k}^{\prime \prime}\right\rangle .
\end{aligned}
$$

